\title{
Unification of parton and coupled-wire approaches to quantum magnetism in two dimensions
}

\author{
Eyal Leviatan (ㄱ) and David F. Mross $\odot$ \\ Department of Condensed Matter Physics, Weizmann Institute of Science, Rehovot 7610001, Israel
}

\begin{abstract}
(Received 16 July 2020; revised 20 October 2020; accepted 2 December 2020; published 29 December 2020)
\end{abstract}
\begin{abstract}
The fractionalization of microscopic degrees of freedom is a remarkable manifestation of strong interactions in quantum many-body systems. Analytical studies of this phenomenon are primarily based on two distinct frameworks: field theories of partons and emergent gauge fields, or coupled arrays of one-dimensional quantum wires. We unify these approaches for two-dimensional spin systems. Via exact manipulations, we demonstrate how parton gauge theories arise in microscopic wire arrays and explicitly relate spin operators to emergent quasiparticles and gauge-field monopoles. This correspondence allows us to compute physical correlation functions within both formulations and leads to a straightforward algorithm for constructing parent Hamiltonians for a wide range of exotic phases. We exemplify this technique for several chiral and nonchiral quantum spin liquids.
\end{abstract}

DOI: 10.1103/PhysRevResearch.2.043437

\section{INTRODUCTION}

Determining the ground state of interacting spin systems is a quintessential problem in quantum condensed matter physics. Generic lattice Hamiltonians that are solely restricted by symmetries and locality typically yield ground states that spontaneously break one or more microscopic symmetries. Moreover, these phases exhibit only short-range entanglement and are therefore considered conventional. The tendency toward triviality can be avoided when additional ingredients, such as geometric frustration, prevent the formation of classical order and instead promote so-called quantum spin liquid (QSL) ground states [1-5]. These phases of matter are not characterized by any local order parameter. Instead, their principal feature is the existence of low-energy excitations that carry fractional quantum numbers and/or exhibit fractional statistics. In the cases of gapped QSLs, this definition can be sharpened into the notion of topological order [6], which manifests itself in a universal nonlocal contribution to the ground-state entanglement [7-9] and a ground-state degeneracy on nontrivial manifolds.

Various solvable models have unambiguously demonstrated the possibility of QSL ground states [10-14]. However, the exact solution relies on rather fine-tuned interactions. In more generic models, strong evidence of QSL ground states has been found numerically using exact diagonalization [15,16], quantum Monte Carlo [17-19], variational Monte Carlo [20-25], and density matrix renormalization group [26-33]. Finally, several recent experiments provide tantalizing evidence for QSLs. Notable examples include $\kappa-\mathrm{ET}_{2}$ [34-38], Pd(dmit $)_{2}$ [38-41], herbertsmithite [42-44], and $\alpha-$ $\mathrm{RuCl}_{3}[45,46]$.

Published by the American Physical Society under the terms of the Creative Commons Attribution 4.0 International license. Further distribution of this work must maintain attribution to the author $(s)$ and the published article's title, journal citation, and DOI.
A major theoretical challenge toward studying QSL candidate Hamiltonians is the intrinsic nonlocality of their fractional excitations. Well-known examples of such quasiparticles are spinons, neutral spin- $\frac{1}{2}$ excitation that may be bosons or fermions. In conventional magnets, two such spinons can only appear together and form spin-1 magnons. By contrast, individual spinons become liberated in QSLs. Both the nonlocality of spinons and their ability to appear in isolation can be encoded via an emergent gauge field under which they are charged. Individual spinons are not gauge invariant and thus not directly accessible to any (local) probe. Still, they may constitute bona fide quasiparticles when the gauge field is in a deconfined phase.

The primary workhorse for analytically describing such phases is known as parton construction (see, e.g., Refs. $[47,48]$ ). Its starting point is a representation of lattice spins in terms of parton creation operators $\psi_{\vec{r}, \sigma}^{\dagger}$, e.g., $S_{\vec{r}}^{+}=$ $\psi_{\vec{r}, \uparrow}^{\dagger} \psi_{\vec{r}, \downarrow}$. In a constrained Hilbert space with exactly one parton per site, these operators can be used to faithfully represent any microscopic spin Hamiltonian. However, the main utility of this representation is the ability to determine possible longwavelength theories that may, as a matter of principle, emerge from a lattice model with certain microscopic input, such as symmetries. The parton construction amounts to temporarily ignoring the constraint, which permits new mean-field $A n$ sätze that are highly nontrivial in terms of microscopic spins. Refining the mean-field theory to include fluctuations reveals the expected gauge structure. A key feature of this approach is its versatility in capturing various gapped and gapless QSLs as well as conventional phases. Its main drawback is an intrinsic difficulty to relate a given QSL phase to a specific spin model. The most tangible connection between parton constructions and microscopic Hamiltonians is through projected wave functions [49]. However, such analyses are biased by the choice of mean field and are computationally expensive.

Over the past years, an alternative technique that shines precisely at this Achilles' heel of parton approaches has gained in popularity. In "coupled-wire constructions," 
microscopic parent Hamiltonians for strongly correlated phases are constructed explicitly. Additionally, creation operators of fractional quasiparticles are expressible in terms of microscopic degrees of freedom. This approach was pioneered by Kane, Mukhopadhyay, and Lubensky for fractional quantum Hall states [50]. Recently, it has been extended to capture many other topological or strongly correlated phases, including (i) a wider range of fractional quantum Hall [51-59] and quantum spin Hall [60] states, (ii) Chern insulators [61-63] and superconductors [64-69], (iii) exotic surface states of symmetry-protected topological phases [70,71], (iv) correlated states in twisted bilayer graphene [72], (v) three-dimensional topological orders [73-78], (vi) Weyl semimetals [79,80], and (vii) several QSLs [81-90]. Furthermore, coupled-wire methods have been used to construct anyon models [91,92], classify symmetry-protected topological phases [93,94], and derive field-theoretic dualities in $2+1$ dimensions $[95,96]$. For an introduction and overview of this approach, see also Ref. [97].

A natural starting point for applying this technique to QSLs is given by a spin system where all couplings in the $\hat{y}$ direction, say, have been switched off. The resulting model may be profitably viewed as an array of one-dimensional spin chains (i.e., wires) along the $\hat{x}$ direction. Each spin chain is then taken to form a gapless one-dimensional QSL. The corresponding long-wavelength degrees of freedom form a coarse-grained basis for reintroducing interwire couplings. When these interactions are strongly relevant in the renormalization group sense, they may drive the system into a bona fide two-dimensional phase. Unfortunately, no general principle for the construction of parent Hamiltonians within this framework is known. Instead, it has to be done on a laborious case-by-case basis. Consequently, only a limited number of QSLs have been accessed in this manner [81-90].

We present a framework that unifies the two approaches based on the well-known particle-vortex duality of bosons in $2+1$ dimensions [98-100]. Its recent implementation in the coupled-wire formalism [96] allows us to transcribe fieldtheoretic insights into explicit models and thereby achieve the desired connection between parton and coupled-wire methods. We find remarkably simple relationships between microscopic degrees of freedom, such as the Néel vector $\vec{N}_{y}(x)$ or the valence-bond operator $\epsilon_{y}(x)$ and parton operators in a suitable gauge. For example, we show that

$$
\begin{gathered}
\sum_{\tilde{y}} f_{\tilde{y}, \sigma, \chi_{\sigma}}^{\dagger} f_{\tilde{y}-2, \sigma, \bar{x}_{\sigma}}+\text { H.c. }=\sum_{y} N_{y+1}^{+} N_{y}^{-}+\text {H.c., } \\
\sum_{\tilde{y}} f_{\tilde{y}, \sigma, R}^{\dagger} f_{\tilde{y}, \sigma, L}+\text { H.c. }=\sum_{y}\left[\epsilon_{y+1} \epsilon_{y}-N_{y+1}^{z} N_{y}^{z}\right],
\end{gathered}
$$

where $f_{\tilde{y}, \sigma, \chi}^{\dagger}$ creates a fermionic parton with chirality $\chi=$ $R / L$ and spin $\sigma=\uparrow / \downarrow$ on the "dual" wire $\tilde{y}=y+1 / 2$. In the first equation, $\chi_{\sigma}=R(L)$ for $\sigma=\uparrow(\downarrow)$ and $\bar{\chi}_{\sigma}=L(R)$ is the opposite chirality. These relations are represented graphically in Fig. 1.

Crucially, any coupled-wire model that separately conserves the two parton species maps onto a local spin model. Parent spin Hamiltonians for a wide range of nontrivial phases can thus be generated by constructing weakly correlated twodimensional band insulators or superconductors of partons.
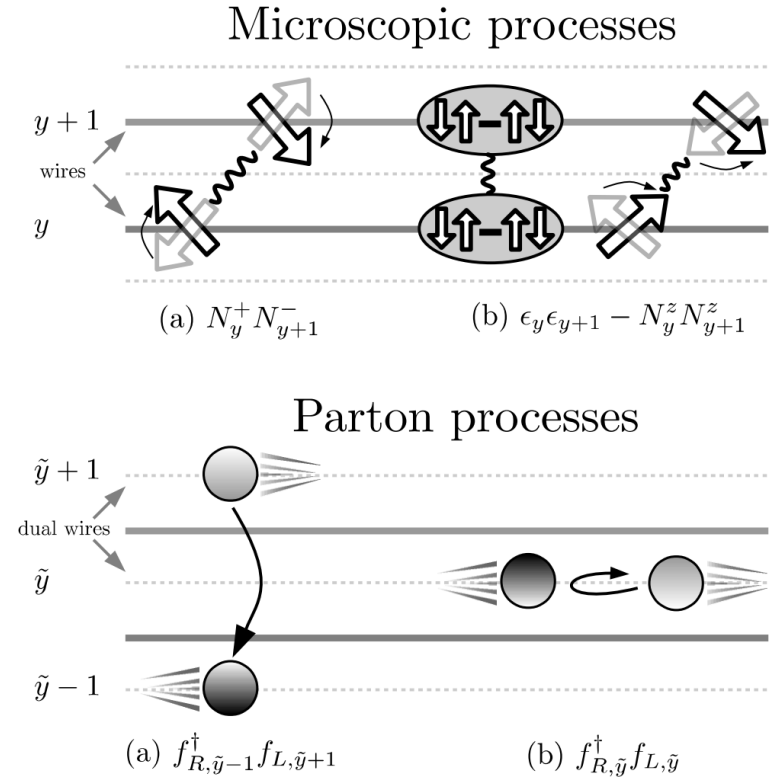

FIG. 1. Microscopic interwire processes and the fermionicparton counterparts onto which they map: (a) spin exchange between neighboring wires translates to fermion hopping by two dual wires, (b) the indicated combination of dimer-dimer and Ising interactions translates to fermion umklapp scattering on the corresponding dual wire.

An example of such a model is shown in Fig. 2, which illustrates the spin Hamiltonian for a $\mathbb{Z}_{2}$ QSL obtained from a superconductor of fermionic partons. Moreover, coupled-wire models for various strongly correlated states of bosons and fermions are also known in the literature [51,52,54-57,5964,66-71]. Each of these corresponds to a local spin model as well. These models realize QSLs that are not describable by a parton mean-field Ansatz but require a further fractionalization of the partons.

In addition to constructing parent Hamiltonians for specific gapped phases, we use the exact transformation between spins and partons to derive the gauge theory for the latter. We explicitly relate monopoles in the emergent gauge field to spin operators and determine the action of microscopic

Decoupled spin chains Coupled spin-chain array

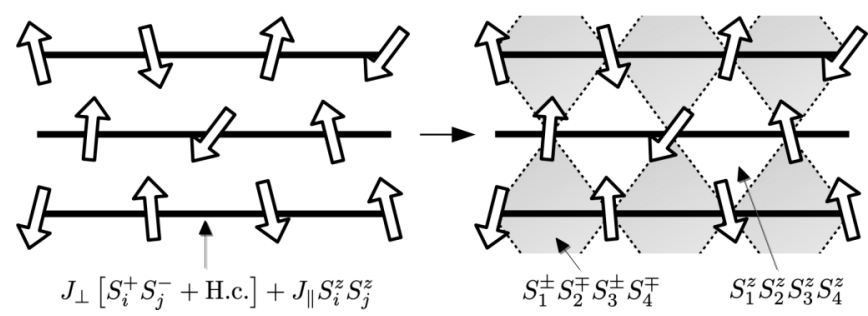

FIG. 2. The parent Hamiltonian of a $\mathbb{Z}_{2}$ spin liquid can be obtained by translating the coupled-wire model that realizes a BCS superconductor of fermionic partons. The gray and white diamonds represent the indicated interaction between spins at their corners. These terms are reminiscent of those realizing Kitaev's toric code [10]. However, the present model conserves $S^{z}$ and realizes a phase with dynamical matter fields. See Sec. V B 5 for the derivation and a detailed discussion. 
symmetries. These properties demonstrate the desired unification of partons and coupled wires. They, moreover, indicate that the same approach may be extended to gapless states in the future.

The rest of this paper is organized as follows: In Sec. II, we briefly summarize some key elements of parton constructions. In Sec. III, we review some well-known properties of spin$\frac{1}{2}$ chains and their description using bosonization. We then describe how a two-dimensional easy-plane antiferromagnet (AFM), valence bond solid (VBS), and Ising-AFM are realized upon introducing interwire couplings and discuss their topological defects. In Secs. IV and Sec. V, we introduce bosonic and fermionic partons, respectively, as combinations of the aforementioned defects. We derive the gauge theory that results when the spin model is rewritten in terms of these nonlocal degrees of freedom and tabulate their symmetries. We then analyze several phases in both their spin and parton representations, with particular attention to the fate of the emergent gauge field in the latter. We conclude with a summary of our results and an outlook on possible extensions in Sec. VI. Finally, the Appendices discuss nonuniversal terms that are required for microscopically exact mappings but do not affect long-distance properties. They also reproduce several known properties of the pertinent gauge theories within concrete wire-based calculations.

\section{PARTON CONSTRUCTION}

In this section, we briefly review partons in the context of interacting spin systems. Two widely used parton constructions are based on the Schwinger-boson or Abrikosov-fermion representations of $\operatorname{spin} \frac{1}{2}$, i.e.,

$$
S^{+}=\psi_{\uparrow}^{\dagger} \psi_{\downarrow}, \quad S^{z}=\frac{1}{2}\left(\psi_{\uparrow}^{\dagger} \psi_{\uparrow}-\psi_{\downarrow}^{\dagger} \psi_{\downarrow}\right) .
$$

Here $\psi_{\sigma}$ are either bosonic or fermionic annihilation operators subject to the constraint of a single parton per site, $\hat{n} \equiv$ $\sum_{\sigma} \psi_{\sigma}^{\dagger} \psi_{\sigma}=1$. The expression of spins through partons has built-in redundancy, i.e., physical spin operators are invariant under phase rotations $\psi_{\sigma} \rightarrow \psi_{\sigma} e^{i \phi}$.

\section{A. Mean-field theory}

The representation (3) allows expressing generic Hamiltonians with $S^{z}$-conserving two-spin interactions as

$$
\begin{aligned}
H= & \sum_{\boldsymbol{r}, \boldsymbol{r}^{\prime}} J_{\boldsymbol{r}, \boldsymbol{r}^{\prime}}^{\perp} \psi_{\uparrow, \boldsymbol{r}}^{\dagger} \psi_{\uparrow, \boldsymbol{r}^{\prime}} \psi_{\downarrow, \boldsymbol{r}^{\prime}}^{\dagger} \psi_{\downarrow, \boldsymbol{r}}+\text { H.c. } \\
& +\sum_{\boldsymbol{r}, \boldsymbol{r}^{\prime}} J_{\boldsymbol{r}, \boldsymbol{r}^{\prime}}^{z} \sum_{\sigma} \psi_{\sigma, \boldsymbol{r}}^{\dagger} \psi_{\sigma, \boldsymbol{r}^{\prime}} \psi_{\sigma, \boldsymbol{r}^{\prime}}^{\dagger} \psi_{\sigma, \boldsymbol{r}} .
\end{aligned}
$$

The absence of quadratic terms implies that $\hat{n}_{\boldsymbol{r}}$ on each site is conserved, i.e., individual partons are immobile. Still, parton hopping may emerge at low energies, which can be captured by mean-field Ansätze such as $\left\langle\psi_{\sigma, r}^{\dagger} \psi_{\sigma^{\prime}, \boldsymbol{r}^{\prime}}\right\rangle=\chi_{\boldsymbol{r}, \boldsymbol{r}^{\prime}} \delta_{\sigma, \sigma^{\prime}}$. The corresponding mean-field Hamiltonian is

$$
H_{\mathrm{MF}}=\sum_{\boldsymbol{r}, \boldsymbol{r}^{\prime}, \sigma}\left(\chi_{\boldsymbol{r}, \boldsymbol{r}^{\prime}} \psi_{\sigma, \boldsymbol{r}^{\prime}}^{\dagger} \psi_{\sigma, \boldsymbol{r}}+\text { H.c. }\right)+\mu \sum_{\boldsymbol{r}} \hat{n}_{\boldsymbol{r}},
$$

where $J_{\boldsymbol{r}, \boldsymbol{r}^{\prime}}^{\perp}$ and $J_{\boldsymbol{r}, \boldsymbol{r}^{\prime}}^{z}$ were absorbed into the definition of $\chi_{\boldsymbol{r}, \boldsymbol{r}^{\prime}}$. We also included a chemical potential that enforces, on average, the now-violated single-occupancy constraint. The mean-field Hamiltonian also breaks the local redundancy: un$\operatorname{der} \psi_{\sigma, r} \rightarrow \psi_{\sigma, r} e^{i \phi_{r}}$ the mean-field parameter acquires a phase

$$
\chi_{\boldsymbol{r}, \boldsymbol{r}^{\prime}} \rightarrow \chi_{\boldsymbol{r}, \boldsymbol{r}^{\prime}} \exp \left[-i\left(\phi_{\boldsymbol{r}}-\phi_{\boldsymbol{r}^{\prime}}\right)\right] .
$$

Both flaws can be remedied by allowing for local fluctuations in $\chi_{r, r^{\prime}}$ and $\mu$ on top of the mean-field values.

\section{B. Compact gauge fluctuations}

The replacement $\chi_{\boldsymbol{r}, \boldsymbol{r}^{\prime}} \rightarrow \chi_{\boldsymbol{r}, \boldsymbol{r}^{\prime}} \exp \left[-i a_{\boldsymbol{r}, \boldsymbol{r}^{\prime}}\right]$ can restore the redundancy if $a_{r, r^{\prime}}$ acquires a shift under phase rotations. Specifically, it must transform like the spatial components of a gauge field, i.e., $a_{\boldsymbol{r}, \boldsymbol{r}^{\prime}} \rightarrow a_{\boldsymbol{r}, \boldsymbol{r}^{\prime}}+\phi_{\boldsymbol{r}}-\phi_{\boldsymbol{r}^{\prime}}$. Allowing fluctuations of $a_{r, r^{\prime}}$ and encoding fluctuations of the chemical potential via a temporal component $\mu \rightarrow \mu+i a_{0, r}$ results in the (Euclidean) action

$$
\begin{aligned}
\mathcal{S}_{\text {parton }}= & \int_{\tau} \sum_{\boldsymbol{r}, \sigma} \psi_{\sigma, \boldsymbol{r}}^{\dagger}\left(\partial_{\tau}-i a_{0, \boldsymbol{r}}-\mu\right) \psi_{\sigma, \boldsymbol{r}} \\
& +\int_{\tau} \sum_{\boldsymbol{r}, \boldsymbol{r}^{\prime}, \sigma}\left(\chi_{\boldsymbol{r}, \boldsymbol{r}^{\prime}} \psi_{\sigma, \boldsymbol{r}^{\prime}}^{\dagger} e^{-i a_{\boldsymbol{r}, \boldsymbol{r}^{\prime}}} \psi_{\sigma, \boldsymbol{r}}+\text { H.c. }\right) .
\end{aligned}
$$

A crucial aspect of this theory is its periodicity in $a_{\boldsymbol{r}, \boldsymbol{r}^{\prime}}$, which permits "monopole" events where the flux $\boldsymbol{\Delta} \times \boldsymbol{a}$ changes by $2 \pi$. The gauge field is thus compact [101]. Equivalently, any induced Maxwell term generated by integrating out $\psi$ inherits the periodicity of the minimal coupling and is of the form $\cos (\boldsymbol{\Delta} \times \boldsymbol{a})$. By contrast, a bare Maxwell term $\propto(\boldsymbol{\Delta} \times \boldsymbol{a})^{2}$, which arises, e.g., in the particle-vortex duality [98-100], would exclude isolated monopoles; for a recent review including modern developments, see Ref. [102]. The same conclusion may be reached after taking the continuum limit by analyzing the microscopic operator that corresponds to the emergent gauge flux. In the present case, it is given by the scalar spin chirality [103]

$$
\vec{S}_{\boldsymbol{r}_{1}} \cdot\left(\vec{S}_{\boldsymbol{r}_{2}} \times \vec{S}_{\boldsymbol{r}_{3}}\right) \underset{\text { cont. limit }}{\longrightarrow} \nabla \times \boldsymbol{a}
$$

which is not conserved in most microscopic spin models. Consequently, monopole events exist in the corresponding gauge theory. By contrast, in particle-vortex duality, the gauge flux is identified with the microscopically conserved boson density. Monopoles are thus absent in that case, i.e., the gauge field is noncompact.

To study compact gauge theories, it is often convenient to separate the gauge field into a monopole-free part $\vec{a}^{0}$ and a singular part $\vec{a}^{\mathcal{M}}$ that contains monopoles of strength $2 \pi q_{i}$ at space-time points $\vec{r}_{i}=\left(\tau_{i}, \boldsymbol{r}_{i}\right)$, i.e.,

$$
\vec{\nabla} \cdot\left(\vec{\nabla} \times \vec{a}^{\mathcal{M}}\right)=2 \pi \sum_{i} q_{i} \delta\left(\vec{r}-\vec{r}_{i}\right) .
$$

This separation is useful, e.g., for assessing the relevance of monopoles in the presence of matter fields $\psi$. The monopolemonopole correlation function is

$$
C_{\vec{r}_{1}-\vec{r}_{2}}^{\mathcal{M}}=\frac{\int \mathcal{D}\left[\psi, a_{\mu}^{0}\right] e^{-S\left[\psi, a_{\mu}^{0}+a_{\mu}^{\mathcal{M}}\right]}}{\int \mathcal{D}\left[\psi, a_{\mu}^{0}\right] e^{-S\left[\psi, a_{\mu}^{0}\right]}}=\left\langle e^{S\left[\psi, a_{\mu}^{0}\right]-S\left[\psi, a_{\mu}^{0}+a_{\mu}^{\mathcal{M}}\right]}\right\rangle_{0} .
$$


TABLE I. Symmetry action on bosonized degrees of freedom, with asterisks denoting antiunitary symmetries $(i \rightarrow-i)$.

\begin{tabular}{llrc}
\hline \hline Symmetry & & $\Phi$ & $\Theta$ \\
\hline$U(\alpha)$ & U(1) spin rot. & $\Phi+\alpha$ & $\Theta$ \\
$\Pi_{y}$ & $\pi$ rot. around $S_{y}$ & $-\Phi+\pi$ & $-\Theta$ \\
$T_{x}: x \rightarrow x+1$ & $x$ translation & $\Phi+\pi$ & $\Theta+\frac{\pi}{2}$ \\
$T_{y}: y \rightarrow y+1$ & $y$ translation & $\Phi+\pi$ & $\Theta+\frac{\pi}{2}$ \\
$\mathcal{I}_{x}: x \rightarrow-x$ & $x$ inversion (site) & $\Phi$ & $-\Theta+\frac{\pi}{2}$ \\
$\mathcal{I}_{y}: y \rightarrow-y+1$ & $\tilde{y}$ inversion & $\Phi+\pi$ & $\Theta+\frac{\pi}{2}$ \\
$\mathcal{T}^{*}: \tau \rightarrow-\tau$ & Time reversal (TR) & $\Phi-\pi$ & $-\Theta$ \\
$\mathcal{T}^{*} \equiv T_{y} \cdot \mathcal{T}$ & AFM-TR & $\Phi$ & $-\Theta-\frac{\pi}{2}$ \\
\hline \hline
\end{tabular}

Here, the singular gauge field $\vec{a}^{\mathcal{M}}$ must satisfy Eq. (9) with two opposite monopoles $q_{1}=-q_{2}=1$, but is otherwise arbitrary.

While conceptually straightforward, the evaluation of $C^{\mathcal{M}}$ based on this formula is a formidable task, only achievable in specific limits. Extensive studies of compact gauge theories in $2+1$ dimensions have found that their infrared behavior falls into one of three categories:

(1) Confining: When all matter fields trivially gapped, the low-energy theory is a pure gauge theory. There, monopoles always proliferate and result in confinement [101].

(2) Gapped: Confinement is avoided when the gauge field becomes massive due to the formation of a condensate (Higgs mass) or a topologically nontrivial insulator (Chern-Simons mass).

(3) Gapless: The presence of gapless matter, e.g., in the form of a large number of Dirac-fermion species or a Fermi surface, can render monopoles irrelevant. Such systems behave like noncompact gauge theories [104-106].

\section{COUPLED-WIRE APPROACH}

Consider a two-dimensional array of antiferromagnetic spin- $\frac{1}{2}$ chains (i.e., wires) with conserved $S^{z}$. The longwavelength properties of each chain, labeled by an integer $y$ and extending along $\hat{x}$, can be efficiently described using Abelian bosonization [107,108]. This framework is, moreover, convenient for including interwire couplings and studying their effect. We thus introduce a pair of conjugate variables $\Theta_{y}(x), \Phi_{y}(x)$ and describe the spin-chain array by a Euclidean path integral $\mathcal{Z}=\int \mathcal{D} \Phi \mathcal{D} \Theta e^{-\mathcal{S}}$. The action $\mathcal{S}$ contains both intrawire terms and couplings between different chains; both will be specified below. In our convention, smooth and staggered components of the microscopic spins $\vec{S}_{\boldsymbol{r}}=\vec{J}_{\boldsymbol{r}}+(-1)^{x+y} \vec{N}_{\boldsymbol{r}}$ are encoded as

$$
\begin{aligned}
J_{\boldsymbol{r}}^{z} & =\frac{1}{\pi} \partial_{x} \Theta_{y}, & & N_{\boldsymbol{r}}^{z} \sim \sin \left(2 \Theta_{y}\right), \\
J_{\boldsymbol{r}}^{+} & \sim e^{i \Phi_{y}} \sin \left(2 \Theta_{y}\right), & & N_{\boldsymbol{r}}^{+} \sim e^{i \Phi_{y}} .
\end{aligned}
$$

The transformation of $\Theta$ and $\Phi$ under microscopic symmetries can be readily deduced from these expressions; we summarize the ones pertinent to this work in Table I.

\section{A. Decoupled spin- $\frac{1}{2}$ chains}

We describe the long-wavelength properties of each spin chain by $\mathcal{L}_{\text {chain }}=\mathcal{L}_{\mathrm{LL}}+\mathcal{L}_{4 \pi}$, where

$$
\begin{aligned}
\mathcal{L}_{\mathrm{LL}} & =\frac{i}{\pi} \partial_{x} \Theta_{y} \partial_{\tau} \Phi_{y}+\frac{v K}{2 \pi}\left(\partial_{x} \Phi_{y}\right)^{2}+\frac{v}{2 \pi K}\left(\partial_{x} \Theta_{y}\right)^{2}, \\
\mathcal{L}_{4 \pi} & =g_{4 \pi} \cos \left(4 \Theta_{y}\right) .
\end{aligned}
$$

The Luttinger-liquid Lagrangian $\mathcal{L}_{\mathrm{LL}}$ is perturbed by the nonlinear $\mathcal{L}_{4 \pi}$, which introduces $4 \pi$ phase slips into $S^{+}$. Its scaling dimension at the Gaussian fixed point $\Delta_{4 \pi}=4 \mathrm{~K}$ determines the nature of the phase. For $K>\frac{1}{2}$ phase slips are irrelevant, and the ground state is gapless, with power-law correlations in $S^{x, y, z}$ and the VBS order parameter

$$
\epsilon_{\boldsymbol{r}}=(-1)^{x} S_{r}^{+} S_{r+\hat{x}}^{-}+\text {H.c. } \sim(-1)^{y} \cos \left(2 \Theta_{y}\right) .
$$

An array of spin chains in this phase is easily destabilized by various types of interwire couplings and will thus be our starting point for accessing two-dimensional phases.

It is, however, useful to briefly review the opposite case of relevant $\mathcal{L}_{4 \pi}$. We begin by introducing a dimensionless coupling constant, whose bare value at the microscopic length $d_{0}$ is $\tilde{g}_{4 \pi} \equiv 16 \pi K d_{0}^{2} g_{4 \pi} / v$. For $K<\frac{1}{2}$ it grows under renormalization and reaches order unity at a length $d_{*}$. For small $\left|\tilde{g}_{4 \pi}\right|$ and $K$, the scaling dimension of the cosine implies $d_{*} \simeq$ $d_{0}\left|\tilde{g}_{4 \pi}\right|^{1 /(4 K-2)}$. Beyond this scale, each field $\Theta_{y}$ becomes trapped around a minimum of the cosine. To describe the low-energy fluctuations we, therefore, expand the cosine to quadratic order and write

$$
\mathcal{L}_{4 \pi}=\frac{\tilde{g}_{4 \pi} v}{16 \pi K d_{0}^{2}} \cos \left(4 \Theta_{y}\right) \rightarrow \frac{v}{2 \pi K d_{*}^{2}}\left(\Theta_{y}-\Theta_{y, 0}^{\left(n_{y}\right)}\right)^{2} .
$$

Here, $\Theta_{y, 0}^{\left(n_{y}\right)}$ denote the minima of the cosines, labeled by the integers $n_{y}$. To identify the ground state, it is sufficient to replace $\Theta_{y} \rightarrow \Theta_{y, 0}^{\left(n_{y}\right)}$ in all observables. For negative $\tilde{g}_{4 \pi}$, the minima are at $\Theta_{y, 0}^{\left(n_{y}\right)}=\pi n_{y} / 2$, and there is VBS order $\epsilon_{\boldsymbol{r}} \propto$ $(-1)^{n_{y}+y}$. For positive $\tilde{g}_{4 \pi}$ we instead have $\Theta_{y, 0}^{\left(n_{y}\right)}=\pi n_{y} / 2+$ $\pi / 4$ reflecting Ising-Néel order, i.e., $N^{z} \propto(-1)^{n_{y}}$. We denote the two possible ground states, $n_{y}$ even and $n_{y}$ odd, by $\mathrm{VBS}_{1}\left(\mathrm{Ising}_{1}\right)$ and $\mathrm{VBS}_{2}$ (Ising 2 ), respectively. In both cases, they are related by $x$ translations (cf. Table I) and one is selected spontaneously when that symmetry is broken. The universal properties of these gapped phases are insensitive to the value of $d_{*}$, which may be viewed as a new parameter that replaces $\tilde{g}_{4 \pi}$.

Consider now a domain wall where the state of the $y_{0}$ th wire is characterized by $n_{y_{0}}$ for $x<x_{0}$ and by $n_{y_{0}}+1$ for $x>x_{0}$. Near the domain wall, $\Theta_{y}$ changes smoothly by $\pi / 2$ over a distance $\sim d_{*}$ to avoid incurring a large elastic energy cost (see Fig. 3). The precise form of this interpolation is not essential for our purposes; a sample function is $\delta \Theta_{\mathrm{DW}}\left(x_{0}\right)=$ $\tan ^{-1}\left[e^{\left(x-x_{0}\right) / d_{*}}\right]$. The total spin associated with introducing an $N$-fold domain wall, $\Theta_{y_{0}}(x) \rightarrow \Theta_{y_{0}}(x)+N \delta \Theta_{\mathrm{DW}}\left(x_{0}\right)$, is

$$
S_{\text {tot }}^{z} \equiv \frac{1}{\pi} \int_{x} \sum_{y} \partial_{x} \Theta_{y}=\frac{N}{\pi} \int_{x} \partial_{x} \delta \Theta_{\mathrm{DW}}=\frac{N}{2} .
$$

Crucially, this value is universal and only depends on the asymptotic behavior of $\Theta$. Moreover, the associated energy 
(a) Low-energy field at a smooth domain wall

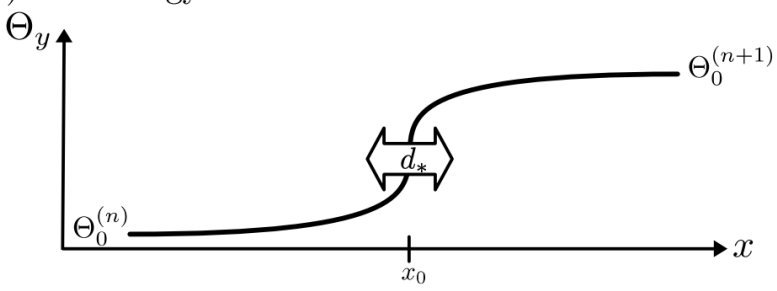

(b) Spins at an atomically sharp VBS domain wall

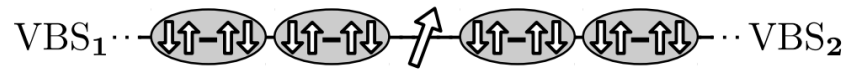

(c) Spins at an atomically sharp Ising domain wall

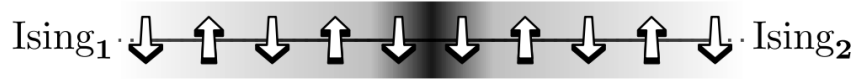

FIG. 3. (a) The fundamental quasiparticle excitations of a onedimensional VBS and an Ising AFM are domain walls between the two degenerate ground states. In both cases, the bosonic variable $\Theta(x)$ changes by $\pi / 2$, from one minimum to the next, over a distance set by the correlation length $d_{*}$. Consequently, such defects carry spin $\frac{1}{2}$ and cost a finite energy $\propto 1 / d_{*}$. When the correlation length is comparable to the lattice spacing, the domain wall can be depicted in terms of microscopic spins as shown in (b) for a VBS, and (c) for an Ising AFM. The spin $\frac{1}{2}$ associated with this excitation becomes readily apparent in this limit.

cost takes a finite nonuniversal value proportional to $d_{*}^{-1}$ (see Appendix A 1 for details). By contrast, a "half domain wall," i.e., $N=\frac{1}{2}$, which formally carries spin $\frac{1}{4}$, costs a finite energy density for all $x>x_{0}$. Consequently, the total energy diverges linearly with the system size, i.e., such configurations are confined.

\section{B. Coupled spin-chain arrays}

To describe two-dimensional phases, we initially neglect $4 \pi$ phase slips in the action of the decoupled array

$$
\mathcal{S}_{\text {decoupled }}=\int_{x, \tau} \sum_{y} \mathcal{L}_{\text {chain }} .
$$

Instead, we perturb $\mathcal{S}_{\text {decoupled }}$ by interwire couplings that drive the system to a new fixed point and analyze the effect of $4 \pi$ phase slips there. The leading coupling terms between neighboring wires that are compatible with the symmetries in Table I are

$$
\begin{aligned}
\mathcal{L}_{t} & =g_{t} \cos \left(\Phi_{y+1}-\Phi_{y}\right), \\
\mathcal{L}_{u} & =g_{u} \cos \left(2 \Theta_{y+1}+2 \Theta_{y}\right) .
\end{aligned}
$$

A third cosine, $\cos \left(2 \Theta_{y+1}-2 \Theta_{y}\right)$, has the same scaling dimension at the decoupled fixed point as the one in $\mathcal{L}_{u}$. However, it can be obtained by combining the latter with $4 \pi$ phase slips and thus need not be treated independently. The cosines in Eq. (18) compete to drive the wire array into different symmetry-broken states (see Fig. 4). Their topological defects are crucial for relating spins to the bosonic or fermionic partons. We, therefore, briefly discuss how their key properties arise within the coupled-wire framework.

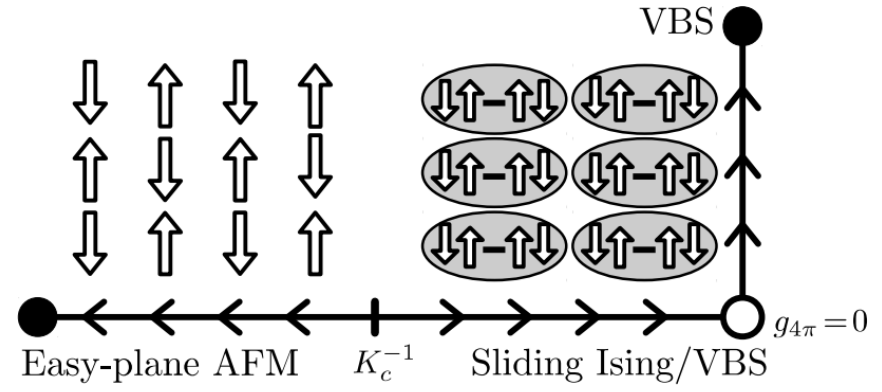

FIG. 4. The lowest-order coupling terms between neighboring spin chains drive the two-dimensional array either into an easy-plane AFM or into a gapless "sliding Ising/VBS" state. Phase slips are irrelevant in the former, but strongly relevant in the latter, where they lead to a fully gapped VBS phase.

\section{Easy-plane antiferromagnet (AFM)}

Consider $K \gg 1$ such that $\mathcal{L}_{t}$ of Eq. (18a) is strongly relevant while $\mathcal{L}_{u}$ of Eq. (18b) flows to zero. As in Sec. III A, we introduce a dimensionless coupling constant with bare value $\tilde{g}_{t} \equiv \pi d_{0}^{2} g_{t} / v K<0$. The flow of $\tilde{g}_{t}$ to strong coupling permits us to replace

$$
\mathcal{L}_{t}=\frac{v K \tilde{g}_{t}}{\pi d_{0}^{2}} \cos \left(\Phi_{y+1}-\Phi_{y}\right) \rightarrow \frac{v K}{2 \pi d_{*}^{2}}[\Delta \Phi]_{\tilde{y}}^{2},
$$

where $\Delta_{\tilde{y}, y^{\prime}} \equiv\left(\delta_{y+1, y^{\prime}}-\delta_{y, y^{\prime}}\right)$ is the discrete $y$ derivative, naturally centered on a dual wire $\tilde{y}=y+\frac{1}{2}$. The scaling dimension of the cosine at the decoupled fixed point implies $d_{*} \simeq d_{0}\left|\tilde{g}_{t}\right|^{1 /\left(K^{-1}-4\right)}$ for small $\tilde{g}_{t}$, but that is not essential for our purposes.

On a finite array of $N_{\mathrm{w}}$ wires, only $N_{\mathrm{w}}-1$ of the differences $\Delta \Phi$ are linearly independent, and the system remains gapless. The missing linear combination $N_{\mathrm{w}}^{-1} \sum_{y} \Phi_{y}$ cannot be pinned due to the global U(1) spin-rotation symmetry (cf. Table I). This property reflects the presence of a Goldstone mode due to spontaneous U(1) symmetry breaking. The effective action

$$
\mathcal{L}_{\mathrm{SF}}=\mathcal{L}_{\mathrm{LL}}+\frac{v K}{2 \pi d_{*}^{2}}[\Delta \Phi]_{\tilde{y}}^{2}
$$

can be brought to a more familiar form by performing the Gaussian integral over $\Theta_{y}$. Additionally taking the continuum limit $d_{*}^{-1} \Delta \rightarrow \partial_{y}$ results in

$$
\mathcal{S}_{\mathrm{SF}}=\frac{K}{2 \pi v} \int_{\tau, x, y}\left[\left(\partial_{\tau} \Phi\right)^{2}+v^{2}(\nabla \Phi)^{2}\right] .
$$

It is straightforward to verify that $4 \pi$ phase slips are irrelevant at this new fixed point and that it exhibits Néel order $\left\langle N_{r}^{+}\right\rangle \neq 0$.

For the topological defects, the periodicity of $\mathcal{L}_{t}$ is paramount. The number of domain walls in a given cosine is $N_{\tilde{y}} \equiv \frac{1}{2 \pi} \int_{x}\left[\Delta \partial_{x} \Phi\right]_{\tilde{y}} \sim \frac{1}{2 \pi} \oint_{\Gamma} d \boldsymbol{l} \cdot \nabla \Phi$, where $\Gamma$ encloses the plaquette containing $\tilde{y}$. Consequently, $N_{\tilde{y}}$ is precisely the number of magnetic vortices contained within this plaquette or, equivalently, on the dual wire. A sample configuration that contains an isolated vortex is $\delta \Phi_{y}^{\text {vortex, } \xi}(x)=$ $\arg \left[\left(x-x_{0}\right) / \xi+i\left(y-\tilde{y}_{0}\right)\right]$. According to Eq. (20), its energy exhibits the familiar logarithmic divergence with system size 
Isotropic vortex $(\xi=1) \quad$ Deformed vortex $\left(\xi=\frac{1}{8}\right)$

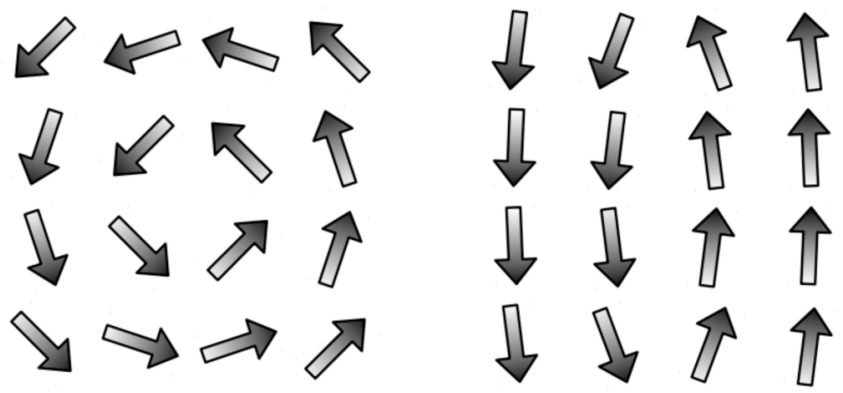

FIG. 5. Two vortex configurations, shown in terms of the Néel vector $\vec{N}$, may have significant differences in trial energies but are topologically equivalent. For formal manipulations the precise choice is unimportant, and the more anisotropic limit $\xi \rightarrow 0$ turns out to be the most convenient in the present case.

(see Appendix A 2). For the formal manipulations below, it is convenient to use the $\xi \rightarrow 0$ limit of the above expression, i.e.,

$$
\delta \Phi_{y}^{\operatorname{vortex}}(x)=\pi \operatorname{sgn}\left(\tilde{y}_{0}-y\right) H\left(x-x_{0}\right),
$$

with $H(x)$ the Heaviside step function. In this configuration, all phase winding is concentrated along one-dimensional lines rather than uniformly spread as in the isotropic one. A vortex in the form of Eq. (22) is created by the operator

$$
V_{\tilde{y}_{0}}^{\dagger}\left(x_{0}\right) \equiv \exp \left[i \sum_{y} \operatorname{sgn}\left(y-\tilde{y}_{0}\right) \Theta_{y}\left(x_{0}\right)\right]
$$

which satisfies $V^{\dagger} \Phi V=\Phi+\delta \Phi^{\text {vortex }}$. The same form of this vortex operator was used in Ref. [96] to implement dualities between bosons and Dirac fermions on coupled-wire arrays. Vortex configurations in both limits are illustrated in Fig. 5.

\section{Intrawire valence bond solid (VBS)}

For $K \ll \frac{1}{4}$, it is $\mathcal{L}_{u}$ of Eq. (18b) that is strongly relevant while $\mathcal{L}_{t}$ of Eq. (18a) flows to zero. We focus on positive $g_{u}$ and express it in terms of a dimensionless coupling constant. To describe low energies, we replace

$\mathcal{L}_{u}=\frac{v \tilde{g}_{u}}{4 \pi K d_{0}^{2}} \cos \left(2 \Theta_{\tilde{y}+1}+2 \Theta_{\tilde{y}}\right) \rightarrow \frac{v}{2 \pi K d_{*}^{2}}\left([S \Theta]_{\tilde{y}}-\frac{\pi}{2}\right)^{2}$,

where $S_{\tilde{y}, y^{\prime}} \equiv\left(\delta_{y+1, y^{\prime}}+\delta_{y, y^{\prime}}\right)$ is naturally centered on a dual wire. This Lagrangian is identical to Eq. (19) upon replacing $\Theta_{y} \leftrightarrow(-1)^{y}\left(\Phi_{y}+\pi y / 2\right)$ and $K \leftrightarrow K^{-1}$, which does not affect the kinetic interwire terms. Consequently, the analysis of the easy-plane AFM carries over.

The ground state exhibits Ising-Néel and/or VBS orders according to

$$
\left\langle N^{z}\right\rangle \propto \sin \left(2 \Theta_{0}\right), \quad\langle\epsilon\rangle \propto \cos \left(2 \Theta_{0}\right),
$$

with a spontaneously chosen $\Theta_{0}$. Around a topological defect, $\Theta_{0}$ winds smoothly by $\pi$. Such defects are illustrated in Fig. 6 and will be referred to as dislocations. Around them, the state of the system changes from Ising first to $\mathrm{VBS}_{1}$, then to Ising ${ }_{2}$, next to $\mathrm{VBS}_{2}$, and finally returns to the Ising configuration. $^{1}$ The creation operator in the extreme vertically deformed

\footnotetext{
${ }^{1}$ These defects share several features with $\mathbb{Z}_{4}$ vortices in columnar VBS states [119]. There, the two Ising phases are replaced by interwire valence bond states $\mathrm{VBS}_{3,4}$ that arise as $\pi / 2$ rotations of the $\mathrm{VBS}_{1,2}$. The $\mathrm{VBS}_{3,4}$ configurations interchange under $T_{y}$ and are invariant under $\mathcal{T}^{\prime}$, just like Ising ${ }_{1,2}$. However, their transformations under $T_{x}$ and $\mathcal{T}$ are different. Interwire VBS states in wire models are described in Sec. IV B 3. Unfortunately, operators that would create $\mathbb{Z}_{4}$ VBS vortices are not readily accessible in this framework.
}

\section{Horizontal deformation}

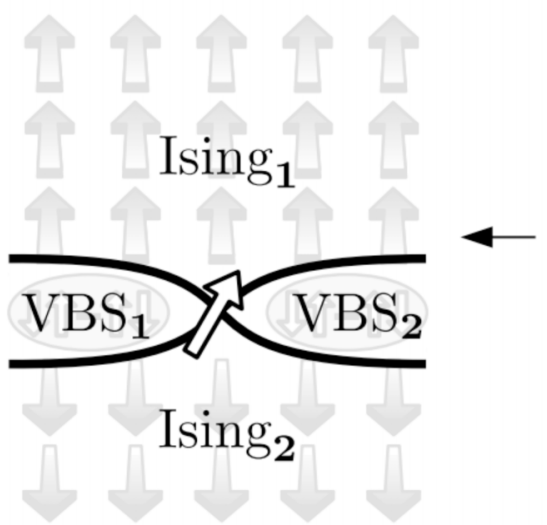

Isotropic dislocation

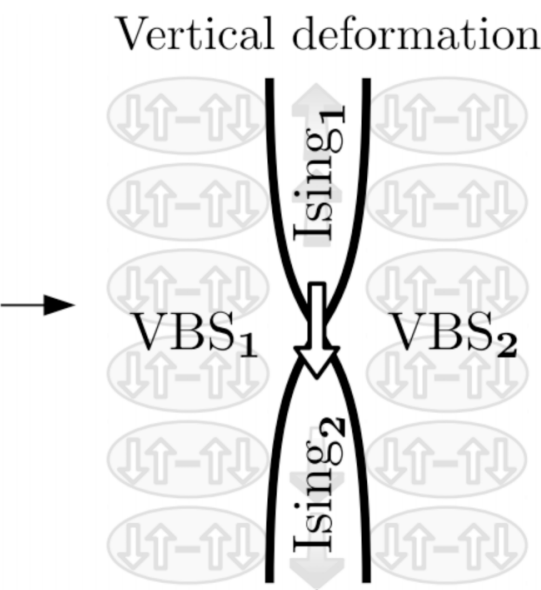

FIG. 6. Four fundamental domain boundaries between $\mathrm{VBS}_{1}, \mathrm{Ising}_{1}, \mathrm{VBS}_{2}$, and $\operatorname{Ising}_{2}$ regions terminate in a dislocation that carries spin $\frac{1}{2}$. This topological property holds irrespective of the detailed configuration. It becomes apparent in the strongly anisotropic limits, where either the Ising or the VBS phases extend only along a one-dimensional line. There, the properties of the dislocation can be inferred from the one-dimensional domain walls illustrated in Fig. 3. In our formulation, bosonic partons in a specific gauge are introduced as the extreme vertically deformed dislocations; they carry spin $\frac{1}{2}$ and live on dual wires. 
limit is

$$
b_{\tilde{y}_{0}}^{\dagger}\left(x_{0}\right) \equiv \exp \left[\frac{i}{2} \sum_{y} \operatorname{sgn}\left(y-\tilde{y}_{0}\right)(-1)^{y} \Phi_{y}\left(x_{0}\right)\right],
$$

which satisfies $b^{\dagger} \Theta b=\Theta-(-1)^{y} \delta \Theta^{\text {vortex }}$ with $\delta \Theta^{\text {vortex }}$ as in Eq. (22).

At this fixed point (referred to as sliding Ising/VBS in Fig. 4), the previously neglected $4 \pi$ phase-slip term, Eq. (13), is strongly relevant. Its flow to strong coupling eliminates the zero-energy mode $\Theta_{y}(x) \rightarrow \Theta_{y}(x)+(-1)^{y} \alpha$ and results in a gapped phase. As in the case of decoupled chains, a VBS is realized for negative $g_{4 \pi}$, while positive $g_{4 \pi}$ leads to an IsingAFM. In both phases, the energy cost of creating an isolated dislocation diverges linearly with the system size (see Appendix A 3). Tightly bound dislocation-antidislocation pairs are thus the fundamental low-energy excitations. When the two are located on neighboring plaquettes, they form precisely the staggered component of the microscopic spin operators

$$
b_{y-1 / 2}^{\dagger} b_{y+1 / 2}= \begin{cases}N_{y}^{+}, & y \text { even } \\ N_{y}^{-}, & y \text { odd } .\end{cases}
$$

Notice the similarity to the parton decomposition $S^{+}=\psi_{\uparrow}^{\dagger} \psi_{\downarrow}$ in Eq. (3). ${ }^{2}$ To make this connection manifest, we introduce the redundant label $\sigma=\uparrow, \downarrow$ for odd and even $\tilde{y}$, respectively. We then define $b_{\tilde{y}, \sigma}^{\dagger}=e^{i \varphi_{\tilde{y}, \sigma}}$ and the associated dislocation density $\rho_{\tilde{y}, \sigma}=\frac{1}{\pi} \partial_{x} \theta_{\tilde{y}, \sigma}$ with

$$
\begin{aligned}
\varphi_{\tilde{y}, \sigma} & =\frac{1}{2} \sum_{y} \operatorname{sgn}(y-\tilde{y})(-1)^{y} \Phi_{y}, \\
\theta_{\tilde{y}, \sigma} & =\sigma\left(\Theta_{y+1}+\Theta_{y}\right) .
\end{aligned}
$$

The total number of $b_{\sigma}$ bosons (dislocations centered on even or odd dual wires) is

$$
Q_{\sigma} \equiv \int_{x} \sum_{\tilde{y}} \rho_{\tilde{y}, \sigma}=\frac{1}{\pi} \int_{x} \sum_{\tilde{y}} \partial_{x} \theta_{\tilde{y}, \sigma}=\sigma S_{\text {total }}^{z},
$$

with $S_{\text {total }}^{z}$ as defined in Eq. (16). Crucially, when $S_{\text {total }}^{z}$ is microscopically conserved, then the number of each of these boson species is separately conserved.

To conclude the discussion of this phase, we want to point out a close connection between dislocations and magnetic vortices. Consider a vortex in $b_{\sigma}$ only, but not in $b_{\bar{\sigma}}$. To

\footnotetext{
${ }^{2}$ There are also notable differences: (i) The dislocation operators $b_{\uparrow(\downarrow)}$ reside on half the wires while Eq. (3) yields operators $\psi_{\sigma}$ on all wires. Nevertheless, since $\psi_{\sigma}$ are constrained while $b_{\sigma}$ are $u n$ constrained, the number of degrees of freedom agrees. (ii) Interwire hopping of $b_{\sigma}$ is represented by a local operator [cf. Eq. (31a)] while for $\psi_{\sigma}$ such hopping is absent on the lattice scale. There, parton kinetic terms may emerge at long wavelengths, described by fluctuations around a nontrivial saddle point. Dislocations should thus be viewed as representing the low-energy progeny of the lattice operators $\psi_{\sigma}$. (iii) The operators $b_{\sigma}$ are formally assigned to reside between wires while $\psi_{\sigma}$ live on the original lattice sites (different decompositions are of course possible, see, e.g., Ref. [120]). However, since hopping of individual $\psi_{\sigma}$ at the lattice scale is a mean-field artifact [see also (i) and (ii)], this is not a significant difference.
}

construct its creation operator, one need only replace $\Theta \rightarrow \theta_{\sigma}$ in Eq. (23). Explicitly, we introduce

$$
\tilde{\varphi}_{\sigma, \tilde{y}} \equiv \begin{cases}-\sum_{\tilde{y}^{\prime} \text { even }} \operatorname{sgn}\left(\tilde{y}-\tilde{y}^{\prime}\right) \theta_{\sigma, \tilde{y}^{\prime}}, & \sigma=\downarrow(\tilde{y} \text { odd }) \\ -\sum_{\tilde{y}^{\prime} \text { odd }} \operatorname{sgn}\left(\tilde{y}-\tilde{y}^{\prime}\right) \theta_{\sigma, \tilde{y}^{\prime}}, & \sigma=\uparrow(\tilde{y} \text { even }) .\end{cases}
$$

Using Eqs. (23) and (28b) we find that $V_{2 \tilde{y}}=e^{i \tilde{\varphi}_{\uparrow, 2 \tilde{y}}}$ and $V_{2 \tilde{y}+1}=e^{i \tilde{\varphi}_{\downarrow, 2 \tilde{y}+1}}$. Dislocations are thus dual to magnetic vortices in this precise sense.

\section{BOSONIC PARTONS FROM COUPLED WIRES}

To access a wider range of phases, including QSLs, we now develop a dual description of the coupled-wire model in terms of the topological defects described above. Such a reformulation of spin- $\frac{1}{2}$ models in terms of magnetic vortices has already been used in Ref. [109] to access, e.g., a putative "deconfined" quantum critical point between Néel and VBS orders. To connect coupled-wire and parton techniques, we instead focus on dislocations and argue that they form a bosonic-parton representation of the microscopic spins.

As discussed at the end of the previous section, $b_{\sigma}$ possesses several of the relevant bosonic-parton properties. Moreover, while the combination $b_{\uparrow}^{\dagger} b_{\downarrow}$ is local in terms of microscopic operators, and carries spin 1 , individual $b_{\sigma}$ are nonlocal and do not have well-defined spin. This property closely relates to the local gauge redundancy of conventional parton decompositions discussed in Sec. II B. Even though $b_{\sigma}$ are nonlocal, the interwire couplings introduced in Eq. (18) remain local under the mapping. Translating them via Eq. (28), we find an intraspecies nearest-neighbor tunneling term and an umklapp term

$$
\begin{aligned}
& \mathcal{L}_{t}=g_{t} \cos \left(\varphi_{\tilde{y}+2, \sigma}-\varphi_{\tilde{y}, \sigma}\right) \sim b_{\tilde{y}+2, \sigma}^{\dagger} b_{\tilde{y}, \sigma}+\text { H.c. }, \\
& \mathcal{L}_{u}=g_{u} \cos \left(2 \theta_{\tilde{y}, \sigma}\right) \sim b_{\tilde{y}, \sigma}^{\dagger} b_{\tilde{y}, \sigma} U(x),
\end{aligned}
$$

where $U(x)=U(x+1)$ is a weak periodic potential (see, e.g., Ref. [107]). The periodicity of $\mathcal{L}_{u}$ implies that $b_{\sigma}$ are both at unit filling. Consequently, the densities of $b_{\uparrow}$ and $b_{\downarrow}$ are the same as in conventional parton constructions (cf. Sec. II). By contrast, the intrawire interactions of the microscopic spin chains are nonlocal in bosonic-parton variables. They have a natural interpretation in terms of an emergent gauge field, as we will now explain.

\section{A. Gauge theory}

To derive the action governing the bosonic partons, we invert the mapping in Eq. (28) and insert them into the microscopic wire-array model in Eq. (17). It is convenient to include, on top of $\mathcal{L}_{\text {chain }}$, the symmetry-allowed quadratic interwire couplings

$$
\mathcal{L}_{\text {inter }}=\frac{u_{B}}{2 \pi}\left[S \partial_{x} \Theta\right]_{\tilde{y}}^{2}+\frac{u_{V}}{8 \pi}\left[\Delta \partial_{x} \Phi\right]_{\tilde{y}}^{2} .
$$

In the limit of weakly coupled wires, these arise as the leading renormalizations of the kinetic energy due to Eq. (18), but in generic cases, $u_{B}$ and $u_{V}$ should be viewed as independent parameters.

While $\mathcal{L}_{t}, \mathcal{L}_{u}$, and $\mathcal{L}_{\text {inter }}$ [Eqs. (18) and (32)] are local in terms of partons, the intrawire interactions $\mathcal{L}_{\text {chain }}$ are highly 
nonlocal. This nonlocality can be encoded exactly through an emergent gauge field $\vec{a}$, just as in the case of the boson-vortex duality (see Appendix B for details) [96]. We express the resulting gauge theory as $\mathcal{S}_{b}=\int_{x, \tau} \sum_{\tilde{y}}\left[\mathcal{L}_{b}+\mathcal{L}_{\text {Maxwell }}+\mathcal{L}_{\text {int }}\right]$. The first two contributions contain parton and gauge-field kinetic terms as well as the coupling between the two, i.e.,

$$
\begin{aligned}
\mathcal{L}_{b}= & \frac{i}{\pi} \partial_{x} \theta_{\tilde{y}, \sigma}\left(\partial_{\tau} \varphi_{\tilde{y}, \sigma}-a_{0, \tilde{y}}\right)+\frac{v_{B}}{2 \pi}\left(\partial_{x} \varphi_{\tilde{y}, \sigma}-a_{1, \tilde{y}}\right)^{2} \\
& +\frac{u_{B}}{2 \pi}\left(\partial_{x} \theta_{\tilde{y}, \sigma}\right)^{2}, \\
\mathcal{L}_{\text {Maxwell }}= & \frac{\kappa \tilde{v}}{4 \pi}\left[\Delta a_{1}\right]_{y}^{2}+\frac{\kappa}{4 \pi \tilde{v}}\left[\Delta a_{0}\right]_{y}^{2} .
\end{aligned}
$$

The parameters $v_{B}, u_{B}, \tilde{v}$, and $\kappa$ are nonuniversal. For their expression in terms of microscopic spin-chain parameters, see Appendix B. There, we also specify the last term $\mathcal{L}_{\text {int }}$, which contains exponentially decaying interwire density-density and current-current interactions.

The gauge-field Lagrangian $\mathcal{L}_{\text {Maxwell }}$ describes an anisotropic Maxwell term in the $a_{2}=0$ gauge but missing the $\propto\left(\partial_{\tau} a_{1, \tilde{y}}-\partial_{x} a_{0, \tilde{y}}\right)^{2}$ contribution. Such a term will be generated upon integrating out matter fields at high energies. We demonstrate this in Sec. IV B 1 for the case of trivially gapped partons. Finally, it is instructive to express the bosonized model in terms of $b_{\sigma}$ as

$$
\mathcal{L}_{b}^{\prime}=b_{\tilde{y}, \sigma}^{\dagger}\left(\partial_{\tau}-i a_{0}-\mu\right) b_{\tilde{y}, \sigma}+\frac{v_{B}}{2 \rho_{0}}\left|\left(\partial_{x}-i a_{x}\right) b_{\tilde{y}, \sigma}\right|^{2},
$$

where $\rho_{0}=\pi$ is the boson density. This Lagrangian has the expected structure for bosonic partons [cf. Eq. (7)].

\section{Monopoles}

The $4 \pi$ phase slips in Eq. (13) are also nonlocal in bosonicparton variables. To interpret them, we introduce the operator

$$
\begin{aligned}
\widehat{\mathcal{M}}_{\boldsymbol{r}} & \equiv \exp \left[2 i(-1)^{y} \Theta_{y}\right]=\exp \left[i \sum_{\tilde{y}} \operatorname{sgn}(y-\tilde{y}) \theta_{\tilde{y}}\right] \\
& =\exp \left[-i \tilde{\varphi}_{\sigma, \tilde{y}}-i \tilde{\varphi}_{\bar{\sigma}, \tilde{y}-1}\right] .
\end{aligned}
$$

For both bosonic partons, $\widehat{\mathcal{M}}_{\boldsymbol{r}_{0}} b_{\sigma}^{\dagger} \widehat{\mathcal{M}}_{\boldsymbol{r}_{0}}^{\dagger} \rightarrow b_{\sigma}^{\dagger} e^{i \alpha\left(\boldsymbol{r}-\boldsymbol{r}_{0}\right)}$, where $\alpha(\boldsymbol{r})$ winds counterclockwise by $2 \pi$ around the origin. $\widehat{\mathcal{M}}_{\boldsymbol{r}}$ can thus be viewed as the insertion of a $2 \pi$ monopole in the emergent gauge field at position $\boldsymbol{r}$. Since it is odd under lattice translations (cf. Table I), $4 \pi$ monopoles created by $\widehat{\mathcal{M}}_{r}^{2}$ are the minimal ones allowed by symmetries.

It is useful to disentangle the monopoles from the matter fields. We, therefore, write $\mathcal{M}^{\dagger}=e^{i \phi_{\mathcal{M}}}$ and replace Eq. (13) by

$$
\mathcal{L}_{4 \pi}=\frac{g_{4 \pi}}{2}\left[\mathcal{M}_{\boldsymbol{r}}^{2}+\mathcal{M}_{\boldsymbol{r}}^{2 \dagger}\right]-\frac{i}{\pi} \partial_{x} \lambda_{y}\left(\phi_{\mathcal{M}, y}-\tilde{\varphi}_{\sigma, \tilde{y}}-\tilde{\varphi}_{\bar{\sigma}, \tilde{y}-1}\right),
$$

where $\lambda$ is a Lagrange multiplier, and $\phi_{\mathcal{M}}$ is now an independent variable in the functional integral. A simple shift $a_{0, \tilde{y}} \rightarrow$ $a_{0, \tilde{y}}-\sum_{y^{\prime}} \operatorname{sgn}\left(y^{\prime}-\tilde{y}\right) \lambda_{y}$ decouples the Lagrange multiplier from the matter fields and moves it into the gauge-field action. Integrating it out then yields our final form of the monopole
TABLE II. The action of microscopic symmetries on bosonic partons and their interpretation in the parton gauge theory. Antiunitary symmetries $(i \rightarrow-i)$ are indicated by asterisks.

\begin{tabular}{lrrrl}
\hline \hline $\begin{array}{l}\text { Microscopic } \\
\text { symmetry }\end{array}$ & $b_{\uparrow}^{\dagger} b_{\downarrow}$ & $\rho_{\sigma}$ & $\mathcal{M}$ & $\begin{array}{l}\text { Parton } \\
\text { interpretation }\end{array}$ \\
\hline$U(\alpha)$ & $e^{i \alpha} b_{\uparrow}^{\dagger} b_{\downarrow}$ & $\rho_{\sigma}$ & $\mathcal{M}$ & $\begin{array}{l}\text { Global } Q_{\uparrow}-Q_{\downarrow} \\
\text { gauge transform. }\end{array}$ \\
& $-b_{\downarrow}^{\dagger} b_{\uparrow}$ & $-\rho_{\sigma}$ & $\mathcal{M}^{\dagger}$ & $\begin{array}{l}\text { Particle hole (PH) } \\
\Pi_{y}\end{array}$ \\
$T_{x}: x \rightarrow x+1$ & $-b_{\uparrow}^{\dagger} b_{\downarrow}$ & $\rho_{\sigma}$ & $-\mathcal{M}$ & $x$ translation \\
$T_{y}: \tilde{y} \rightarrow \tilde{y}+1$ & $-b_{\uparrow}^{\dagger} b_{\downarrow}$ & $-\rho_{\bar{\sigma}}$ & $-\mathcal{M}^{\dagger}$ & PH + spin-flip \\
$\mathcal{I}_{x}: x \rightarrow-x$ & $b_{\uparrow}^{\dagger} b_{\downarrow}$ & $-\rho_{\sigma}$ & $-\mathcal{M}^{\dagger}$ & $x$ inversion \\
$\mathcal{I}_{y}: \tilde{y} \rightarrow-\tilde{y}$ & $-b_{\uparrow}^{\dagger} b_{\downarrow}$ & $\rho_{\sigma}$ & $-\mathcal{M}^{\dagger}$ & $\tilde{y}$ inversion \\
$\mathcal{T}^{*}: \tau \rightarrow-\tau$ & $-b_{\downarrow}^{\dagger} b_{\uparrow}$ & $-\rho_{\sigma}$ & $\mathcal{M}^{\dagger}$ & PH* \\
$\mathcal{T}^{* *}: \tau \rightarrow-\tau$ & $b_{\downarrow}^{\dagger} b_{\uparrow}$ & $\rho_{\bar{\sigma}}$ & $-\mathcal{M}^{\dagger}$ & TR \\
\hline \hline
\end{tabular}

\section{Lagrangian}

$$
\mathcal{L}_{\mathcal{M}}=\frac{g_{\mathcal{M}}}{2}\left[\mathcal{M}_{\vec{r}}^{2}+\mathcal{M}_{\vec{r}}^{2 \dagger}\right]-\frac{\kappa}{4 \pi \tilde{v}}\left[\Delta a_{0}-\frac{i \tilde{v}}{\kappa} \partial_{x} \phi_{\mathcal{M}}\right]_{y}^{2},
$$

with parameters $\kappa, \tilde{v}$ as in Eq. (34) and where we have relabeled $g_{4 \pi} \rightarrow g_{\mathcal{M}}$. For $g_{\mathcal{M}}=0$, the Gaussian integral over $\phi_{\mathcal{M}}$ is a complete square and does not affect any gaugefield or matter correlation function. Alternatively, the second term in $\mathcal{L}_{\mathcal{M}}$ may be viewed as a "bare" kinetic energy for the monopoles. Their interaction with the gauge field, which is not of minimal coupling form, introduces additional terms $\sim\left(\partial_{\tau} \phi_{\mathcal{M}}\right)^{2}$ and $\sim\left(\Delta \phi_{\mathcal{M}}\right)^{2}$ upon integrating out shortdistance gauge fluctuations. The low-energy behavior of the monopoles can, of course, be qualitatively different, depending on the phase of the gauge theory [see, e.g., Eq. (52)].

\section{Symmetries}

To complete the description of the parton-gauge theory, we now specify how the microscopic symmetries of Table I are implemented. The straightforward application of the mapping between spins and partons leads to the symmetry properties summarized in Table II. Additionally, we introduce an external probing field $\vec{A}$ that minimally couples to the conserved $S^{z}$ of the microscopic spins. In its presence, the theory for the decoupled spin chain is augmented to $\mathcal{L}_{\text {chain }} \rightarrow \mathcal{L}_{\text {chain }}+\mathcal{L}_{A}$ with

$$
\mathcal{L}_{A}=-\frac{i}{\pi} \partial_{x} \Theta_{y} A_{0, y}-\frac{v K}{\pi} \partial_{x} \Phi_{y} A_{1, y}+\frac{v K}{2 \pi} A_{1, y}^{2} .
$$

Rederiving the bosonic-parton action with these terms (see Appendix B) amounts, at lowest order in $\Delta$, to replacing $a_{\mu, \tilde{y}} \rightarrow a_{\mu, \tilde{y}}-\frac{1}{4}(-1)^{\tilde{y}}\left[S A_{\mu}\right]_{\tilde{y}}$ in Eqs. (33) and (35).

\section{Alternative perspective}

An alternative route to the parton gauge theory begins with rewriting the wire array in terms of magnetic vortices [see Eq. (23)]. On a lattice, these vortices experience an average flux of $\pi$ per plaquette $[110,111]$. Their band structure thus exhibits two valleys, which amounts to two vortex flavors at low energies. To see how this is reflected in the wire framework, consider the interwire couplings of Eq. (18). The XY spin exchange $\mathcal{L}_{t}$ translates into $2 \pi$ phase slips for the vortex 
$V_{\tilde{y}}$ while

$$
\mathcal{L}_{u}=g_{u} V_{\tilde{y}+2}^{\dagger} V_{\tilde{y}}+\text { H.c. }
$$

The wire-array model $\mathcal{L}_{\mathrm{LL}}+\mathcal{L}_{u}+\mathcal{L}_{t}$ can thus be equivalently expressed in terms of two separately conserved vortex flavors that reside on even or odd dual wires. Moreover, all vortices are coupled to the same noncompact gauge field $\vec{a}_{\text {vortex }}$ whose flux represents the conserved $S^{z}$. (The derivation of this gauge theory within the wire framework is identical to the one performed above for bosonic partons.) Performing separate dualities for the two vortex flavors results in two species of bosonic partons (cf. the final paragraph of Sec. III B 2) coupled to a single gauge field $\vec{a}$ that is, likewise, noncompact. Its flux corresponds to the difference between the numbers of $V_{\text {even }}$ and $V_{\text {odd }}$ vortices. This difference ceases to be conserved in the presence of the $4 \pi$ phase slips. Indeed,

$$
\mathcal{L}_{4 \pi}=g_{4 \pi} V_{2 \tilde{y}} V_{2 \tilde{y}} V_{2 \tilde{y}+1}^{\dagger} V_{2 \tilde{y}+1}^{\dagger}+\text { H.c. }
$$

allows vortex pairs to switch their flavor. Such processes change the flux of the gauge field $\vec{a}$ by $4 \pi$ and, thereby, render it compact.

\section{B. Phases of bosonic partons and spins}

To demonstrate the generality of the formalism developed above, we now apply it to several concrete examples. In the spirit of most parton constructions, we primarily consider phases that are trivial at the mean-field level, i.e., in the absence of gauge fluctuations. These include Mott insulators, superfluids, and integer quantum Hall states. Coupled-wire models that realize such phases are either known in the literature or can be constructed relatively easily [51,55,70,93]. The mapping in Eq. (28) then immediately provides a corresponding coupled-wire model in terms of the microscopic spin variables. We carefully examine both representations of each phase, in terms of partons and of spins, and demonstrate their equivalence.

We analyze the parton models as follows: First, we determine the ground state and excitations of $\mathcal{L}_{b}+\delta \mathcal{L}$ at the mean-field level, i.e., by treating $\vec{a}$ as static. Second, we reintroduce the gauge-field dynamics and determine how they are affected by the matter fields. If the gauge field remains massless, we analyze the effect of monopoles. Third, we examine the quasiparticle content of the gauge theory. Finally, we perform a conventional analysis of the equivalent coupled-wire model in terms of the microscopic spin variables $\mathcal{L}=\mathcal{L}_{\mathrm{LL}}+\delta \mathcal{L}[\Phi, \Theta]$, and verify that its properties match the ones obtained from the parton gauge theory.

\section{Mott insulator and intrawire VBS}

Mean field. A trivial Mott insulator of partons forms when the umklapp term of Eq. (31b) gaps the bosons on each wire separately. This interaction does not contain $\varphi_{\tilde{y}, \sigma}$, which can, therefore, be integrated out trivially. The parton Mott insulator is thus described by

$$
\begin{aligned}
\mathcal{L}_{\text {Mott }}= & \frac{1}{2 \pi v_{B}}\left(\partial_{\tau} \theta_{\tilde{y}, \sigma}\right)^{2}+\frac{u_{B}}{2 \pi}\left(\partial_{x} \theta_{\tilde{y}, \sigma}\right)^{2}+g_{u} \cos \left(2 \theta_{\tilde{y}, \sigma}\right) \\
& +\frac{i}{\pi} \theta_{\tilde{y}, \sigma}\left(\partial_{x} a_{0, \tilde{y}}-\partial_{\tau} a_{1, \tilde{y}}\right) .
\end{aligned}
$$

At sufficiently long length scales, each field $\theta_{\tilde{y}, \sigma}$ becomes trapped around the minima of the cosine, as in Sec. III A. Integrating out the massive fluctuations around these minima yields $\mathcal{L}_{\text {ind }} \propto\left(\partial_{\tau} a_{1, \tilde{y}}-\partial_{x} a_{0, \tilde{y}}\right)^{2}$, which modifies the dielectric constant of the (static) gauge field $\vec{a}$. The periodicity of the cosine implies that $\theta_{\tilde{y}, \sigma}$ winds by $\pi$ at a fundamental domain wall. Such a configuration describes an isolated parton, created by the operator $b_{\sigma}^{\dagger}$. At the mean-field level, these spin- $\frac{1}{2}$ excitations are the elementary quasiparticles.

Gauge fluctuations. We now reinstate the dynamics of the gauge field and supplement its bare action, Eq. (34), by $\mathcal{L}_{\text {ind }}$. The effective Lagrangian is thus

$$
\mathcal{L}_{\mathrm{MW}}=\frac{\kappa}{4 \pi \tilde{v}}\left\{\left[\Delta a_{0}\right]_{y}^{2}+\tilde{v}^{2}\left[\Delta a_{1}\right]_{y}^{2}+d_{*}^{2}\left(\partial_{x} a_{0, \tilde{y}}-\partial_{\tau} a_{1, \tilde{y}}\right)^{2}\right\},
$$

where the nonuniversal length scale $d_{*}$ encodes the flow of $g_{u}$ to strong coupling. Recall that in our formulation, $\vec{a}$ is a noncompact gauge field, and monopoles are included through $\mathcal{L}_{\mathcal{M}}$ [cf. Eq. (38)]. In their absence, bosonic partons would interact logarithmically via the gauge field. (The interaction potential is readily obtained from $\left\langle a_{0} a_{0}\right\rangle$ at $\omega=0$.)

However, it is well known that compact U(1) gauge theories may be unstable to monopole proliferation, i.e., confinement. The relevance of monopoles can be assessed from their correlation function. For an isolated monopoleantimonopole pair at locations $\boldsymbol{R}_{i}=\left(x_{i}, d_{*} y_{i}\right)$ and equal time, it is given by

$$
\begin{aligned}
C_{\boldsymbol{R}_{1}-\boldsymbol{R}_{2}}^{\mathcal{M}} & \equiv\left\langle\mathcal{M}_{y_{1}}^{\dagger}\left(x_{1}\right) \mathcal{M}_{y_{2}}\left(x_{2}\right)\right\rangle \\
& =\frac{\int \mathcal{D}\left[a, \phi_{\mathcal{M}}\right] e^{i \phi_{\mathcal{M}, y_{1}}\left(x_{1}\right)-i \phi_{\mathcal{M}, y_{2}}\left(x_{2}\right)} e^{-\mathcal{S}_{\text {gauge }}-\mathcal{S}_{\mathcal{M}}}}{\int \mathcal{D}\left[a, \phi_{\mathcal{M}}\right] e^{-\mathcal{S}_{\text {gauge }}-\mathcal{S}_{\mathcal{M}}}} .
\end{aligned}
$$

For $g_{\mathcal{M}}=0$, the theory is Gaussian and performing the integral over $\phi_{\mathcal{M}}$ yields

$$
C_{\boldsymbol{R}}^{\mathcal{M}}=\exp \left\{\frac{\kappa d_{*}}{4 \pi^{2} \tilde{v}} \int_{\boldsymbol{k}, \omega} \frac{\cos (\boldsymbol{k} \cdot \boldsymbol{R})-1}{k_{x}^{2}}\left[1-\frac{\kappa d_{*}^{2}}{2 \pi \tilde{v}}\left\langle\left|\varepsilon_{2}\right|^{2}\right\rangle\right]\right\} .
$$

Here $\varepsilon_{2}=\Delta a_{0} / d_{*}$ is the $y$ component of the emergent electric field, $\langle\ldots\rangle$ denotes the Gaussian average over $\vec{a}$, according to $\mathcal{L}_{\mathrm{MW}}$, and $\boldsymbol{k}=\left(k_{x}, k_{y}\right)$. The momentum $k_{y}$ is expressed in units of $d_{*}^{-1}$, i.e., the Fourier transform is defined as $f_{y}(x, \tau)=$ $\frac{\sqrt{d_{*}}}{(2 \pi)^{3 / 2}} \int_{\boldsymbol{k}, \omega} e^{i \boldsymbol{k} \cdot \boldsymbol{R}+i \omega \tau} \tilde{F}(\boldsymbol{k}, \omega)$. Inserting the $\varepsilon_{2}$ correlation function and evaluating the integral for large $|\boldsymbol{R}|$, we find $C_{\boldsymbol{R}}^{\mathcal{M}} \sim$ $\exp \left[\frac{\kappa d_{*}}{2|\boldsymbol{R}|}\right]$.

Since $C_{\boldsymbol{R}}^{\mathcal{M}}$ approaches a nonzero constant at long distances, monopoles are strongly relevant. Beyond a length scale $l_{*}$, we thus expand the cosine in $\mathcal{L}_{\mathcal{M}}$ to quadratic order. Integrating out $\phi_{\mathcal{M}}$ then results in a modified theory for $\vec{a}$ given by

$$
\mathcal{L}_{\text {confining }}=\mathcal{L}_{\mathrm{MW}}+\frac{\kappa}{4 \pi \tilde{v}}\left[\Delta a_{0}\right]_{y} \frac{1}{l_{*}^{2} \partial_{x}^{2}-1}\left[\Delta a_{0}\right]_{y},
$$

with $\mathcal{L}_{\mathrm{MW}}$ as in Eq. (43). The corresponding analytically continued gauge-field propagator has poles at real frequencies $\omega= \pm \tilde{v} \sqrt{k_{x}^{2}+k_{y}^{2}+l_{*}^{-2}}$, i.e., there are no gapless gauge-field modes for finite $l_{*}$.

Alternatively, $C_{\boldsymbol{R}}^{\mathcal{M}}$ can be obtained from $\mathcal{L}_{\mathrm{MW}}$ alone via Eq. (10), i.e., by externally imposing the desired 


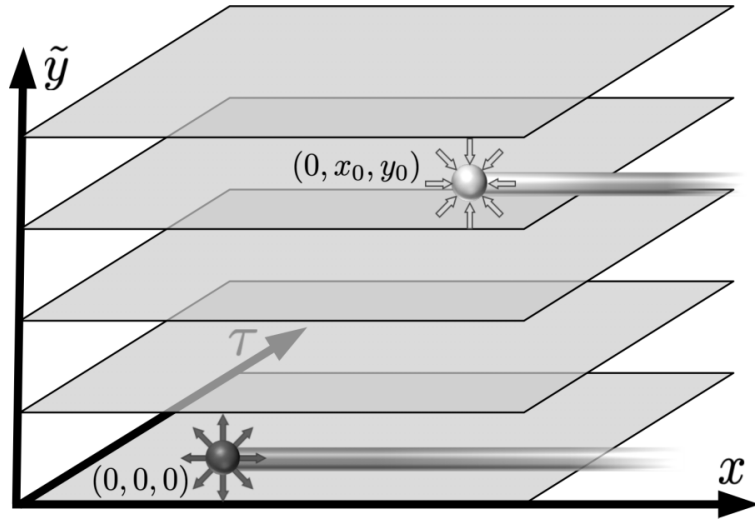

FIG. 7. A singular gauge-field configuration with monopoles of opposite signs can be realized via two flux tubes that extend from infinity at fixed $\tau$ and $y$. Each $x-\tau$ plane represents a dual wire at coordinate $\tilde{y}=y+\frac{1}{2}$, and monopoles are located at $y$, i.e., between wires.

singularities on $\vec{a}$. For specificity, consider $\vec{a}^{\mathcal{M}}$ such that $\left(\partial_{\tau} a_{1}^{\mathcal{M}}-\partial_{x} a_{0}^{\mathcal{M}}\right)=\Delta a_{1}^{\mathcal{M}}=0$ and

$$
\Delta a_{0}^{\mathcal{M}}=2 \pi\left[\delta_{y, 0} H(x)-\delta_{y, y_{0}} H\left(x-x_{0}\right)\right] \delta(\tau) .
$$

This configuration is depicted in Fig. 7. It describes two flux tubes emanating from $x=\infty$ and extending, at fixed $y=0$ and $y_{0}$, to $x=0$ and $x_{0}$, respectively. Inserting this expression into Eq. (10), with the gauge-field action of Eq. (43), we reproduce $C_{\boldsymbol{R}}^{\mathcal{M}}$ in Eq. (45).

Quasiparticles. In the presence of a confining gauge field, individual partons seize to be finite-energy quasiparticles. It would be tempting, but incorrect, to compute their interaction potential directly from the effective gauge theory in Eq. (46). To obtain $\mathcal{L}_{\text {confining }}$, we expanded $g_{\mathcal{M}} \cos \left(2 \phi_{\mathcal{M}}\right)$ around a specific minimum. This restriction to a single topological sector is innocent in the special case of partons on the same dual wire. There, only the trivial sector contributes, and we can indeed use $\mathcal{L}_{\text {confining }}$ to find

$$
V(x)-V(0) \sim \frac{\tilde{v}}{\kappa d_{*}} \int_{k} \frac{1-\cos \left(k_{x} x\right)}{k_{x}^{2}\left(1+l_{*}^{2} k_{y}^{2}\right)} \sim \frac{\tilde{v}}{\beta d_{*} l_{*}}|x| .
$$

In the generic case, a careful sum over different sectors, as performed in Appendix A3, is required. The result is linear confinement in all directions: It is impossible to isolate any excitation charged under the emergent gauge field without incurring a diverging energy cost. Consequently, finite-energy excitations can only be created as combinations of the gauge-neutral quasiparticles $b_{\sigma}^{\dagger} b_{\sigma}$, carrying spin 0 , and $b_{\sigma}^{\dagger} b_{\bar{\sigma}}$ carrying spin 1 .

Spin model. There are two complementary routes to identifying the microscopic phase: through symmetry considerations and by direct translation to a microscopic model. For the former, recall how monopoles transform under the microscopic symmetries (see Table II). The nonzero expectation value acquired by $\mathcal{M}$ implies that $x$-translation symmetry is reduced to translations by two sites. Furthermore, for $g_{\mathcal{M}}<0$, the site-centered $x$ inversion is also broken, while bond-centered inversion is preserved. Other symmetries, in particular time reversal and $U(1)$ spin rotations, remain in- tact. These properties identify the microscopic phase as an intrawire VBS. We arrive at the same conclusion by using the transformation from parton to spin variables. The gauge theory maps onto $\mathcal{L}_{\mathrm{LL}}+\mathcal{L}_{u}$, which we already analyzed in Sec. III B 2. Its gapped ground state exhibits VBS order, $\left\langle\varepsilon_{r}\right\rangle \neq 0$, with integer-spin excitations, exactly as we found in the gauge theory above.

\section{Superfluid and easy-plane AFM}

Mean field. Consider now a superfluid phase where both partons condense. Recall that the parton number is separately conserved for both species. The corresponding U(1) symmetries are spontaneously broken when the tunneling term of Eq. (31a) flows to strong coupling. We proceed as before and expand

$$
\mathcal{L}_{t}=\frac{v_{B} \tilde{g}_{t}}{4 \pi d_{0}^{2}} \cos \left(\varphi_{\tilde{y}+2, \sigma}-\varphi_{\tilde{y}, \sigma}\right) \rightarrow \frac{v_{B}}{8 \pi d_{*}^{2}}\left[\Delta_{2} \varphi_{\sigma}\right]_{\tilde{y}}^{2},
$$

where $\left(\Delta_{2}\right)_{y, y^{\prime}}=\delta_{y+2, y^{\prime}}-\delta_{y, y^{\prime}}$. The field $\theta_{\tilde{y}, \sigma}$ does not enter $\mathcal{L}_{t}$ and can thus be integrated out trivially. Additionally taking the long-wavelength limit $d_{*}^{-1} \Delta_{2} \rightarrow 2 \partial_{y}$, we arrive at the action $\mathcal{S}_{b}^{\mathrm{SF}}=\int_{\tau, x, y} \sum_{\sigma} \mathcal{L}_{\mathrm{SF}, \sigma}$, with

$$
\mathcal{L}_{\mathrm{SF}, \sigma}=\frac{v_{B}}{2 \pi}\left[\frac{1}{c^{2}}\left(\partial_{\tau} \varphi_{\sigma}-a_{0}\right)^{2}+\left(\nabla \varphi_{\sigma}-\boldsymbol{a}\right)^{2}\right] .
$$

This low-energy theory describes two fields that disperse linearly with velocity $c=\sqrt{u_{B} v_{B}}$, Goldstone modes associated with the two condensed parton species. Integrating out $\varphi_{\sigma}$ results in the familiar Meissner response

$$
\mathcal{L}_{\text {Meissner }}=\frac{v_{B}}{2 \pi} \bar{a}_{\mu}\left[\delta^{\mu \nu}-\frac{p^{\mu} p^{\nu}}{\vec{p} \cdot \vec{p}}\right] \bar{a}_{\nu},
$$

where $\vec{p}=\left(\frac{\omega}{c}, k_{x}, k_{y}\right)$ and $\overrightarrow{\vec{a}}=\left(\frac{a_{0}}{c}, a_{1}, a_{2}\right)$. Finally, the periodicity of the original cosine in Eq. (49) permits $2 \pi$ vortices in either condensate, which are logarithmically confined as in Sec. III B 1.

Gauge fluctuations. We reinstate the gauge-field dynamics, governed by $\mathcal{L}_{\mathrm{MW}}+\mathcal{L}_{\mathcal{M}}$ of Eqs. (34) and (38). The induced Meissner term renders the gauge field massive and, thereby, monopoles strongly irrelevant. We verify this explicitly by integrating out $\vec{a}$ to obtain the effective monopole Lagrangian. In the limit of small frequencies and momenta, we find

$$
\mathcal{L}_{\mathcal{M}, \text { eff }}=\frac{\tilde{v}}{4 \pi \kappa}\left[1+\frac{\kappa c^{2} d_{*}^{2}}{2 v_{B} \tilde{v}}\left(\frac{\omega^{2}}{c^{2}}+k_{y}^{2}\right)\right] k_{x}^{2}\left|\phi_{\mathcal{M}}\right|^{2} .
$$

The corresponding monopole-monopole correlation function decays faster than exponentially, i.e.,

$$
C_{\boldsymbol{R}}^{\mathcal{M}} \propto \begin{cases}e^{-x^{2} / \xi_{1}^{2}}, & y=0 \\ e^{-L / \xi_{2}}, & y \neq 0\end{cases}
$$

where $L$ is the wire length, and $\xi_{1,2}$ are nonuniversal length scales.

Quasiparticles. To determine the fate of the partons, we focus on one species and integrate out the other. Recall that the gauge field $\vec{a}$ lives on all dual wires, while each parton species resides on wires with a specific parity. We, therefore, integrate out $b_{\downarrow}$ and the gauge field on even wires to obtain the effective gauge theory for $b_{\uparrow}$ on odd wires. The long-wavelength 


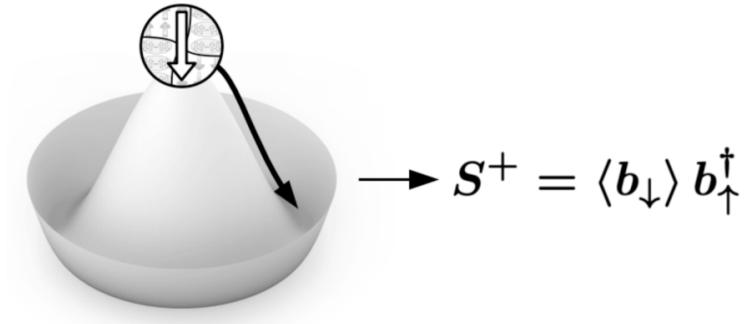

(a) Condensed $b_{\uparrow}$
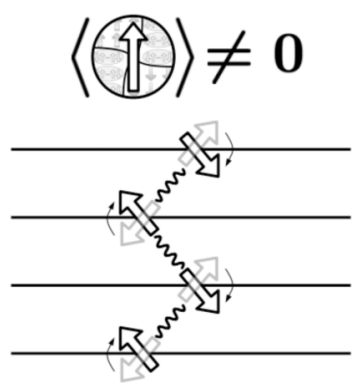

(b) Mott gapped $b_{\uparrow}$

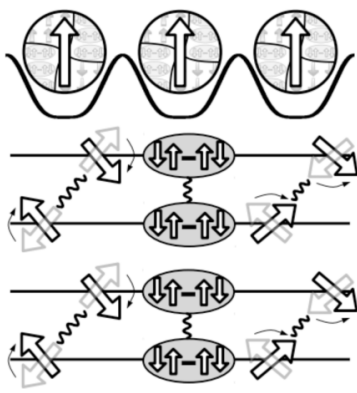

FIG. 8. When one species of bosonic partons condenses, the gauge field acquires a Higgs mass. Moreover, the condensate renders the second parton species local and identifies it with the microscopic spin-raising operator. (a) Condensation of the second species results in a magnetically ordered phase with $\left\langle S^{+}\right\rangle \neq 0$. The corresponding interwire terms are precisely the ones discussed for the easy-plane AFM in Sec. III B 1. (b) When the second parton species forms a Mott insulator, a nonmagnetic state arises. The parton theory that realizes this phase maps onto the coupled-wire model of an interwire VBS.

expansion of the gauge-field action reproduces the form of $\mathcal{L}_{\text {Meissner, }}$ in Eq. (51), with rescaled fields and momenta (see Appendix D 1 for the exact wire-based calculation). Integrating out the massive gauge fluctuations does not qualitatively change the low-energy theory for $b_{\uparrow}$. In the present case, it is of the form $\mathcal{L}_{\mathrm{SF}}$ in Eq. (20), and vortices in the phase of $b_{\uparrow}$ are logarithmically confined.

It is instructive to analyze the role of the external probing field as introduced in Sec. IV A. At the mean-field level, the partons couple to $\vec{A}$ with charges $\left(e_{\uparrow}, e_{\downarrow}\right)=\left(\frac{1}{2},-\frac{1}{2}\right)$. Condensation of either species forces the flux of $\vec{A}$ to be quantized in units of $4 \pi$. However, in the gauge theory, a simple shift $a_{\mu, \tilde{y}} \rightarrow a_{\mu, \tilde{y}}+\frac{1}{4}\left[S A_{\mu}\right]_{\tilde{y}}$ leads to $\left(e_{\uparrow}, e_{\downarrow}\right)=(1,0)$ and, consequently, $2 \pi$ quantization. This apparent ambiguity disappears when gauge fluctuations are accounted for. Indeed, integrating out $\vec{a}$ and $b_{\downarrow}$ in the presence of $\vec{A}$, we find an effective field theory for $b_{\uparrow}$ with $e_{\uparrow}=1$. The same conclusion can be reached by noting that, once $b_{\downarrow}$ has a non-zero expectation value, $b_{\uparrow}$ is identified with the spin raising operator $S^{+}$[see Fig. 8(a)]. Further integrating out the remaining parton $b_{\uparrow}$ yields a Meissner response in the form of Eq. (51) for $\vec{A}$ (see Appendix D 1 for details). Consequently, U(1) spin-rotation symmetry is spontaneously broken.

Spin model. The microscopic phase breaks U(1) spinrotation as well as time-reversal symmetries (see Table II). Moreover, the discrete translation symmetries in the $\hat{x}$ and $\hat{y}$ directions are both reduced to steps of two. These properties identify the microscopic phase as the easy-plane AFM de- scribed in Sec. III B 1. Indeed, the parton gauge theory maps onto $\mathcal{L}_{\mathrm{LL}}+\mathcal{L}_{t}$, which was studied there in detail. We found the same gapless ground state, topological excitations, and, implicitly, the same $2 \pi$ quantization of flux.

\section{Correlated Mott insultor and interwire VBS}

Mean field. Consider now a superfluid phase of one parton species and a Mott insulator of the other, i.e., $\delta \mathcal{L}=g_{t} \cos \left(\varphi_{2 \tilde{y}+2, \downarrow}-\varphi_{2 \tilde{y}, \downarrow}\right)+g_{u} \cos \left(2 \theta_{2 \tilde{y}+1, \uparrow}\right)$. The meanfield analysis is the same as in the two previous cases; the low-energy theory contains the Goldstone mode of the condensed $b_{\downarrow}$ and individual $b_{\uparrow}$ as finite-energy excitation. Vortices in the $b_{\downarrow}$ condensate, created by $V_{\text {odd }} \sim e^{-i \tilde{\varphi}_{\downarrow}}$ [cf. Eq. (30)], are logarithmically confined.

Gauge fluctuations. As in the parton superfluid phase, Sec. IV B 2, the gauge field acquires a Higgs mass through the $b_{\downarrow}$ condensate. Consequently, monopoles can again be safely discarded.

Quasiparticles. The effect of gauge fluctuations on the mean-field excitation $b_{\uparrow}$ can be inferred, as in the last section, by successively integrating out $b_{\downarrow}$ and $\vec{a}$. Exciting a single $b_{\uparrow}$ boson above the Mott gap thus corresponds microscopically to a spin-1 excitation [see Fig. 8(b)]. In addition, vortex excitations $V_{\text {odd }}$ turn into local spin-0 quasiparticles. Formally, this follows from the qualitatively different behavior of the correlation function $\left\langle\left[\Delta_{2} \varphi_{\downarrow}\right]_{2 \tilde{y}+1}\left[\Delta_{2} \varphi_{\downarrow}\right]_{2 \tilde{y}^{\prime}+1}\right\rangle$ at zero frequency: at the mean-field level, it falls off quadratically with distance, but in the gauge theory, it decays exponentially. (For an explicit calculation of the vortex energy, see Appendix A 4.)

Spin model. In this phase, $y$-translation symmetry is broken, but $\tilde{y}$ inversion is preserved (cf. Table II). Moreover, U(1) spin-rotation, time-reversal, and $x$-translation symmetries all remain intact. These symmetry properties, along with the quasiparticle content, imply an interwire VBS. To verify this explicitly, we transcribe the cosines in $\delta \mathcal{L}$ to spin variables

$$
\delta \mathcal{L}_{\text {inter-VBS }}=g_{t} \cos \left([\Delta \Phi]_{2 \tilde{y}+1}\right)+g_{u} \cos \left(2[S \Theta]_{2 \tilde{y}+1}\right) .
$$

One readily verifies that the arguments of the two cosines commute. Consequently, the two terms can simultaneously reach strong coupling. In the resulting fixed-point Hamiltonian, only pairs of wires are coupled. To characterize the ground state, it is thus sufficient to analyze a two-leg ladder.

We diagonalize the interaction by introducing new conjugate variables $\Phi_{ \pm}=\frac{1}{2}\left(\Phi_{2} \pm \Phi_{1}\right)$ and $\Theta_{ \pm}=\left(\Theta_{2} \pm \Theta_{1}\right)$. The fields $\Phi_{-}$and $\Theta_{+}$get trapped around the minima of their respective cosines, and small fluctuations are massive. Fundamental domain walls in the two are created by $D_{+}=e^{i \Phi_{+}}$ and $D_{-}=e^{i \Theta_{-}}$. The former is identified with the spin-raising operator, i.e., $D_{+} \propto S_{1 / 2}^{+}$, with a proportionality factor determined by the pinned $\Phi_{-}$. The latter similarly describes $2 \pi$ phase slips in $S^{+}$, i.e., $D_{-} \propto e^{\mp 2 i \Theta_{1,2}}$. Consequently, the two types of defects carry spin 1 and spin 0 , respectively. Alternatively, the spin can be computed via the general expression

$$
\delta S_{\mathrm{tot}}^{z}[D]=\int_{x} \sum_{y}\left\langle D\left[S_{y}^{z}, D^{\dagger}\right]\right\rangle .
$$

In the present case, $\sum_{y} S_{y}^{z}=\frac{1}{\pi} \partial_{x} \Theta_{+}$and we again find $\delta S_{\text {tot }}^{z}\left[D_{-}\right]=0$ and $\delta S_{\text {tot }}^{z}\left[D_{+}\right]=1$. 


\section{Quantum Hall states and chiral spin liquids}

Mean field. As the first example of a fractionalized phase, consider a bilayer quantum Hall state of bosonic partons. At filling factor $v=2 / n$, it can be realized with the interwire coupling

$$
\delta \mathcal{L}_{\mathrm{QH}}=g_{\mathrm{QH}} \cos \left(\varphi_{\tilde{y}+1, \sigma}-\varphi_{\tilde{y}-1, \sigma}-2 n \theta_{\tilde{y}, \bar{\sigma}}\right) .
$$

This wire construction was proposed in Ref. [51] for microscopic bosons $e^{i \varphi}$ and analyzed in detail. Adapted to the present context, the resulting phase hosts two species of spin- $1 / 2 n$ excitations that are self-bosons but exhibit mutual statistics $\pi / n$, i.e., the corresponding $K$ matrix is $n$ times the Pauli matrix $\sigma_{x}$. To find $K$, we calculate the response to $\vec{a}$ by replacing $\delta \mathcal{L}_{\mathrm{QH}}$ with its quadratic expansion and integrating out the matter fields (see Appendix D2). The leading contribution at long wavelength is

$$
\mathcal{L}_{\mathrm{CS}}=\frac{i}{2 \pi n}\left[S a_{1}\right]_{y}\left[\Delta a_{0}\right]_{y} \sim \frac{i}{2 \pi n} \epsilon_{\mu \nu} a_{\mu} \partial_{y} a_{\nu},
$$

where $\epsilon_{\mu \nu}$ is the antisymmetric tensor. Endowing $\vec{a}$ with a (redundant) spin label according to the dual-wire parity, the induced action for $\vec{a}_{\sigma}$ takes the expected form. In the continuum limit, we find

$$
\mathcal{S}_{\mathrm{CS}}=\frac{i}{4 \pi} \int_{x, y, \tau} \sum_{\sigma, \sigma^{\prime}} \epsilon_{\mu \nu \kappa} a_{\mu, \sigma}\left[K^{-1}\right]_{\sigma \sigma^{\prime}} \partial_{\nu} a_{\kappa, \sigma^{\prime}}
$$

in the gauge $a_{2}=0$ and with $K=n \sigma_{x}$.

Gauge fluctuations. We restore the status of $\vec{a}$ as dynamical, with fluctuations governed by the sum of the induced ChernSimons and bare Maxwell terms. The latter contains the contribution $\propto\left[\Delta a_{\mu}\right]^{2}$, which translates into $\propto\left(a_{\mu, \uparrow}-a_{\mu, \downarrow}\right)^{2}$. This term renders the antisymmetric combination of $a_{\mu, \sigma}$ massive, while the Chern-Simons term results in a gap for the symmetric combination. Consequently, monopoles are strongly irrelevant and can be safely discarded.

The Chern-Simons action, Eq. (57), implies that both microscopic and bosonic-parton time-reversal symmetries are broken. To see the latter, it is convenient to compute the response to the external probing field $\vec{A}$. Including it amounts to replacing $a_{\mu, \tilde{y}} \rightarrow a_{\mu, \tilde{y}}-\frac{1}{4}(-1)^{\tilde{y}}\left[S A_{\mu}\right]_{\tilde{y}}$ in the induced action (but not in the bare one). Integrating out the emergent gauge field we find the response

$$
\mathcal{L}_{A-\mathrm{CS}}=-\frac{i}{8 \pi n}\left[S A_{1}\right]_{\tilde{y}}\left[\Delta A_{0}\right]_{\tilde{y}} \sim-\frac{i}{8 \pi n} \epsilon_{\mu \nu} A_{\mu} \partial_{y} A_{\nu} .
$$

Consequently, these phases are chiral and must exhibit topologically protected edge states.

Quasiparticles. To identify the quasiparticles, we transform the partons into new composite bosons. Specifically, we attach to each parton $n$ fluxes of the opposite one. On the operator level, this procedure amounts to introducing bosons $\beta_{\tilde{y}, \sigma}=e^{-i \eta_{\tilde{y}, \sigma}}$ with $\eta_{\tilde{y}, \sigma} \equiv \varphi_{\tilde{y}, \sigma}+n \tilde{\varphi}_{\bar{\sigma}, \tilde{y}}$. Such manipulations often become more transparent in a schematic description that specifies only the couplings between particle currents $\vec{j}_{b, \sigma}$ and gauge fields. The bosonic-parton theory in Eqs. (33) and (34) is then expressed as

$$
\mathcal{L}_{b}=i \sum_{\sigma} \vec{j}_{b, \sigma} \cdot\left(\vec{a}+\frac{\sigma}{2} \vec{A}\right)+\cdots,
$$

where the ellipsis denotes kinetic terms for partons and dynamical gauge fields as well as short-range interactions $(\vec{A}$, as always, is an external probing field). Attaching $n$ mutual fluxes amounts to replacing $\mathcal{L}_{b} \rightarrow \mathcal{L}_{\beta}$ with

$$
\mathcal{L}_{\beta}=i \sum_{\sigma} \vec{j}_{\beta, \sigma} \cdot\left(\vec{c}_{\sigma}+\vec{a}+\frac{\sigma}{2} \vec{A}\right)-\frac{i}{2 \pi n} c_{\uparrow} d c_{\downarrow} .
$$

Finally, we shift $\vec{c}_{\sigma} \rightarrow \vec{c}_{\sigma}-\vec{a}$ to decouple $\vec{a}$ from the matter fields and integrate it out to obtain

$$
\mathcal{L}_{\beta}^{\prime}=i \sum_{\sigma} \vec{j}_{\beta, \sigma} \cdot\left(\vec{c}_{\sigma}+\frac{\sigma}{2} \vec{A}\right)+i \frac{\left(c_{\uparrow}-c_{\downarrow}\right) d\left(c_{\uparrow}-c_{\downarrow}\right)}{8 \pi n} .
$$

In terms of composite bosons, the interwire coupling reads as $\delta \mathcal{L}_{\mathrm{QH}}=g_{\mathrm{QH}} \cos \left(\eta_{\tilde{y}+1, \sigma}-\eta_{\tilde{y}-1, \sigma}\right)$, i.e., $\beta_{\sigma}$ form a superfluid. At the mean-field level, the excitations are two flavors of logarithmically confined vortices in the phases of $\beta_{\sigma}$. In the presence of the dynamical gauge fields $\vec{c}_{\sigma}$, these turn into finite-energy excitations subject to the constraint that $(-2 \pi)$ flux of $\vec{c}_{\sigma}$ must be accompanied by $\sigma / 2 n$ charge of each boson, i.e.,

$$
\rho_{\sigma}^{\beta}=-\frac{1}{4 \pi n} \vec{\nabla} \times \vec{c}_{\sigma}+\frac{1}{4 \pi n} \vec{\nabla} \times \vec{c}_{\bar{\sigma}} .
$$

Since $\rho_{\sigma}^{\beta}$ is related to the physical spin [charge under $\vec{A}$, see Eq. (62)] via $S^{z}=\left(\rho_{\uparrow}^{\beta}-\rho_{\downarrow}^{\beta}\right) / 2$, the composites carry a total spin of $1 / 2 n$. Moreover, a clockwise exchange results in a statistical phase $\pi / 2 n$.

Spin model. The response to the external probing field, Eq. (59), implies that the microscopic phase is a chiral QSL with topological edge states and fractionalized quasiparticles in the bulk. Translating the interwire coupling in Eq. (56) to microscopic variables we find

$$
\delta \mathcal{L}_{\mathrm{CSL}}=g_{\mathrm{QH}} \cos \left([\Delta \Phi]_{\tilde{y}}+2 n[S \Theta]_{\tilde{y}}\right) .
$$

Precisely this coupling, with $n=1$, was proposed in Refs. [82,83], where it was shown to realize the Kalmeyer-Laughlin chiral spin liquid; the generalization to arbitrary integers $n$ is straightforward. (The same coupling term also describes a bosonic Laughlin state at filling factor $v=1 / 2 n$, see Ref. [51].) In particular, bulk quasiparticles carry spin $1 / 2 n$ and acquire phases $\pi / 2 n$ upon (clockwise) exchange.

\section{Pair condensate $/ \mathbb{Z}_{2}$ spin liquid}

As the final example with bosonic partons, we construct a time-reversal-invariant gapped QSL. Here, the emergent gauge field must acquire a Higgs mass without condensation of either of the two species (which would lead to a symmetrybroken phase as discussed in Secs. IV B 2 and IV B 3). These requirements are satisfied when composites with higher emergent gauge charges, such as parton pairs, condense.

Mean field. In the coupled-wire framework, realizing such a phase is straightforward. To form a superfluid of parton pairs $\Psi_{2 y} \equiv b_{2 \tilde{y}, \downarrow} b_{2 \tilde{y}-1, \uparrow} \equiv e^{i 2 \varphi_{2 y}^{+}}$, we introduce the interwire coupling

$$
\delta \mathcal{L}_{\text {pair }}=g_{p} \Psi_{2 y+2}^{\dagger} \Psi_{2 y}+\text { H.c. }=g_{p} \cos \left(2\left[\Delta_{2} \varphi^{+}\right]_{2 y+1}\right) .
$$


Once this term flows to strong coupling, $\Psi$ spontaneously acquires an expectation value (cf. Sec. IV B 2). Individual partons, however, are not condensed. To describe their properties, we introduce $\varepsilon_{2 y}=e^{-i \varphi_{2 y}^{-}}$with $\varphi_{2 y}^{-} \equiv\left(\varphi_{2 \tilde{y}, \downarrow}-\varphi_{2 \tilde{y}-1, \uparrow}\right) / 2$. The expectation value acquired by the pair field relates $\varepsilon_{2 y} \propto$ $b_{2 \tilde{y}, \downarrow} \propto b_{2 \tilde{y}-1, \uparrow}^{\dagger}$. A gapped phase where neither $b_{\sigma}$ condense is realized by including the interaction

$$
\delta \mathcal{L}_{\text {Mott } \varepsilon}=g_{q} \cos \left(2 \theta_{2 y}^{-}\right)=g_{q} \cos \left(2 \theta_{2 \tilde{y}, \downarrow}-2 \theta_{2 \tilde{y}-1, \uparrow}\right) .
$$

Elementary domain walls in this cosine are created by individual partons, which constitute the fundamental quasiparticles at the mean-field level. Vortices in the pair condensate, created by $m_{2 y+1}^{\dagger} \equiv e^{i\left(\tilde{\varphi}_{\uparrow, 2 \tilde{y}+1}+\tilde{\varphi}_{\downarrow, 2 \tilde{y}}\right) / 2}$, are logarithmically confined.

Gauge fluctuations. We reinstate the gauge-field dynamics and integrate out the matter fields. The pair condensate leads to a Higgs mass for the emergent gauge field $\vec{a}$ as in Sec. IV B 2. Consequently, monopoles are again strongly irrelevant and can be safely discarded.

Quasiparticles. The analysis of quasiparticle excitations closely mirrors the one in Sec. IV B 3. Integrating out both $\theta^{+}, \varphi^{+}$and the gauge field $\vec{a}$ results, to lowest order in $\Delta$, in

$$
\begin{aligned}
\mathcal{L}_{\varepsilon}^{\mathrm{eff}}= & \frac{i}{\pi} \partial_{x} \theta_{2 y}^{-}\left(\partial_{\tau} \varphi_{2 y}^{-}+\frac{1}{2} A_{0,2 y}\right)+\frac{\bar{v}}{2 \pi}\left(\partial_{x} \varphi_{2 y}^{-}+\frac{1}{2} A_{1,2 y}\right)^{2} \\
& +\frac{\bar{u}}{2 \pi}\left(\partial_{x} \theta_{2 y}^{-}\right)^{2}+g_{q} \cos \left(2 \theta_{2 y}^{-}\right),
\end{aligned}
$$

with renormalized parameters $\bar{v}$ and $\bar{u}$. Consequently, $\varepsilon \sim$ $\left\langle b_{\uparrow}^{\dagger}\right\rangle_{\varphi^{+}, \vec{a}} \sim\left\langle b_{\downarrow}\right\rangle_{\varphi^{+}, \vec{a}}$ creates a deconfined bosonic spin- $\frac{1}{2}$ excitation, as in the mean-field discussion. In addition, the dynamical gauge field liberates vortices $m$ from their logarithmic confinement as in Sec. IV B 3; they become bona fide spin-0 bosonic quasiparticles.

To infer the mutual statistics between the two quasiparticles, consider the hopping of $\varepsilon$. It stems from the microscopic term

$$
N_{2 y+1}^{+} N_{2 y}^{-}=e^{i \varphi_{2 y+2}^{+}-i \varphi_{2 y}^{+}} \varepsilon_{2 y}^{\dagger} \varepsilon_{2 y+2} \equiv t_{2 y, 2 y+2} \varepsilon_{2 y}^{\dagger} \varepsilon_{2 y+2},
$$

i.e., the hopping amplitude is set by the pair condensate. In the absence of vortices, $t$ is uniform. For a static vortex-antivortex pair, there is instead a branch cut connecting the two, across which the phase of $t$ jumps by $\pi$ (see Fig. 9). For dynamical quasiparticles $m$ and $\varepsilon$, this property implies mutual semionic statistics.

Spin model. The quantum numbers and braiding properties of quasiparticles are characteristic of a $\mathbb{Z}_{2}$ QSL. Time-reversal symmetry is preserved in this phase, but translation symmetry in the $\hat{y}$ direction is reduced to translations by two wires. To analyze this phase in terms of microscopic spin variables, we translate the intrawire couplings of Eqs. (65) and (66), finding

$$
\begin{aligned}
\delta \mathcal{L}_{\mathbb{Z}_{2}}= & g_{p} \cos \left(\Phi_{2 y+2}-2 \Phi_{2 y+1}+\Phi_{2 y}\right) \\
& +g_{q} \cos \left(2 \Theta_{2 y+1}+4 \Theta_{2 y}+2 \Theta_{2 y-1}\right) .
\end{aligned}
$$

The two cosines do not compete, and their arguments can thus be pinned simultaneously. For an even number of wires with periodic boundary conditions in the $\hat{y}$ direction, there are as many linearly independent pinned fields as there are wires. Consequently, a fully gapped phase can form.

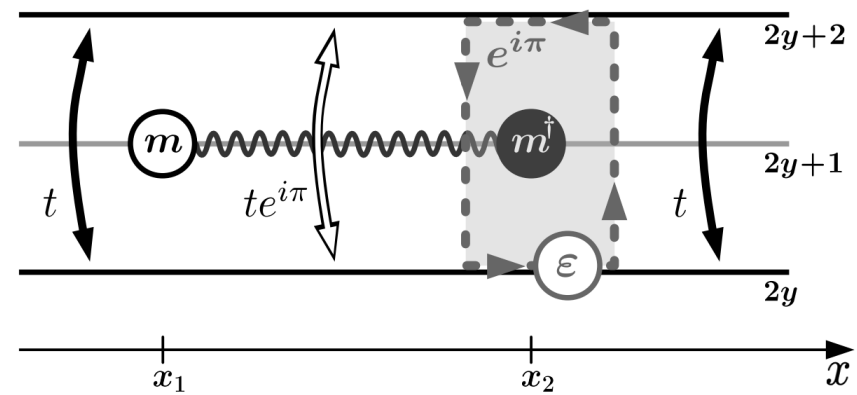

FIG. 9. In the parton-pair condensate, a static configuration of $m$ quasiparticles modifies the hopping amplitudes of $\varepsilon$ excitations. An $m$ quasiparticle-quasihole pair at $x_{1}$ and $x_{2}$ is connected by a branch cut (wavy line), across which the phase of $\varepsilon$ hopping changes by $\pi$. Consequently, the $\varepsilon$ quasiparticle acquires a minus sign upon encircling $m$, i.e., the two are mutual semions.

The microscopically allowed operator $e^{i 2 \Theta_{2 y}}$ increments the arguments of two adjacent $g_{p}$ cosines by $2 \pi$, i.e., it creates a pair of fundamental domain walls. Similarly, $e^{-i 2 \Theta_{2 y+1}}$ creates a strength-2 domain wall in a single $g_{p}$ cosine. Consequently, a domain wall and an antidomain wall on the wires $2 y_{2}+1$ and $2 y_{1}-1$ are created by

$$
\begin{aligned}
\mathcal{O}_{2 y_{1}-1,2 y_{2}+1}^{m} & \equiv \exp \left[-2 i \sum_{2 y_{1} \leqslant y \leqslant 2 y_{2}} \Theta_{y}\right] \\
& =\exp \left[i \Theta_{2 y_{1}-1}\right] \exp \left[-i \Theta_{2 y_{2}+1}\right] s_{2 y_{1}, 2 y_{2}}^{m} .
\end{aligned}
$$

The string operator $s_{2 y_{1}, 2 y_{2}}^{m}$ is comprised solely of the pinned combinations $\Theta_{2 y+1}+2 \Theta_{2 y}+\Theta_{2 y-1}$ and acquires a nonzero expectation value. Domain walls are thus deconfined in the $\hat{y}$ direction. Additionally, one readily verifies that $\mathcal{O}_{2 y_{1}-1,2 y_{2}+1}^{m} \propto m_{2 y_{2}-1}^{\dagger} m_{2 y_{1}+1}$ in the ground state. To move the domain wall along the wire direction, one need only apply $\mathcal{O}_{2 y_{0}-1}^{m}\left(x_{1}, x_{2}\right)=e^{-i \int_{x_{1}}^{x_{2}} d x \partial_{x} \Theta_{2 y_{0}-1}}$. These domain-wall excitations are thus precisely the $m$ quasiparticles discussed in the gauge-theory analysis.

Similarly, we construct a second species of deconfined excitations via the operator $e^{i(-1)^{y} \Phi_{y}}$. A quasiparticle-quasihole pair on wires $2 y_{2}$ and $2 y_{1}$ is created by

$$
\begin{aligned}
\mathcal{O}_{2 y_{1}, 2 y_{2}}^{\varepsilon} & \equiv \exp \left[i \sum_{2 y_{1}<y \leqslant 2 y_{2}}(-1)^{y} \Phi_{y}\right] \\
& =\exp \left[-\frac{i}{2} \Phi_{2 y_{1}}\right] \exp \left[\frac{i}{2} \Phi_{2 y_{2}}\right] s_{2 y_{1}+1,2 y_{2}-1}^{\varepsilon} .
\end{aligned}
$$

The string operator $s_{2 y_{1}+1,2 y_{2}-1}^{\varepsilon}$ is also expressible in terms of pinned fields only, specifically the combination $\Phi_{2 y+2}-2 \Phi_{2 y+1}+\Phi_{2 y}$. Consequently, $\mathcal{O}_{2 y_{1}, 2 y_{2}}^{\varepsilon} \propto \varepsilon_{2 y_{2}}^{\dagger} \varepsilon_{2 y_{1}}$ in the ground state and $\varepsilon$ is deconfined in the $\hat{y}$ direction. Finally, $\mathcal{O}_{2 y_{0}}^{\varepsilon}\left(x_{1}, x_{2}\right)=e^{\frac{i}{2} \int_{x_{1}}^{x_{2}} d x \partial_{x} \Phi_{2 y_{0}}}$ moves $\varepsilon$ along $\hat{x}$.

The spin of $m$ and $\varepsilon$ can be inferred from the operators that terminate the strings in $\mathcal{O}^{m}$ and $\mathcal{O}^{\varepsilon}$ using Eq. (55). The $m$ quasiparticle is spinless while $\varepsilon$ carries spin $\frac{1}{2}$. Finally, we compute the exchange statistics of the quasiparticles. Since all terms in Eq. (70) commute, $m$ has trivial self-statistics, i.e., it is a boson. The same holds for $\varepsilon$. Their mutual statistics can 


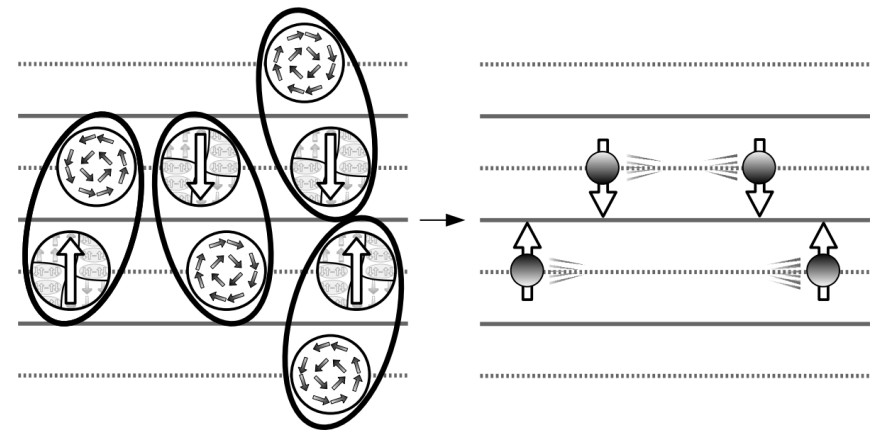

FIG. 10. Magnetic vortices and dislocations are dual to each other, similar to bosons and vortices in a superfluid. Combinations of the two defects, therefore, exhibit fermionic statistics. These composite fermions inherit their species from the dislocation, i.e., $\sigma=\uparrow$ or $\sigma=\downarrow$ depending on the dual-wire parity. The relative orientation of magnetic vortex and dislocation determines the chirality of the fermion, as in the coupled-wire implementation of conventional flux attachment [96].

be read off from

$$
e^{i 2 \alpha}=U_{1} U_{2} U_{1}^{\dagger} U_{2}^{\dagger},
$$

where $U_{1}=\mathcal{O}_{2 y_{0}-1}^{m}\left(x_{1}, x_{2}\right)$ and $U_{2}=\mathcal{O}_{2 y_{1}, 2 y_{2}}^{\varepsilon}\left(x_{0}\right)$. Braiding occurs when the paths of $\varepsilon$ and $m$ interlink, i.e., for $y_{0} \in$ $\left[y_{1}, y_{2}\right]$ and $x_{0} \in\left[x_{1}, x_{2}\right]$. In that case, we find $e^{i 2 \alpha}=-1$, which implies that the two quasiparticles are mutual semions. Consequently, they can combine to form a fermion, schematically $\mathcal{O}^{\psi} \sim \mathcal{O}^{\varepsilon} \mathcal{O}^{m}$. We will see that these fermions exactly coincide with the partons that are the focus of the next section.

\section{FERMIONIC PARTONS FROM COUPLED WIRES}

Above, we have seen that the topological defects in a VBS form a bosonic-parton representation of spins. We now show that fermionic partons can also be constructed from topological defects, specifically as composites of magnetic vortices and dislocations. This route to fermionic partons is closely related to the well-known flux attachment [112,113] (see also Refs. [102,114-116] for recent refinements). Recall that $b_{\uparrow}^{\dagger}$ is dual to one flavor of magnetic vortices $V_{\uparrow}=e^{i \tilde{\varphi}_{\uparrow}}$, while $b_{\downarrow}$ is dual to the other $V_{\downarrow}=e^{i \tilde{\varphi}_{\downarrow}}$ [see discussion near Eq. (30)]. Therefore, fermions can be constructed as $f_{\sigma}=b_{\sigma} V_{\sigma}$, i.e., by attaching $2 \pi \sigma$ flux to the bosonic partons (see Fig. 10). Schematically this transformation can be expressed as

$$
i \sum_{\sigma} \vec{j}_{b, \sigma} \cdot \vec{a} \rightarrow i \sum_{\sigma} \vec{j}_{f, \sigma} \cdot\left(\vec{c}_{\sigma}+\vec{a}\right)+\frac{\sigma}{4 \pi} c_{\sigma} d c_{\sigma} .
$$

Performing the shift $c_{\mu, \sigma} \rightarrow c_{\mu, \sigma}-a_{\mu}$ and integrating out $a_{\mu}$ yields the constraint $\vec{c}_{\uparrow}=\vec{c}_{\downarrow} \equiv \vec{c}$. The resulting theory has the same structure as the bosonic one: two species of fermions that are minimally coupled to an emergent gauge field $\vec{c}$, which obeys Maxwell dynamics.

To implement these manipulations in the wire array, we introduce new variables

$$
\begin{aligned}
& \varphi_{f, \tilde{y}, \sigma}=\varphi_{\tilde{y}, \sigma}+\frac{\sigma}{2}\left(\tilde{\varphi}_{\sigma, \tilde{y}+1}+\tilde{\varphi}_{\sigma, \tilde{y}-1}\right), \\
& \theta_{f, \tilde{y}, \sigma}=\theta_{\tilde{y}, \sigma}
\end{aligned}
$$

where $\tilde{\varphi}_{\sigma}$ was defined in Eq. (30). The linear combinations $\phi_{\sigma}^{\chi}=\varphi_{\sigma}+\chi \theta_{\sigma}$, with $\chi=R / L=+/-$, satisfy

$$
\begin{aligned}
{\left[\phi_{\tilde{y}, \sigma}^{\chi}(x), \phi_{\tilde{y}^{\prime}, \sigma^{\prime}}^{\chi^{\prime}}\left(x^{\prime}\right)\right]=} & i \pi \delta_{\sigma \sigma^{\prime}}\left\{\chi \delta_{\chi \chi^{\prime}} \delta_{\tilde{y} \tilde{y}^{\prime}} \operatorname{sgn}\left(x-x^{\prime}\right)\right. \\
& \left.+\sigma \operatorname{sgn}\left(\tilde{y}^{\prime}-\tilde{y}\right)+\delta_{\tilde{y} \tilde{y}^{\prime}} \epsilon_{\chi \chi^{\prime}}\right\},
\end{aligned}
$$

with the convention that $\operatorname{sgn}(0)=0$. These commutators imply that the operators $f_{\tilde{y}, \sigma, \chi}^{\dagger} \sim e^{i \phi_{\tilde{y}, \sigma}^{x}}$ anticommute for equal $\sigma$. The associated densities $\rho_{\tilde{y}, \sigma, \chi} \equiv \frac{\chi}{2 \pi} \partial_{x} \phi_{\tilde{y}, \sigma, \chi}$ are chiral; they describe right and left movers for $\chi=R$ and $L$, respectively. Moreover, the total density on the $\tilde{y}$ th dual wire, $\rho_{\tilde{y}, \sigma}=$ $\rho_{\tilde{y}, \sigma, R}+\rho_{\tilde{y}, \sigma, L}=\frac{1}{\pi} \partial_{x} \theta_{f, \tilde{y}, \sigma}$, is identical to that of bosonic partons. Consequently, the particle number of each species $Q_{\sigma}$ is separately conserved [cf. Eq. (29)] and particles created by $f_{\uparrow}$ and $f_{\downarrow}$ are distinguishable. Their exchange phase, which is trivial according to Eq. (75), is merely a gauge choice. ${ }^{3}$ Only phases acquired during full braiding processes carry significance; in the present case, they are also trivial. These properties identify $f_{\sigma}$ with the fermions obtained via the schematic flux attachment described by Eq. (73).

To translate generic interwire couplings, the following identities are useful:

$$
\begin{aligned}
e^{i\left(2 \Theta_{y+1}+2 \Theta_{y}\right)} & = \begin{cases}f_{\tilde{y}, \downarrow, L}^{\dagger} f_{\tilde{y}, \downarrow, R}, & \tilde{y} \text { even } \\
f_{\tilde{y}, \uparrow, R}^{\dagger} f_{\tilde{y}, \uparrow, L}, & \tilde{y} \text { odd }\end{cases} \\
e^{i\left(\Phi_{y+1}-\Phi_{y}\right)} & = \begin{cases}f_{\tilde{y}+1, \uparrow, R}^{\dagger} f_{\tilde{y}-1, \uparrow, L}, & \tilde{y} \text { even } \\
f_{\tilde{y}-1, \downarrow, R}^{\dagger} f_{\tilde{y}+1, \downarrow, L}, & \tilde{y} \text { odd. }\end{cases}
\end{aligned}
$$

In particular, the interwire couplings in Eq. (18), which generate the AFM and VBS phases, become simple hopping terms for the fermions

$$
\begin{aligned}
\sum_{y} \mathcal{L}_{t} & =g_{t} \sum_{\tilde{y}} f_{\tilde{y}+\sigma, \sigma, R}^{\dagger} f_{\tilde{y}-\sigma, \sigma, L}+\text { H.c. }, \\
\sum_{y} \mathcal{L}_{u} & =g_{u} \sum_{\tilde{y}} f_{\tilde{y}, \sigma, R}^{\dagger} f_{\tilde{y}, \sigma, L}+\text { H.c. }
\end{aligned}
$$

As in the case of bosonic partons, trivial umklapp processes are allowed, which implies unit filling. When it is the most relevant term, $\mathcal{L}_{u}$ opens a band gap, as would be the case for weakly interacting fermions. In the present case, such a trivial phase can be avoided by several mechanisms: First, it stands in competition with a quantum Hall insulator generated by $\mathcal{L}_{u}$. Second, interactions can render correlated processes strongly relevant and drive the partons to a new fixed point where umklapp processes are irrelevant. Lastly, in certain microscopic spin models $\mathcal{L}_{u}$, is altogether absent. This is the case, e.g., on a triangular lattice due to geometric frustration [117].

Finally, destroying a parton of spin $\downarrow$ and creating one with spin $\uparrow$ yields the smooth component of the microscopic spinraising operators

$$
\begin{aligned}
& f_{2 \tilde{y}+1, \uparrow, R}^{\dagger} f_{2 \tilde{y} \downarrow, R}=J_{2 y+1, R}^{+} \equiv e^{i \Phi_{2 y+1}+2 i \Theta_{2 y+1}}, \\
& f_{2 \tilde{y}-1, \uparrow, L}^{\dagger} f_{2 \tilde{y}, \downarrow, L}=J_{2 y, L}^{+} \equiv e^{i \Phi_{2 y}-2 i \Theta_{2 y}},
\end{aligned}
$$

\footnotetext{
${ }^{3} \mathrm{~A}$ redefinition $f_{\uparrow} \rightarrow f_{\uparrow} e^{i \pi Q_{\downarrow}}$ results in the anticommutation of $f_{\uparrow}$ and $f_{\downarrow}$ without affecting the action or the full-braiding phase.
} 
similar to the parton decomposition of Eq. (3). The analogous expression for bosonic partons instead gives the staggered component of the spin. Of course, both contributions must be encoded in either parton representation. The respective missing ones are encoded nonlocally in monopole operators, as we will see below.

\section{A. Gauge theory}

To derive the action for fermionic partons, we proceed as we did for the bosons in Sec. IV A. We find $\mathcal{S}_{f}=$ $\int_{x, \tau} \sum_{\tilde{y}}\left[\mathcal{L}_{f}+\mathcal{L}_{\text {Maxwell }}+\mathcal{L}_{\text {int }}\right]$ with

$$
\begin{aligned}
\mathcal{L}_{f}= & \frac{i}{\pi} \partial_{x} \theta_{f, \tilde{y}, \sigma}\left(\partial_{\tau} \varphi_{f, \tilde{y}, \sigma}-a_{0, \tilde{y}}\right) \\
& +\frac{v_{F}}{2 \pi}\left(\partial_{x} \varphi_{f, \tilde{y}, \sigma}-a_{1, \tilde{y}}\right)^{2}+\frac{u_{F}}{2 \pi}\left(\partial_{x} \theta_{f, \tilde{y}, \sigma}\right)^{2}, \\
\mathcal{L}_{\text {Maxwell }}= & \frac{\kappa}{8 \pi \tilde{v}}\left[\Delta a_{0}\right]_{y}^{2}+\frac{\kappa \tilde{v}}{8 \pi}\left[\Delta a_{1}\right]_{y}^{2} .
\end{aligned}
$$

The final term $\mathcal{L}_{\text {int }}$ contains exponentially decaying inter-wire terms (see Appendix $\mathrm{C}$ for a detailed derivation and expressions for $v_{F}, \kappa$, and $\tilde{v}$ ). It is instructive to express $\mathcal{L}_{f}$ in terms of the nonchiral fermions $f_{\tilde{y}, \sigma}=f_{\tilde{y}, \sigma, R} e^{i k_{F} x}+f_{\tilde{y}, \sigma, L} e^{-i k_{F} x}$. We find

$$
\mathcal{L}_{f}^{\prime}=f_{\tilde{y}, \sigma}^{\dagger}\left(\partial_{\tau}-i a_{0}-\mu\right) f_{\tilde{y}, \sigma}+\frac{v_{F}}{2 k_{F}}\left|\left(\partial_{x}-i a_{1}\right) f_{\tilde{y}, \sigma}\right|^{2} .
$$

To determine the chemical potential $\mu$, notice that the value of $k_{F}$ only carries significance relative to another length. In the present case, this scale is given by the lattice spacing of the underlying spin chain, which enters the fermionic theory through Eq. (77).

\section{Monopoles}

The $4 \pi$ phase-slip term of Eq. (13) is nonlocal in terms of fermionic partons. In the discussion of bosonic partons, we expressed phase slips through the operator $\widehat{\mathcal{M}}$ [cf. Eq. (36)]. Since $\theta_{\sigma}=\theta_{f, \sigma}$, it again acts as the insertion of a fundamental $(2 \pi)$ monopole in the emergent gauge field $\vec{a}$. Microscopically, monopoles encode the Néel vector through $N_{y}^{+}=$ $\widehat{\mathcal{M}}_{y} J_{y, \chi}^{+}$, with $\chi=(-1)^{y}$, and where $J^{+}$is expressed using fermion operators in Eq. (78). The same procedure as for bosonic partons (cf. Sec. IV A 1) leads to the monopole Lagrangian

$$
\mathcal{L}_{\mathcal{M}}=\frac{g_{\mathcal{M}}}{2}\left[\mathcal{M}_{\boldsymbol{r}}^{2}+\mathcal{M}_{\boldsymbol{r}}^{2 \dagger}\right]-\frac{\kappa}{8 \pi \tilde{v}}\left[\Delta a_{0}-\frac{i 2 \tilde{v}}{\kappa} \partial_{x} \phi_{\mathcal{M}}\right]_{y}^{2},
$$

with parameters $\kappa, \tilde{v}$ as in Eq. (80). As before, when $g_{\mathcal{M}}=$ 0 , the monopole field $\phi_{\mathcal{M}}$ does not affect any gauge-field or matter correlation function.

\section{Symmetries}

We conclude the description of the parton gauge theory by discussing how microscopic symmetries are encoded. One significant difference from the case of bosonic partons is that certain microscopic symmetries are realized nonlocally. This property may be readily understood from the flux-attachment interpretation of fermionic partons: Time-reversal flips the
TABLE III. The action of microscopic symmetries on the fermionic partons, and their gauge-theory interpretation. Certain symmetries such as time reversal act nonlocally as dualities, i.e., transform $f_{\sigma}$ into $d_{\sigma}$. As before, asterisks denote antiunitary symmetries.

\begin{tabular}{lrcl}
\hline \hline $\begin{array}{l}\text { Microscopic } \\
\text { symmetry }\end{array}$ & $f_{\uparrow, R}^{\dagger} f_{\downarrow, L}$ & $f_{\sigma, R}^{\dagger} f_{\sigma, L}$ & $\begin{array}{l}\text { Parton } \\
\text { interpretation }\end{array}$ \\
\hline$U(\alpha)$ & $e^{i \alpha} f_{\uparrow, R}^{\dagger} f_{\downarrow, L}$ & $f_{\sigma, R}^{\dagger} f_{\sigma, L}$ & Global $Q_{\uparrow}-Q_{\downarrow}$ \\
& & & gauge transform. \\
$\mathcal{T}^{*}$ & $-d_{\uparrow, L}^{\dagger} d_{\downarrow, R}$ & $d_{\sigma, L}^{\dagger} d_{\sigma, R}$ & Duality \\
$\Pi_{y}$ & $-f_{\downarrow, L}^{\dagger} f_{\uparrow, R}$ & $f_{\sigma, L}^{\dagger} f_{\sigma, R}$ & PH \\
$T_{x}$ & $f_{\uparrow, R}^{\dagger} f_{\downarrow, L}$ & $f_{\sigma, R}^{\dagger} f_{\sigma, L}$ & $x$ translation \\
$T_{y}$ & $d_{\downarrow, R}^{\dagger} d_{\uparrow, L}$ & $d_{\bar{\sigma}, R}^{\dagger} d_{\bar{\sigma}, L}$ & TR + duality \\
$\mathcal{T}^{*}$ & $f_{\downarrow, L}^{\dagger} f_{\uparrow, R}$ & $f_{\bar{\sigma}, L}^{\dagger} f_{\bar{\sigma}, R}$ & TR \\
$\Pi_{y} T_{y} \mathcal{I}_{x}$ & $f_{\downarrow, L}^{\dagger} f_{\uparrow, R}$ & $f_{\bar{\sigma}, L}^{\dagger} f_{\bar{\sigma}, R}$ & $x$ inv. + spin flip \\
$\Pi_{y} T_{y} \mathcal{I}_{y}$ & $-f_{\downarrow, R}^{\dagger} f_{\uparrow, L}$ & $f_{\bar{\sigma}, R}^{\dagger} f_{\bar{\sigma}, L}$ & $\tilde{y}$ inv. + spin flip \\
\hline \hline & & & \\
\hline
\end{tabular}

winding of dislocations (cf. Fig. 6), but not of magnetic vortices. Consequently, it transforms $f_{\sigma}=b_{\sigma} V_{\sigma}$ onto a dual set of fermions $d_{\sigma} \equiv b_{\sigma}^{\dagger} V_{\sigma}$. The same dual fermions also arise under translation along $\hat{y}$, which takes $b_{\sigma} \rightarrow \sigma b_{\bar{\sigma}}^{\dagger}$ (cf. Table II).

We summarize the actions of all previously discussed microscopic symmetries on the fermionic partons in Table III. As in the case of bosonic partons, it is convenient to keep track of the $\mathrm{U}(1)$ spin-rotation symmetry by introducing the appropriate external probing field $\vec{A}$ [see Eq. (39)]. To lowest order in $\Delta$, it enters $\mathcal{L}_{f}$ and $\mathcal{L}_{f}^{\prime}$ by replacing $a_{\mu, \tilde{y}} \rightarrow a_{\mu, \tilde{y}}-$ $\frac{1}{4}(-1)^{\tilde{y}}\left[S A_{\mu}\right]_{\tilde{y}}$.

\section{B. Phases of fermionic partons and spins}

We now apply the formalism developed above to several specific phases of the fermionic-parton gauge theory. Following the same steps as for bosonic partons in Sec. IV B, we first study mean-field states without gauge-field dynamics. We then include gauge fluctuations and determine the quasiparticle content. Finally, we analyze the corresponding microscopic model.

\section{Trivial band insulator and intrawire $V B S$}

The fermionic partons are at unit filling and can form a trivial band insulator. To generate it, we perturb the parton gauge theory with the umklapp term of Eq. (77b). The resulting theory has the same form as the one describing Mott-gapped bosonic partons (see Sec. IV B 1), and its analysis is identical. In particular, we obtain the same microscopic model.

\section{Spin Hall insulator and easy-plane AFM}

Mean field. Consider a quantum spin Hall insulator of partons realized by the interwire couplings

$$
\delta \mathcal{L}_{\mathrm{QSH}}=g_{\mathrm{QSH}} \sum_{\sigma} f_{R, \tilde{y}+\sigma, \sigma}^{\dagger} f_{L, \tilde{y}-\sigma, \sigma}+\text { H.c., }
$$

where spin $\sigma=\uparrow(\downarrow)$ fermions reside on odd (even) dual wires. This model describes a fermionic band structure with gap $\propto g_{\mathrm{QSH}}$. The induced Lagrangian for $\vec{a}$, to lowest order in 
(a) Magnetic flux in QSH phase
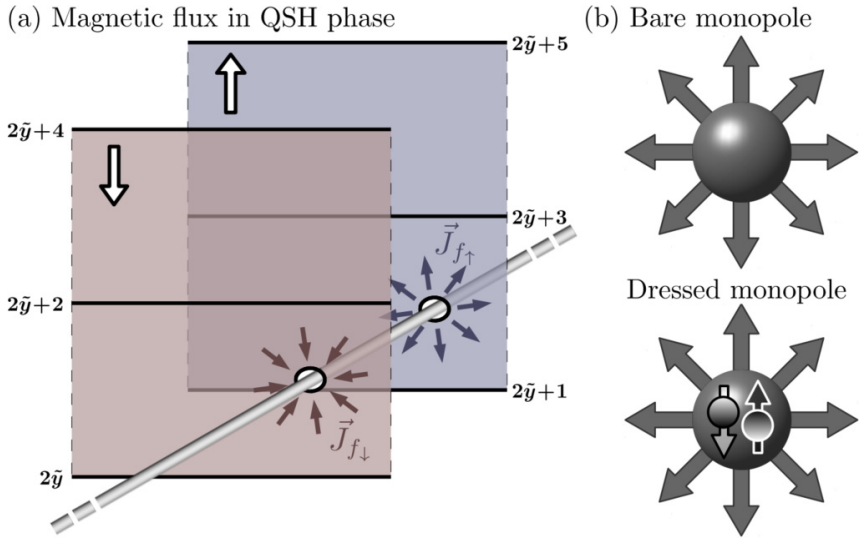

Dressed monopole

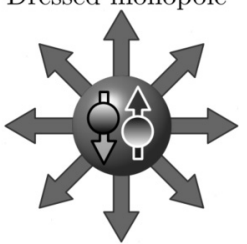

FIG. 11. (a) Adiabatically threading magnetic flux generates an electromotive force that creates an inward current of $\downarrow$ and an outward current of $\uparrow$ partons. When $2 \pi$ flux is introduced in this manner, a single $\downarrow$ particle and $\uparrow$ hole are pulled in. (b) Accordingly, fundamental monopoles become dressed by $f_{\downarrow}^{\dagger} f_{\uparrow}$ and carry spin 1. Their proliferation thus results in a phase with (spontaneously) broken U(1) spin-rotation symmetry, i.e., magnetic order.

$\Delta_{2}$, is

$$
\mathcal{L}_{\text {ind }}=\frac{i}{4 \pi} \epsilon_{\mu \nu}\left[S_{2} a_{\mu}^{s}\right]_{2 y}\left[\Delta_{2} a_{\nu}^{c}\right]_{2 y} \sim \frac{i}{2 \pi} \epsilon_{\mu \nu} a_{\mu}^{s} \partial_{y} a_{\nu}^{c},
$$

where $\left(S_{2}\right)_{y, y^{\prime}}=\delta_{y+2, y^{\prime}}+\delta_{y, y^{\prime}}$, and we have introduced the "charge" and "spin" gauge fields $\vec{a}^{c, s}$, which couple to $\rho_{2 y+1}^{c}=$ $\left(\rho_{2 \tilde{y}+1, \uparrow}+\rho_{2 \tilde{y}, \downarrow}\right)$ and $\rho_{2 y+1}^{s}=\left(\rho_{2 \tilde{y}+1, \uparrow}-\rho_{2 \tilde{y}, \downarrow}\right) / 2$, respectively.

This response reflects the well-known property of quantum spin Hall systems that a $2 \pi$ flux is accompanied by a spin 1 [118]. To see this explicitly, consider a configuration of $\vec{a}$ that includes a single $2 \pi$-flux tube penetrating through the plaquette delimited by $\tilde{y}_{0}$ and $\tilde{y}_{0}+1$ [see Fig. 11(a)]. In the $a_{2}=0$ gauge, such a configuration satisfies

$$
\oint_{\text {plaquette }} d \boldsymbol{l} \cdot \nabla \times \boldsymbol{a}=\int d x\left[\Delta a_{1}\right]_{y_{0}+1}=-2 \pi .
$$

Shifting $\varphi_{f, \tilde{y}} \rightarrow \varphi_{f, \tilde{y}}+\int^{x} d x^{\prime} a_{1, \tilde{y}}\left(x^{\prime}\right)$ transfers $a_{1}$ from the kinetic term in Eq. (81) to Eq. (83). In bosonized form, the latter becomes

$$
\delta \mathcal{L}_{\mathrm{QSH}}=\sum_{\sigma} \cos \left(2 \bar{\theta}_{\tilde{y}, \sigma}+\sigma \int^{x} d x^{\prime} \Delta_{2} a_{1, \tilde{y}}\right),
$$

with $\bar{\theta}_{\tilde{y}, \sigma}=\left(\phi_{\tilde{y}+\sigma, \sigma}^{R}-\phi_{\tilde{y}-\sigma, \sigma}^{L}\right) / 2$. During an adiabatic flux insertion, the argument of the cosine remains locked to its minimum and thus $\bar{\theta}_{\tilde{y}, \sigma} \rightarrow \bar{\theta}_{\tilde{y}, \sigma}-\sigma \int^{x} d x^{\prime} \Delta_{2} a_{1, \tilde{y}} / 2$. The resulting change in the parton numbers is $\delta Q_{\sigma}=\sigma$; recall $Q_{\sigma}$ are the same as for bosonic partons and given by Eq. (29). Inserting $2 \pi$ flux thus pulls in an $\uparrow$ parton and a $\downarrow$ hole, which together carry physical spin 1 .

Gauge fluctuations. Upon reinstating the status of $\vec{a}$ as a dynamical gauge field, we find its universal long-distance behavior to be unaffected by $\mathcal{L}_{\text {ind }}$. The field $\vec{a}^{s}$ describes modes near momentum $q=\pi$, which are massive according to the bare Maxwell term $\mathcal{L}_{\text {Maxwell }}$, and thus do not affect long-wavelength fluctuations. To lowest order in $\Delta$ and for frequencies and $x$ momenta small compared to the QSH gap, $\vec{a}$ is governed by

$$
\begin{aligned}
\mathcal{L}_{\text {MW }}= & \mathcal{L}_{\text {Maxwell }}+\frac{1}{2 \pi v_{F}}\left[\Delta a_{0}\right]_{\tilde{y}}^{2}+\frac{u_{F}}{2 \pi}\left[\Delta a_{1}\right]_{\tilde{y}}^{2} \\
& +\frac{\kappa d_{*}^{2}}{8 \pi \tilde{v}}\left(\partial_{x} a_{0, \tilde{y}}-\partial_{\tau} a_{1, \tilde{y}}\right)^{2} .
\end{aligned}
$$

Here, $\mathcal{L}_{\text {Maxwell }}$ is the bare gauge-field action of Eq. (81) and $d_{*}$ is a nonuniversal length scale proportional to the inverse QSH gap. While this effective action describes a propagating photon, the monopole operator $\mathcal{M}$ is strongly irrelevant. Its correlation function, according to Eq. (44), is

$$
C_{\boldsymbol{R}}^{\mathcal{M}}=\exp \left\{\frac{\kappa d_{*}}{8 \pi^{2} \tilde{v}} \int_{\boldsymbol{k}, \omega} \frac{\cos (\boldsymbol{k} \cdot \boldsymbol{R})-1}{k_{x}^{2}}\left[1-\frac{\kappa d_{*}^{2}}{4 \pi \tilde{v}}\left\langle\left|\varepsilon_{2}\right|^{2}\right\rangle_{0}\right]\right\},
$$

with $\varepsilon_{2}$ as in Sec. IV B 1 and $\boldsymbol{R}=\left(x, d_{*} y\right)$. The Gaussian average $\langle\ldots\rangle_{0}$, with respect to $\mathcal{L}_{\mathrm{MW}}$, is readily evaluated; we find the asymptotic behavior of $C_{\boldsymbol{R}}^{\mathcal{M}}$ is as given in Eq. (53) (see Appendix D 3 for details).

The reason for the rapid decay is that $\mathcal{M}$ attempts to introduce a gauge flux without the accompanying spin discussed above. Consider instead the "dressed" monopole $\mathcal{M}_{\text {dressed }} \sim f_{\downarrow}^{\dagger} f_{\uparrow} \mathcal{M}$ [see Fig. 11(b)]. Its correlation function reproduces Eq. (45), i.e., approaches a nonzero constant at long distances, and $\mathcal{M}_{\text {dressed }}$ spontaneously acquires an expectation value. Notice, however, that a term of the form $\delta \mathcal{L} \sim$ $\left(\mathcal{M}_{\text {dressed }}^{n}+\right.$ H.c. $)$ would explicitly break U(1) spin-rotation symmetry for any $n \neq 0$ and is therefore disallowed. The gauge field thus remains gapless in this phase, unlike in the trivial parton Mott insulator. For the details of these calculations, see Appendix D 3. In particular, the dressed monopole correlation function coincides with the one obtained by evaluating Eq. (10) using the singular configuration introduced in Sec. IV B 1.

Quasiparticles. The above analysis implies that, conversely, spin- $s$ operators must be accompanied by $2 \pi s$ gauge flux. Since $2 \pi$ is the fundamental monopole, there are no low-energy excitations with half-odd integer spin. Spinless excitations, such as $f_{\uparrow} f_{\downarrow}$, are charged under the emergent gauge field. They are thus subject to logarithmic confinement (cf. Sec. IV B 1).

Spin model. The parton QSH breaks the microscopic timereversal symmetry as well as translations by a single site in the $\hat{x}$ or $\hat{y}$ direction. It also exhibits spontaneous breaking of $\mathrm{U}(1)$ spin-rotation symmetry and an associated linear spectrum, as well as logarithmically confined neutral excitations. These properties exactly match those of an easy-plane AFM. Indeed, $\sum_{\tilde{y}} \delta \mathcal{L}_{\mathrm{QSH}}$ maps onto $\sum_{y} \mathcal{L}_{t}$, which generates the easy-plane AFM described in Sec. III B 1.

\section{Mixed insulators and interwire VBS}

Mean field. Consider now an integer quantum Hall state for one parton while the second forms a trivial band insulator. This is achieved, e.g., by introducing

$$
\delta \mathcal{L}_{\mathrm{MI}}=g_{t} f_{2 \tilde{y}+2, \downarrow, L}^{\dagger} f_{2 \tilde{y}, \downarrow, R}+g_{u} f_{2 \tilde{y}+1, \uparrow, R}^{\dagger} f_{2 \tilde{y}+1, \uparrow, L}+\text { H.c. }
$$


At the mean-field level, the fermionic partons constitute gapped spin- $\frac{1}{2}$ quasiparticles. The induced action for the gauge field $\vec{a}$ is

$$
\mathcal{L}_{\mathrm{CS} \text { odd }}=\frac{i}{4 \pi}\left[S_{2} a_{1}\right]_{2 \tilde{y}+1}\left[\Delta_{2} a_{0}\right]_{2 \tilde{y}+1},
$$

as expected for a quantum Hall state at unit filling.

Gauge fluctuations. When the gauge field $\vec{a}$ is promoted to a dynamical variable, it acquires a mass through the ChernSimons term. Monopoles are thus strongly irrelevant and can be safely discarded. The external probing field $\vec{A}$ can be included in Eq. (90) by replacing $a_{\mu, 2 \tilde{y}} \rightarrow a_{\mu, 2 \tilde{y}}+\frac{1}{4}\left[S A_{\mu}\right]_{2 \tilde{y}}$ (without modifying the bare Maxwell term). Integrating out $a_{\mu}$ does not result in a Chern-Simons term for $\vec{A}$, which raises the possibility that the microscopic time-reversal symmetry is preserved. Indeed, it translates into the combination of fermionic time-reversal and $y$-translation symmetries (cf. Table III), which is preserved by the parton band structure in Eq. (89).

Quasiparticles. The Chern-Simons term in Eq. (90) attaches $2 \pi$ emergent gauge flux to the fermionic mean-field excitations, converting them into bosonic quasiparticles. The way that $\vec{A}$ enters in Eq. (90) (see above) implies that the flux of $\vec{a}$ carries physical spin $\frac{1}{2}$. There are, thus, two types of bosonic quasiparticles, one with spin 1 and one with spin 0 .

Spin model. The $y$-translation symmetry is broken, while $\tilde{y}$ inversion is preserved (cf. Table II). Moreover, U(1) spin-rotation, time-reversal, and $x$-translation symmetries all remain intact. These symmetry properties, along with the integer-spin quasiparticles, identify the phase as an interwire VBS. Indeed, translating $\delta \mathcal{L}_{\mathrm{MI}}$ to the microscopic spin variables, we find the wire construction of Eq. (54), which realizes an interwire VBS.

\section{Generic Katrix and chiral spin liquid}

Mean field. Consider now quantum Hall states characterized by a nonsingular $2 \times 2 \mathrm{~K}$ matrix

$$
K=\left(\begin{array}{ll}
m_{\uparrow} & m_{0} \\
m_{0} & m_{\downarrow}
\end{array}\right),
$$

where $m_{\sigma}$ are odd integers and $\operatorname{det}[K] \neq 0$. The corresponding wire construction was worked out in Ref. [51] and is given by

$$
\delta \mathcal{L}_{K}=\tilde{g} \cos \left(\left[\Delta_{2} \varphi_{f, \sigma}\right]_{\tilde{y}}-m_{\sigma}\left[S_{2} \theta_{f, \sigma}\right]_{\tilde{y}}-2 m_{0} \theta_{f, \tilde{y}, \bar{\sigma}}\right) .
$$

For generic $m_{\sigma}, m_{0}$ such a state exhibits a quantum Hall effect (associated with the total charge), a spin quantum Hall effect (associated with the relative charge), and a quantum spin Hall effect that connects the total and relative charges. In terms of $q^{T}=(1,1)$ and $s^{T}=(1,-1) \mathrm{m}$, these are given by $v_{c c}=q^{T} K^{-1} q, v_{s s}=s^{T} K^{-1} s$, and $v_{c s}=q^{T} K^{-1} s$, respectively. Integrating out the matter field we find, at leading order in $\Delta_{2}$,

$$
\mathcal{L}_{\mathrm{CS} K}=\frac{i}{4 \pi} \sum_{i, j=c / s} v_{i j}\left[S_{2} a_{0}^{i}\right]_{2 y+2}\left[\Delta_{2} a_{1}^{j}\right]_{2 y+2},
$$

where we have introduced charge and spin gauge fields $\vec{a}_{2 y+1}^{c / s}=\left(\vec{a}_{2 \tilde{y}+1} \pm \vec{a}_{2 \tilde{y}}\right) / 2$. The quasiparticles, at the mean-field level, are anyons and carry fractional charges under $\vec{a}$. They can be determined by a standard $K$-matrix analysis (see, e.g., Ref. [47]).

Gauge fluctuations. Upon reinstating the dynamics of $\vec{a}$, governed by $\mathcal{L}_{\mathrm{MW}}+\mathcal{L}_{\mathrm{CS} K}$, we find two distinct cases. For $v_{c c}=0$, the gauge field remains gapless, and monopoles are important. The assumption of nonsingular $K$ implies a nonzero spin Hall response. Therefore, U(1) spin-rotation symmetry is spontaneously broken, as in the special case $m_{0}=0$ and $m_{\sigma}=\sigma$ (cf. Sec. V B 2). By contrast, for nonzero $v_{c c}$, the gauge field is massive, and monopoles can be safely discarded.

Recall that the microscopic time-reversal symmetry acts as a duality transformation on the partons. Specifically, it attaches $-2 \pi \sigma$ flux to the fermions (followed by particle-hole transformation, see Sec. V). Therefore, $K$-matrix states for the $f_{\sigma}$ fermions and for the dual $d_{\sigma}$ fermions are related by $-K_{f}=K_{d}-2 \sigma^{z}$. For non-zero $v_{c c}$, the two $K$ matrices cannot coincide, and time-reversal symmetry is broken explicitly. To obtain the physical response, we include the external probing field $\vec{A}$ in Eq. (93), according to $a_{\mu, \tilde{y}} \rightarrow a_{\mu, \tilde{y}}-$ $\frac{1}{4}(-1)^{\tilde{y}}\left[S A_{\mu}\right]_{\tilde{y}}$. Integrating out the emergent gauge field we obtain

$$
\mathcal{L}_{A \mathrm{CS}}=-\frac{i}{8 \pi n}\left[S A_{1}\right]_{\tilde{y}}\left[\Delta A_{0}\right]_{\tilde{y}} \sim-\frac{i}{8 \pi n} \epsilon_{\mu \nu} A_{\mu} \partial_{y} A_{\nu},
$$

where $n \equiv v_{c c} \operatorname{det}[K] / 2$. Consequently, the phase is chiral with topologically protected edge states.

Quasiparticles. To characterize the quasiparticles for $n \neq$ 0 , we adopt the strategy employed in Sec. IV B 4. To each fermion, we attach $m_{\sigma}$ fluxes of their own species and $m_{0}$ fluxes of the opposite one; on an operator level, we define $\eta_{\tilde{y}, \sigma}=\varphi_{f, \tilde{y}, \sigma}-\frac{m_{\sigma}}{2}\left[S_{2} \tilde{\varphi}_{f, \sigma}\right]_{\tilde{y}}-m_{0} \tilde{\varphi}_{f, \bar{\sigma}, \tilde{y}}$. The corresponding $\beta_{\sigma}=e^{-i \eta_{\sigma}}$ particles are bosons; they are governed by the schematic Lagrangian

$$
\mathcal{L}_{\beta}=i \sum_{\sigma} \vec{j}_{\sigma}^{\beta} \cdot\left(\vec{c}_{\sigma}+\frac{\sigma}{2} \vec{A}\right)-i \frac{\left(c_{\uparrow}-c_{\downarrow}\right) d\left(c_{\uparrow}-c_{\downarrow}\right)}{8 \pi n} .
$$

Notice that this Lagrangian is the same as Eq. (62). The quasiparticles thus carry spin $1 / 2 n$ and acquire statistical phases of $\pi / 2 n$ upon clockwise exchange. The case $n=0$ can be analyzed as in Sec. V B 2. In particular, these phases feature linearly dispersing Goldstone modes associated with the broken U(1) spin-rotation symmetry and logarithmically confined topological excitations.

Spin model. The response to the external probing field, Eq. (94), implies that the microscopic phase is a chiral QSL. Translating the interwire coupling in Eq. (92) to microscopic variables, we find

$$
\delta \mathcal{L}_{\mathrm{CSL}}^{\prime}=\tilde{g} \cos \left([\Delta \Phi]_{\tilde{y}}-2 m_{0}[S \Theta]_{\tilde{y}}+m_{\tilde{y}}\left[S_{2} S \Theta\right]_{\tilde{y}}\right),
$$

with $m_{2 y \pm 1 / 2}=m_{\uparrow / \downarrow} \mp 1$. For nonsingular $K \neq \sigma^{z}$, these interwire couplings explicitly break time-reversal symmetry. The arguments of all cosines in $\delta \mathcal{L}_{\mathrm{CSL}}^{\prime}$ commute and can flow to strong coupling simultaneously. They are, moreover, linearly independent and can thus generate a gapped phase for nonzero $n$, i.e., $m_{\tilde{y}}+m_{\tilde{y}+1} \neq 2 m_{0}$. To identify its quasiparticles and edge structure, we introduce chiral modes

$$
\tilde{\phi}_{\chi, y}=\Phi_{y}+\chi 2 n \Theta_{y}+2 m_{y+1} \Theta_{y+1}-2 m_{y} \Theta_{y-1},
$$


which satisfy

$$
\left[\partial_{x} \tilde{\phi}_{\chi, y}, \tilde{\phi}_{\chi^{\prime}, y^{\prime}}\right]=i \chi 4 \pi n \delta_{y, y^{\prime}} \delta_{\chi, \chi^{\prime}} \delta\left(x-x^{\prime}\right) .
$$

Crucially, this change of variables preserves the locality of both the intrawire and interwire terms; the latter take the form $\delta \mathcal{L}_{\mathrm{CSL}}^{\prime}=\cos \left(\tilde{\phi}_{R, y+1}-\tilde{\phi}_{L, y}\right)$. Domain walls in these cosines carry spin $1 / 2 n$ and acquire exchange phases of $\pi / 2 n$.

Finally, for $n=0$ the system is gapless, which can be seen by summing the arguments of all cosines in Eq. (96), i.e., $\sum_{y}\left[\tilde{\phi}_{R, y+1}-\tilde{\phi}_{L, y}\right]=4 n \sum_{y} \Theta_{y}$. This particular linear combination thus remains unpinned for $n=0$. Its conjugate describes the Goldstone mode associated with the spontaneously broken U(1) spin-rotation symmetry, precisely as in Sec. III B 1.

\section{BCS superconductor and $\mathbb{Z}_{2}$ spin liquid}

Mean field. As a final example, consider now a BCS superconductor of fermionic partons. To generate pairing, we introduce interwire hopping for the Cooper-pair operator $\Psi_{f \text {-pair }, 2 y+1} \equiv f_{2 \tilde{y}+1 \uparrow, R} f_{2 \tilde{y}, \downarrow, L}$, i.e.

$$
\delta \mathcal{L}_{f \text {-pair }}=g_{f \text {-pair }} \Psi_{f \text {-pair }, 2 y+1}^{\dagger} \Psi_{f \text {-pair }, 2 y-1}+\text { H.c. }
$$

When $g_{f \text {-pair }}$ flows to strong coupling, $\Psi_{f \text {-pair }}$ spontaneously acquires an expectation value (cf. Sec. IIIB 1). Vortices in the phase of this condensate, created by $m_{2 y}=$ $e^{-i\left(\tilde{\varphi}_{f, \uparrow, 2 \tilde{y}}+\tilde{\varphi}_{f, \downarrow, 2 \tilde{y}-1}\right) / 2}$, are logarithmically confined. While $\delta \mathcal{L}_{f \text {-pair }}$ renders the umklapp term in Eq. (77b) irrelevant, the backscattering term

$$
\delta \mathcal{L}_{\mathrm{bs}}=g_{\mathrm{bs}} f_{2 \tilde{y}+1, \uparrow, R}^{\dagger} f_{2 \tilde{y}+1, \uparrow, L} f_{2 \tilde{y}, \downarrow, L}^{\dagger} f_{2 \tilde{y}, \downarrow, R}+\text { H.c. }
$$

can flow to strong coupling. When it does, a fully gapped phase with fermionic spin- $\frac{1}{2}$ quasiparticles $f_{\sigma}$ obtains.

Gauge fluctuations. Since $\Psi_{f \text {-pair }}$ carries emergent gauge charge, its condensation leads to a Higgs mass. Monopoles are, therefore, strongly suppressed and can be safely discarded. Importantly, $\Psi_{f \text {-pair }}$ is neutral under the external probing field $\vec{A}$, so the microscopic $\mathrm{U}(1)$ spin-rotation symmetry is preserved. Indeed, the response to $\vec{A}$ is, at leading order in derivatives, described by a Maxwell action.

Quasiparticles. Gauge-field fluctuations that are rendered massive by a Higgs term do not affect the status of $f_{\sigma}$ as fermionic quasiparticles. They do, however, promote vortices $m$ to deconfined bosonic spin-0 quasiparticles. Being superconducting vortices, they are experienced as $\pi$ flux by the fermions, i.e., the two are mutual semions. Consequently, the two can combine into $\varepsilon=f_{\chi, \sigma} m^{\dagger}$, a spin- $\frac{1}{2}$ quasiparticle with bosonic self-statistics.

Spin model. The quasiparticle content characterizes a $\mathbb{Z}_{2}$ spin liquid that is, moreover, nonchiral and spin-rotation symmetric. Translating $\delta \mathcal{L}_{f \text {-pair }}$ and $\delta \mathcal{L}_{\text {bs }}$ to microscopic spin variables, we find

$$
\begin{aligned}
\delta \mathcal{L}_{\mathbb{Z}_{2}}^{\prime}= & g_{\text {bs }} \cos \left(2\left[S^{T} S \Theta\right]_{2 y-1}\right) \\
& +g_{f \text {-pair }} \cos \left(\left[\Delta^{T} \Delta \Phi\right]_{2 y}+2\left[S^{T} S \Theta\right]_{2 y-1}\right) .
\end{aligned}
$$

The arguments of these cosines are linear combinations of those in Eq. (69). Consequently, they lead to the same $\mathbb{Z}_{2}$ spin-liquid phase (see full analysis in Sec. IV B 5).

\section{SUMMARY AND DISCUSSION}

We have introduced exact, nonlocal mappings between arrays of spin- $\frac{1}{2}$ chains and parton gauge theories. Any parton model that separately conserves both species maps onto a local spin Hamiltonian. The challenge of deriving spin models that realize exotic ground states is thereby reduced to constructing parent Hamiltonians for simple phases of bosons or fermions. Conversely, any $S^{z}$-conserving coupling between spins transforms into a distinct interaction or hopping term for partons. The latter are obtained without reference to a specific mean-field Ansatz. They, therefore, retain not only information about the symmetries of the underlying spin model, but also about more subtle aspects, such as geometric frustration. In its presence, some symmetry-allowed terms in the dual parton description are absent. Geometric frustration may thus take the form of an emergent symmetry and, thereby, stabilize phases that would not readily form in more generic situations.

To demonstrate the versatility of this method, we showed how to recover trivial states and access topologically ordered ones. Relatively simple phases of partons already correspond to fractionalized ground states. As a first example, we derived parent Hamiltonians for Abelian chiral spin liquids. Here, knowing wire models of bosonic integer quantum Hall states was sufficient to immediately generate a parent Hamiltonian of the Kalmeyer-Laughlin chiral spin liquid, previously constructing by different means in Refs. [82,83]. Similarly, we obtained the wire model of a time-reversal-invariant $\mathbb{Z}_{2}$ spin liquid as a simple $s$-wave BCS superconductor of fermionic partons. If they instead form a topological superconductor, such as $p_{x} \pm i p_{y}$, the resulting QSL will be non-Abelian.

When the partons themselves form nontrivial phases, an even wider range of exotic microscopic ground states is realized. The framework introduced here applies to such cases with no additional difficulties, once a (coupled-wire) parent Hamiltonian of the parton phase is known. We have illustrated this capability by the example of a general $2 \times 2 \mathrm{~K}$-matrix state of fermionic partons. Based on the known fermionic wire constructions, we easily generated wire models for a range of chiral spin liquid that were not previously available. Explicit parent Hamiltonians for even more exotic states, such as the non-Abelian Read-Rezayi sequence of fractional quantum Hall states, are also known and can likewise be used to generate concrete spin models.

We primarily focused on fully gapped states. However, the dual description of spin-chain arrays in terms of fermions may be able to capture exotic gapless phases and unconventional quantum phase transitions as well. One example of the latter arises at the transition between the easy-plane AFM and the intrawire VBS. It maps onto a coupled-wire model of compact $\mathrm{QED}_{3}$ with two boson or fermion species. This is precisely the effective field theory that was derived using different methods in Ref. [109]. Its fate in the infrared is thought to be confining (and consequently the transition to be first order). A stable gapless theory may instead arise in various ways: (i) at a transition between different phases; (ii) in the presence of emergent symmetries of the parton field theory that may arise due to geometric frustration; (iii) when the emergent fermions are doped to form a Fermi surface that suppresses monopole events. All three scenarios feature nontrivial gauge- 
field dynamics, which places them beyond the capability of conventional wire constructions. They should, however, be amenable to exploration within the formalism developed here.

Finally, we mention two possible generalizations of the methods developed here. The first is to itinerant electron systems. There, decomposing microscopic electron operators as $c_{\sigma}=b f_{\sigma}$ allows exploration of many exotic ground states. Extending our approach to wire arrays with both spin and charge modes may allow well-controlled access to those phases, and provide concrete model systems where they arise. A second interesting direction is given by spin models that do not conserve $S^{z}$, such as the celebrated Kitaev honeycomb model [11]. Systems without U(1) symmetries are not readily describable within Abelian bosonization. Instead, coupled- wire techniques based on non-Abelian bosonization have been used successfully in similar contexts [71,85]. Generalizing our methods to these systems could provide a much-desired bridge between fine-tuned solvable models and mean-field studies of generic ones.

\section{ACKNOWLEDGMENTS}

We thank J. Alicea, O. Motrunich, and C. Wang for many illuminating discussions. This work was supported by the Israel Science Foundation and by the Minerva foundation with funding from the Federal German Ministry for Education and Research. Part of this research was performed at the Aspen Center for Physics, which is supported by National Science Foundation Grant No. PHY-1607611.

\section{APPENDIX A: ENERGY COST OF TOPOLOGICAL DEFECTS}

\section{Energy cost of domain walls in the VBS phase of a one-dimensional spin chain}

To describe the VBS phase, consider the bosonized action of a U(1) spin chain given by

$$
\mathcal{S}_{\mathrm{VBS}}=\int_{x, \tau}\left[\frac{1}{2 \pi v K}\left(\partial_{\tau} \Theta\right)^{2}+\frac{v}{2 \pi K}\left(\partial_{x} \Theta\right)^{2}+\frac{v \tilde{g}_{4 \pi}}{16 \pi K d_{0}^{2}} \cos (4 \Theta)\right],
$$

with $K$ such that the dimensionless coupling constant $\tilde{g}_{4 \pi}<0$ flows to strong coupling. The minima of the cosine potential, $\Theta_{\min }=\pi n / 2$, correspond to different topological sectors, labeled by the integer $n$. On length scales larger than $d_{*}$, where $\tilde{g}_{4 \pi}$ has become of order unity, it is appropriate to expand the cosine in a single topological sector, i.e., replace $\frac{v \tilde{g}_{4 \pi}}{16 \pi K d_{0}^{2}} \cos (4 \Theta) \rightarrow$ $\frac{v}{2 \pi K d_{*}^{2}}(\Theta-\pi n / 2)^{2}$. To find the energy cost of domain walls, we allow the system to transition between different topological sectors as a function of space, i.e., $n \rightarrow n_{x}$ and

$$
\mathcal{S}_{\text {eff }}[n]=\frac{1}{2 \pi v K} \int_{x, \tau}\left[\left(\partial_{\tau} \Theta\right)^{2}+v^{2}\left(\partial_{x} \Theta\right)^{2}+\frac{v^{2}}{d_{*}^{2}} \Theta^{2}-\frac{\pi v^{2}}{d_{*}^{2}} \Theta n_{x}+\frac{\pi^{2} v^{2}}{4 d_{*}^{2}} n_{x}^{2}\right] .
$$

The energy cost of forcing the system into different topological sectors, relative to the uniform $n=0$ vacuum, is given by

$$
\begin{aligned}
\Delta E[n] & =-\lim _{T \rightarrow 0} T \log \left(\frac{\mathcal{Z}[n]}{\mathcal{Z}[0]}\right)=-\lim _{T \rightarrow 0} T \log \left\langle e^{\mathcal{S}[0]-\mathcal{S}[n]}\right\rangle_{0} \\
& =\frac{\pi v}{8 K d_{*}^{2}} \int_{x} n_{x}^{2}-\left.\frac{v^{2}}{8 K^{2} d_{*}^{4}} \int_{x, x^{\prime}} n_{x} n_{x^{\prime}}\left\langle\Theta_{x} \Theta_{x^{\prime}}\right\rangle_{0}\right|_{\omega=0}=\left.\frac{v^{2}}{8 K^{2} d_{*}^{2}} \int_{x, x^{\prime}} n_{x} n_{x^{\prime}}\left\langle\partial_{x} \Theta_{x} \partial_{x^{\prime}} \Theta_{x^{\prime}}\right\rangle_{0}\right|_{\omega=0},
\end{aligned}
$$

where the last equality uses the specific form of the correlation function $\left\langle\Theta_{x} \Theta_{x^{\prime}}\right\rangle$ according to Eq. (A2). For a generic configuration of domain walls parametrized by $n(x)=\sum_{i} \alpha_{i} H\left(x-x_{i}\right)$ with $\alpha_{i} \in \mathbb{Z}$, the energy is given by

$$
\Delta E[\{\alpha\}]=\left.\frac{v^{2}}{8 K^{2} d_{*}^{2}} \sum_{i, j} \alpha_{i} \alpha_{j}\left\langle\Theta_{x_{i}} \Theta_{x_{j}}\right\rangle_{0}\right|_{\omega=0}=\frac{\pi v}{16 K d_{*}} \sum_{i, j} \alpha_{i} \alpha_{j} e^{-\left|x_{i}-x_{j}\right| / d_{*}} .
$$

In particular, the energy cost of a single domain wall is given by the prefactor $\Delta E_{\mathrm{DW}}=\frac{\pi v}{16 K d_{*}}$.

The trial function provided in the main text, $\Theta_{\mathrm{DW}}(x)=\tan ^{-1}\left[e^{\left(x-x_{0}\right) / \xi}\right]$, produces a variational energy cost of $\Delta E_{\mathrm{DW}}[\xi]=$ $\frac{v}{4 \pi K}\left(1 / \xi+\xi / d_{*}^{2}\right)$ for the renormalized action, i.e., Eq. (A1), with $\tilde{g}_{4 \pi} \rightarrow-d_{0}^{2} / d_{*}^{2}$. Its minimal value, attained for $\xi=d_{*}$, is given by $\Delta E_{\mathrm{DW}}=\frac{v}{2 \pi K d_{*}}$ and is parametrically the same as the result of Eq. (A4). The somewhat smaller numerical value relative to the previous calculation arises because there, the cosine was replaced by a parabolic potential centered around the nearest minimum.

\section{Energy cost of magnetic vortices in the easy-plane AFM}

We follow the same strategy as for the one-dimensional VBS domain walls. Expanding the cosine of Eq. (18a) in topological sectors, denoted by $n_{x, y+1 / 2}$, we obtain

$$
\mathcal{S}_{\mathrm{eff}}[n]=\frac{K}{2 \pi v} \int_{x, \tau} \sum_{y}\left[\left(\partial_{\tau} \Phi_{y}\right)^{2}+v^{2}\left(\partial_{x} \Phi_{y}\right)^{2}+\frac{v^{2}}{d_{*}^{2}}\left([\Delta \Phi]_{y+\frac{1}{2}}-2 \pi n_{x, y+\frac{1}{2}}\right)^{2}\right],
$$


where $d_{*}$ is the length scale at which $\tilde{g}_{t}=2 \pi d_{0}^{2} g_{t} / v K$ reaches order unity. In this case, $n$ can be interpreted as counting the magnetic flux tubes in an external probing field. Consider a magnetic field $B(x)$ in the gauge $A_{1}=0$, i.e., $A_{2}=\int_{x} B(x)$. Incorporating the probing field via minimal coupling amounts to replacing $\Delta \Phi \rightarrow \Delta \Phi-A_{2}$ above, which identifies $n=$ $\frac{1}{2 \pi} \int_{x} B(x)$. The energy cost for a given configuration $n$ can be computed as in the one-dimensional case, i.e.,

$$
\Delta E[n]=\left.2 v^{2} K^{2} d_{*}^{-2} \int_{x, x^{\prime}} \sum_{y, y^{\prime}} n_{x, y+\frac{1}{2}} n_{x^{\prime}, y^{\prime}+\frac{1}{2}}\left\langle\partial_{x} \Phi_{x, y} \partial_{x^{\prime}} \Phi_{x^{\prime}, y^{\prime}}\right\rangle_{0}\right|_{\omega=0} .
$$

Parametrizing $n$ in terms of strength $\alpha_{i}$ vortices at positions $\left(x_{i}, \tilde{y}_{i}\right)$, i.e., $n_{x, y+\frac{1}{2}}=\sum_{i} \alpha_{i} \delta_{y, y_{i}} H\left(x-x_{i}\right)$, we arrive at the final expression

$$
\Delta E[\{\alpha\}]=\left.2 v^{2} K^{2} d_{*}^{-2}\left\langle\left(\sum_{i} \alpha_{i} \Phi_{x_{i}, y_{i}}\right)^{2}\right\rangle_{0}\right|_{\omega=0}=\frac{v K}{2 \pi d_{*}} \sum_{i, j} \alpha_{i} \alpha_{j} \int_{k_{x}, k_{y}} \frac{\cos \left[\boldsymbol{k} \cdot\left(\boldsymbol{R}_{i}-\boldsymbol{R}_{j}\right)\right]}{k_{x}^{2}+\left|\Delta_{k_{y}}\right|^{2}},
$$

with $\boldsymbol{R}_{i}=\left(x_{i}, d_{*} \tilde{y}_{i}\right)$ and $\boldsymbol{k}=\left(k_{x}, k_{y}\right)$. The $y$ momentum $k_{y}$ is measured in units of $d_{*}^{-1}$ and $\Delta_{k_{y}} \equiv\left(e^{i d_{*} k_{y}}-1\right) / d_{*}$. If the total number of vortices $N_{\mathrm{v}} \equiv \sum_{i} \alpha_{i} \neq 0$, the energy diverges logarithmically with the size of the system. When $N_{\mathrm{v}}=0$, the energy cost is finite; for a single vortex-antivortex pair we find

$$
\lim _{\left|\boldsymbol{R}_{1}-\boldsymbol{R}_{2}\right| \rightarrow \infty} \Delta E_{\boldsymbol{R}_{1}, \boldsymbol{R}_{2}}=\frac{2 v K}{d_{*}} \log \left(\frac{\left|\boldsymbol{R}_{1}-\boldsymbol{R}_{2}\right|}{2 d_{*}}\right) .
$$

Importantly, this calculation does not make any reference to a specific form of the vortex. Instead, it fixes the topological properties and lets the functional integral over $\Phi$ find the optimal configuration. The same result can be obtained by considering a "trial" configuration of the form

$$
\Phi_{\text {trial }}=\arg \left[\left(x-x_{1}\right) / \xi+i\left(y-\tilde{y}_{1}\right)\right]-\arg \left[\left(x-x_{2}\right) / \xi+i\left(y-\tilde{y}_{2}\right)\right],
$$

where $\xi$ is a variational parameter. Computing the corresponding energy at large vortex-antivortex separation, one finds

$$
E_{\text {trial }}[\xi]=\frac{K}{2 \pi v} \int_{x} \sum_{y}\left[v^{2}\left(\partial_{x} \Phi_{\text {trial }}\right)^{2}+\frac{v^{2}}{d_{*}^{2}}\left[\Delta \Phi_{\text {trial }}\right]^{2}\right]=\frac{2 v K}{d_{*}} \frac{d_{*}^{2}+\xi^{2}}{2 \xi d_{*}} \log \left(\frac{\left|\boldsymbol{R}_{i}-\boldsymbol{R}_{j}\right|}{2 \xi}\right)+O(1)
$$

with $\boldsymbol{R}_{i}=\left(x_{i}, \xi y_{i}\right)$. To optimize $\xi$ within logarithmic accuracy, it is sufficient to focus on the prefactor of the logarithm; it is minimized for $\xi=d_{*}$ where Eq. (A10) reduces to the result provided in Eq. (A8).

\section{Energy cost of dislocations and bosonic partons in the intrawire VBS}

To generate the two-dimensional VBS with dimers along the wire direction $\hat{x}$, we introduce two different cosines per wire pair. We introduce dimensionless coupling constants $\tilde{g}_{u}>0$ and $\tilde{g}_{4 \pi}<0$, and write

$$
\delta \mathcal{L}_{\text {intra VBS }}=-\frac{v \tilde{g}_{u}}{4 \pi K d_{0}^{2}} \cos \left(2[\Delta \Theta]_{y+1 / 2}\right)+\frac{v \tilde{g}_{4 \pi}}{16 \pi K d_{0}^{2}} \cos \left(4 \Theta_{y}\right),
$$

where, for convenience, we have redefined $\Theta_{y} \rightarrow(-1)^{y}\left(\Theta_{y}+\pi y / 2\right)$. Beyond the length scales $d_{*}$ and $l_{*}$, where the coupling constants renormalize to order unity, we expand the action in topological sectors. Labeling said sectors by integers $n$ and $p$, we obtain

$$
\mathcal{S}_{\text {eff }}[n, p]=\frac{1}{2 \pi v K} \int_{x, \tau} \sum_{y}\left[\left(\partial_{\tau} \Theta_{y}\right)^{2}+v^{2}\left(\partial_{x} \Theta_{y}\right)^{2}+\frac{v^{2}}{d_{*}^{2}}\left([\Delta \Theta]_{y+\frac{1}{2}}-\pi n_{x, y+\frac{1}{2}}\right)^{2}+\frac{v^{2}}{l_{*}^{2}}\left(\Theta_{y}-\frac{\pi}{2} p_{x, y}\right)^{2}\right] .
$$

As before, we parametrize the integer functions $n$ and $p$ by the locations and strengths of topological defects, i.e., $n=$ $\sum_{i} \alpha_{i} H\left(x-x_{i}\right) \delta_{\tilde{y}, \tilde{y}_{i}}$ and $p=\sum_{i} \beta_{i} H\left(x-X_{i}\right) \delta_{y, Y_{i}}$. The energy cost for a general configuration naturally decomposes into a manifestly local, system-size-independent contribution, and a nonlocal one that may be IR divergent, i.e., $\Delta E[n, p]=$ $\Delta E_{\text {local }}[n, p]+\Delta E_{\text {nonlocal }}\left[n-\frac{1}{2} \Delta p\right]$ with

$$
\begin{aligned}
\Delta E_{\text {local }} & =\left.\frac{v^{2}}{2 K^{2} d_{*}^{2}} \sum_{i, j} \alpha_{i} \alpha_{j}\left\langle\Theta_{x_{i}, y_{i}} \Theta_{x_{j}, y_{j}}\right\rangle_{0}\right|_{\omega=0}+\left.\frac{v^{2}}{8 K^{2} l_{*}^{2}} \sum_{i, j} \beta_{i} \beta_{j}\left\langle\Theta_{x_{i}, y_{i}} \Theta_{x_{j}, y_{j}}\right\rangle_{0}\right|_{\omega=0}, \\
\Delta E_{\text {nonlocal }} & =\left.\frac{v^{2}}{2 K^{2} d_{*}^{2} l_{*}^{2}} \int_{x, x^{\prime}} \sum_{y, y^{\prime}}\left(n_{x, y+\frac{1}{2}}-\frac{1}{2}[\Delta p]_{x, y+\frac{1}{2}}\right)\left(n_{x^{\prime}, y^{\prime}+\frac{1}{2}}-\frac{1}{2}[\Delta p]_{x^{\prime}, y^{\prime}+\frac{1}{2}}\right)\left\langle\Theta_{x, y} \Theta_{x^{\prime}, y^{\prime}}\right\rangle_{0}\right|_{\omega=0} .
\end{aligned}
$$

A priori $n$ and $p$ are independent, and the lowest energy cost for a given $n$ must be found by optimizing over all possible $p$. Fortunately, this minimization can be avoided when the density of defects $n$ is low; there, the optimal $p$ can be inferred based on general considerations. 
Consider a single dislocation-antidislocation pair defined by $\alpha_{1}=1$ and $\alpha_{2}=-1$, i.e., $n_{x, \tilde{y}}=H\left(x-x_{1}\right) \delta_{\tilde{y}, \tilde{y}_{1}}-$ $H\left(x-x_{2}\right) \delta_{\tilde{y}, \tilde{y}_{2}}$. The corresponding $p$ can be deduced as follows: (i) To avoid energies that diverge with system size, the topological sectors must match asymptotically, i.e., $\lim _{x \rightarrow \infty}\left(n_{x, \tilde{y}}-[\Delta p]_{x, \tilde{y}} / 2\right)=0$. (ii) The minimal number of $p$ defects within this constraint has exactly one of strength $\beta_{i}=2$ for each wire $Y_{i} \in\left[\tilde{y}_{1}, \tilde{y}_{2}\right]$. (iii) Their optimal locations are along the line connecting $\tilde{\boldsymbol{r}}_{1}=\left(x_{1}, \tilde{y}_{1}\right)$ and $\tilde{\boldsymbol{r}}_{2}=\left(x_{2}, \tilde{y}_{2}\right)$. To determine the energy for distances much larger than $l_{*}$, we approximate

$$
\left.\left\langle\Theta_{x, y} \Theta_{x^{\prime}, y^{\prime}}\right\rangle_{0}\right|_{\omega=0}=\frac{K d_{*}}{4 \pi v} \int_{k_{x}, k_{y}} \frac{\cos \left[\boldsymbol{k} \cdot\left(\boldsymbol{R}-\boldsymbol{R}^{\prime}\right)\right]}{k_{x}^{2}+\left|\Delta_{k_{y}}\right|^{2}+l_{*}^{-2}} \approx \frac{\pi K l_{*}^{2}}{v} \delta\left(x-x^{\prime}\right) \delta_{y, y^{\prime}},
$$

where $\boldsymbol{R}=\left(x, d_{*} y\right)$. [When appearing in discrete sums as in $\Delta E, \delta\left(x_{i}-x_{j}\right)$ is to be understood as $d_{0}^{-1} \delta_{x_{i}, x_{j}}$, where the microscopic length scale $d_{0}$ acts as a UV cutoff.] In this limit we find simplified expressions for the local and nonlocal contributions to the energy, which in case of a single dislocation-antidislocation pair give

$$
\begin{aligned}
\Delta E_{\text {local }} & =\frac{\pi v l_{*}^{2}}{2 K d_{0} d_{*}^{2}} \sum_{i} \alpha_{i}^{2}+\frac{\pi v}{8 K d_{0}} \sum_{i} \beta_{i}^{2}=\frac{\pi v l_{*}^{2}}{K d_{0} d_{*}^{2}}+\frac{\pi v}{2 K d_{0}}\left(\tilde{y}_{2}-\tilde{y}_{1}\right), \\
\Delta E_{\text {nonlocal }} & =\frac{\pi v}{2 K d_{*}^{2}} \int_{x} \sum_{y}\left(n_{x, y+\frac{1}{2}}-\frac{1}{2}[\Delta p]_{x, y+\frac{1}{2}}\right)^{2}=\frac{\pi v}{2 K d_{*}^{2}} \frac{\left(x_{2}-x_{1}\right)}{1+\left(\tilde{y}_{2}-\tilde{y}_{1}\right)^{-1}} .
\end{aligned}
$$

Topological defects in the intrawire VBS phase are thus linearly confined. As for the case of magnetic vortices, the same conclusion can be reached by studying appropriate trial states. For the Ising-AFM phase, the sign of $\tilde{g}_{4 \pi}$ in Eq. (A11) is reversed, but the analysis is otherwise identical, i.e., defects are also linearly confined.

\section{Energy cost of bosonic-parton vortices in the correlated Mott insulator}

In the correlated Mott insulator (cf. Sec. IV B 3), there are two cosines per wire pair, i.e.,

$$
\delta \mathcal{L}_{\downarrow}+\delta \mathcal{L}_{\uparrow}=\frac{v_{B} \tilde{g}_{u}}{\pi d_{0}^{2}} \cos \left(\left[\Delta_{2} \varphi_{\downarrow}\right]_{2 \tilde{y}+1}\right)+\frac{u_{B} \tilde{g}_{t}}{\pi d_{0}^{2}} \cos \left(2 \theta_{2 \tilde{y}+1, \uparrow}\right) .
$$

Beyond the length scales $d_{*}$ and $l_{*}$, where coupling constants renormalize to order unity, we expand the action in the topological sectors. Labeling said sectors by the integer functions $n$ and $p$, we write

$$
\mathcal{S}_{\text {eff }}[n, p]=\mathcal{S}_{0}+\int_{x, \tau} \sum_{\tilde{y}}\left[\frac{2 v_{B}}{d_{*}^{2}}\left(\pi n_{x, 2 \tilde{y}+1}^{2}-\left[\Delta_{2} \varphi_{\downarrow}\right]_{2 \tilde{y}+1} n_{x, 2 \tilde{y}+1}\right)+\frac{2 u_{B}}{l_{*}^{2}}\left(\pi p_{x, 2 \tilde{y}+1}^{2}-2 \theta_{2 \tilde{y}+1, \uparrow} p_{x, 2 \tilde{y}+1}\right)\right],
$$

where $\mathcal{S}_{0}$ is the effective action of the trivial topological sector with $n=p=0$. Vortex-antivortex pairs are encoded in $n$, so we set $p$ to zero. To evaluate the energy cost for a given $n$, we need the Green function of $\Delta \varphi_{\downarrow}$ within the Gaussian theory $\mathcal{S}_{0}\left[\varphi_{\sigma}, \theta_{\sigma}, a\right]$. Its leading order in $k_{x}$ and $\Delta_{k_{y}}$ is

$$
\left.\left\langle\left|\Delta_{2 k_{y}} \varphi_{\downarrow}\right|^{2}\right\rangle\right|_{\omega=0}=\frac{\pi \alpha^{2}}{v_{B} d_{*}^{2}} \frac{1+\alpha^{2} d_{*}^{2}\left|\Delta_{k_{y}}\right|^{2}}{k_{x}^{2}+\left|\Delta_{k_{y}}\right|^{2}+\frac{\alpha^{2}}{d_{*}^{2}}},
$$

where the dimensionless parameter $\alpha^{2}=\frac{4 v_{B}}{\kappa \tilde{v}}$ encodes the ratio of boson and gauge-field velocities. For a single vortex-antivortex pair at positions $\boldsymbol{R}_{i}=\left(x_{i}, d_{*} \tilde{y}_{i}\right)$, with $\tilde{y}_{i}$ odd and $\left|\boldsymbol{R}_{1}-\boldsymbol{R}_{2}\right| \gg d_{*}$ we find

$$
\Delta E_{\boldsymbol{R}_{1}, \boldsymbol{R}_{2}}=\frac{v_{B}}{2 \pi d_{*}} \int_{k_{x}, k_{y}} \frac{2-2 \cos \left[\boldsymbol{k} \cdot\left(\boldsymbol{R}_{1}-\boldsymbol{R}_{2}\right)\right]}{k_{x}^{2}+\left|\Delta_{k_{y}}\right|^{2}+\frac{\alpha^{2}}{d_{*}^{2}}}=\frac{2 v_{B}}{d_{*}} f\left(\alpha^{2}\right)-\frac{2 v_{B}}{d_{*}} \sqrt{\frac{\pi d_{*}}{2 \alpha\left|\boldsymbol{R}_{1}-\boldsymbol{R}_{2}\right|}} e^{-\alpha\left|\boldsymbol{R}_{1}-\boldsymbol{R}_{2}\right| / d_{*}} .
$$

Here, the dimensionless integral $f(x)=\frac{1}{2} \int_{-\pi}^{\pi} d \theta \frac{1}{\sqrt{x+2-2 \cos (\theta)}}$ diverges logarithmically for $x \rightarrow 0$ but is otherwise finite. The energy required for creating an isolated vortex is thus $v_{B} / d_{*}$ times a number of order unity.

\section{APPENDIX B: DERIVATION OF THE BOSONIC-PARTON FIELD THEORY}

The equivalence between the microscopic coupled-wire model described in Sec. III B and the gauge theory for bosonic partons introduced in Sec. IV A can be shown using the methods of Refs. [95,96]. We begin with the gauge theory $\mathcal{S}=$ $\int_{x, \tau} \sum_{y}\left[\mathcal{L}_{b}+\mathcal{L}_{\text {Maxwell }}+\mathcal{L}_{b}^{\prime}\right]$ in the $a_{2}=0$ gauge, where

$$
\begin{aligned}
\mathcal{L}_{b} & =\frac{i}{\pi} \partial_{x} \theta_{\tilde{y}}\left(\partial_{\tau} \varphi_{\tilde{y}}-a_{0, \tilde{y}}\right)+\frac{v_{B}}{2 \pi}\left(\partial_{x} \varphi_{\tilde{y}}-a_{1, \tilde{y}}\right)^{2}+\frac{u_{B}}{2 \pi}\left(\partial_{x} \theta_{\tilde{y}}\right)^{2}, \\
\mathcal{L}_{\text {Maxwell }} & =\frac{\kappa}{4 \pi \tilde{v}}\left[\Delta a_{0}\right]_{y}^{2}+\frac{\kappa \tilde{v}}{4 \pi}\left[\Delta a_{1}\right]_{y}^{2} .
\end{aligned}
$$


We here omit the redundant label $\sigma=(-1)^{y+1}$ to lighten the notation. The final term $\mathcal{L}_{b}^{\prime}$ contains exponentially decaying interwire interactions. Specifically, density-density and current-current interactions

$$
\mathcal{L}_{b}^{\prime}=\sum_{y^{\prime}}\left\{\left[\Delta \partial_{x} \theta\right]_{y}\left(W_{1}\right)_{y, y^{\prime}}\left[\Delta \partial_{x} \theta\right]_{y^{\prime}}+\left[\Delta \partial_{x} \varphi\right]_{y}\left(W_{2}\right)_{y, y^{\prime}}\left[\Delta \partial_{x} \varphi\right]_{y^{\prime}}\right\}
$$

where $W_{i}$ decay exponentially with $\left|y-y^{\prime}\right|$. Crucially, $\mathcal{L}_{b}^{\prime}$ is short ranged in both parton and spin variables and thus does not affect the structure of the gauge theory. Performing the Gaussian integrals over $a_{\mu}$ and expressing $\varphi, \theta$ in terms of $\Phi, \Theta$ using Eq. (28), we find $\mathcal{S}=\int_{x, \tau} \sum_{y}\left[\mathcal{L}_{\text {spins }}+\mathcal{L}_{\text {spins }}^{\prime}\right]$ with

$$
\mathcal{L}_{\text {spins }}=\frac{i}{\pi} \partial_{x} \Theta_{y} \partial_{\tau} \Phi_{y}+\frac{v K}{2 \pi}\left(\partial_{x} \Phi_{y}\right)^{2}+\frac{v}{2 \pi K}\left(\partial_{x} \Theta_{y}\right)^{2}+\frac{u_{B}}{2 \pi}\left[S \partial_{x} \Theta\right]_{\tilde{y}}^{2},
$$

where $K=\frac{\kappa}{2} \sqrt{\frac{v_{B}}{v_{B}+2 \kappa \tilde{v}}}$ and $v=2 \tilde{v} K / \kappa$. The term $\mathcal{L}_{\text {spins }}^{\prime}$ contains exponentially decaying interwire interactions, i.e.,

$$
\mathcal{L}_{\text {spins }}^{\prime}=\frac{v^{2} K^{2}}{2 \pi v_{B}} \sum_{\tilde{y}^{\prime}}\left[\Delta \partial_{x} \Phi\right]_{\tilde{y}}\left[1-\frac{v K}{v_{B}} \Delta^{T} \Delta\right]_{\tilde{y}, \tilde{y}^{\prime}}^{-1}\left[\Delta \partial_{x} \Phi\right]_{\tilde{y}^{\prime}}+\sum_{y^{\prime}} \partial_{x} \Phi_{y}\left(P W_{2} P\right)_{y, y^{\prime}} \partial_{x} \Phi_{y^{\prime}}+\sum_{y^{\prime}}\left[S^{T} S \partial_{x} \Theta\right]_{y}\left(P W_{1} P\right)_{y, y^{\prime}}\left[S^{T} S \partial_{x} \Theta\right]_{y^{\prime}},
$$

where $P_{y, y^{\prime}}=(-1)^{y} \delta_{y, y^{\prime}}$. Since $\frac{v K}{v_{B}}<\frac{1}{4}$ for any choice of parameters, all terms in $\mathcal{L}_{\text {spins }}^{\prime}$ decay exponentially with $\left|y-y^{\prime}\right|$; a suitable choice of $W_{i}$ in Eq. (B3) can thus be used to achieve $\mathcal{L}_{\text {spins }}^{\prime}=0$. Alternatively, $W_{i}$ can be chosen such that $\mathcal{L}_{\text {spins }}^{\prime}=$ $\frac{u_{V}}{8 \pi}\left[\Delta \partial_{x} \Phi\right]_{\tilde{y}}^{2}$, which is the result stated in the main text, i.e., Eqs. (12) and (32).

\section{External probing field}

Before concluding this Appendix, we include the external probing field $\vec{A}$ that minimally couples to the conserved $S^{z}$ of the microscopic spins. Translating $\mathcal{L}_{A}$ of Eq. (39) to bosonic-parton variables we find

$$
\mathcal{L}_{A}=\frac{i}{\pi}(-1)^{\tilde{y}} \partial_{x} \theta_{\tilde{y}}\left[S^{-T} A_{0}\right]_{\tilde{y}}+\frac{v K}{\pi}(-1)^{\tilde{y}} \partial_{x} \varphi_{\tilde{y}}\left[S A_{1}\right]_{\tilde{y}}+\frac{v K}{2 \pi} A_{1, y}^{2},
$$

where $S_{\tilde{y}, y}^{-T}$ is the inverse transpose of $S_{\tilde{y}, y}$. To cast the coupling into a more revealing form, we shift $a_{\mu}$ according to

$$
\begin{aligned}
& a_{0, \tilde{y}} \rightarrow a_{0, \tilde{y}}-\frac{1}{4}(-1)^{\tilde{y}}\left[S A_{0}\right]_{\tilde{y}}+(-1)^{\tilde{y}}\left[S^{-T} A_{0}\right]_{\tilde{y}}, \\
& a_{1, \tilde{y}} \rightarrow a_{1, \tilde{y}}-\left(\frac{1}{4}-\frac{v K}{v_{B}}\right)(-1)^{\tilde{y}}\left[S A_{1}\right]_{\tilde{y}} .
\end{aligned}
$$

This shift completely cancels $\mathcal{L}_{A}$; the external probing field instead appears in $\mathcal{L}_{b}$ of Eq. (B1) through the replacement $a_{\mu, \tilde{y}} \rightarrow$ $a_{\mu, \tilde{y}}-\frac{1}{4}(-1)^{\tilde{y}}\left[S A_{\mu}\right]_{\tilde{y}}$ and in higher-order couplings to the emergent gauge field given by

$$
\mathcal{L}_{a-A}=\frac{K}{\pi v}(-1)^{y}\left[\Delta^{T} \Delta A_{0}\right]_{y}\left[\Delta a_{0}\right]_{y}+\frac{v K}{4 \pi}(-1)^{y}\left[\Delta^{T} \Delta A_{1}\right]_{y}\left[\Delta a_{1}\right]_{y} .
$$

\section{APPENDIX C: DERIVATION OF THE FERMIONIC-PARTON FIELD THEORY}

To derive the gauge theory for the fermionic partons, we follow the same steps as for the bosonic partons. Specifically, we consider the action $\mathcal{S}=\int_{x, \tau} \sum_{y}\left[\mathcal{L}_{f}+\mathcal{L}_{\text {Maxwell }}+\mathcal{L}_{f}^{\prime}+\mathcal{L}^{\prime}\right]$ with

$$
\begin{aligned}
\mathcal{L}_{f} & =\frac{i}{\pi} \partial_{x} \theta_{f, \tilde{y}}\left(\partial_{\tau} \varphi_{f, \tilde{y}}-a_{0, \tilde{y}}\right)+\frac{v_{F}}{2 \pi}\left(\partial_{x} \varphi_{f, \tilde{y}}-a_{1, \tilde{y}}\right)^{2}+\frac{u_{F}}{2 \pi}\left(\partial_{x} \theta_{f, \tilde{y}}\right)^{2}, \\
\mathcal{L}_{\text {Maxwell }} & =\frac{\kappa}{8 \pi \tilde{v}}\left[\Delta a_{0}\right]_{y}^{2}+\frac{\kappa \tilde{v}}{8 \pi}\left[\Delta a_{1}\right]_{y}^{2} .
\end{aligned}
$$

The first "primed" term $\mathcal{L}_{f}^{\prime}$ contains interwire interactions between fermion densities and currents, that decay exponentially with the wire separation $\left|y-y^{\prime}\right|$. This term is not essential for understanding the structure of the gauge theory. For completeness, we still provide an explicit treatment below. The final term is

$$
\mathcal{L}^{\prime}=\frac{i}{4 \pi} \alpha(-1)^{y}\left[\Delta a_{1}\right]_{y}\left[\Delta a_{0}\right]_{y} .
$$

It has an alternating sign and thus does not affect the long-distance behavior of the photon propagator (see Appendix D 3 below). However, as we will see, it is essential for the microscopic time-reversal symmetry of the spin system. To understand its origin, recall that the fermionic partons are obtained by attaching fluxes of opposite sign to the two bosonic-parton species. In the schematic continuum manipulations described by Eq. (73), the two Chern-Simons terms that implement this flux attachment 
exactly cancel. By contrast, in the wire regularization, the two species of fermionic partons reside at different locations (even vs odd dual wires). Consequently, the cancellation is imperfect, and a residual term $\mathcal{L}_{\mathrm{CS} \text {,even }}+\mathcal{L}_{\mathrm{CS} \text {,odd }} \propto \mathcal{L}^{\prime}+(-1)^{\tilde{y}} O\left(\Delta^{4}\right)$ remains.

To map the gauge theory to spin variables, we perform the Gaussian integrals over $a_{\mu}$ and express $\varphi_{f}, \theta_{f}$ in terms of $\Phi, \Theta$ using

$$
\begin{aligned}
\varphi_{f, \tilde{y}} & =-(-1)^{\tilde{y}}\left[S^{-T} \Phi\right]_{\tilde{y}}+2\left[\Delta^{-T} \Theta\right]_{\tilde{y}}-[\Delta \Theta]_{\tilde{y}}, \\
\theta_{f, \tilde{y}} & =-(-1)^{\tilde{y}}[S \Theta]_{\tilde{y}},
\end{aligned}
$$

with $\left(\Delta^{-T}\right)_{\tilde{y}, y^{\prime}}$ the inverse transpose of $\Delta_{\tilde{y}, y^{\prime}}$. We find $\mathcal{S}=\int_{x, \tau} \sum_{y}\left[\mathcal{L}_{\text {spins }}+\mathcal{L}_{\text {spins }}^{\prime}+\mathcal{L}_{\text {spins }}^{\prime \prime}\right]$, where

$$
\mathcal{L}_{\text {spins }}=\frac{i}{\pi} \partial_{x} \Theta_{y} \partial_{\tau} \Phi_{y}+\frac{v K}{2 \pi}\left(\partial_{x} \Phi_{y}\right)^{2}+\frac{v}{2 \pi K}\left(\partial_{x} \Theta_{y}\right)^{2}+\frac{u_{V}}{4 \pi}\left[\Delta \partial_{x} \Phi\right]_{\tilde{y}}+\frac{\overline{u_{F}}}{2 \pi}\left[S \partial_{x} \Theta\right]_{\tilde{y}}^{2},
$$

with parameters $K=\frac{\alpha}{2 \kappa} \sqrt{\frac{\kappa v_{F}}{\kappa v_{F}+\left(\alpha^{2}+\kappa^{2}\right) \tilde{v}}}, v=2 \tilde{v} \kappa K / \alpha, u_{V}=v_{F}\left(\frac{\alpha \tilde{v}}{2 \alpha \tilde{v}+\kappa v_{F}}\right)^{2}$, and $\overline{u_{F}}=u_{F}+4 v K$. The term $\mathcal{L}_{\text {spins }}^{\prime}$ contains exponentially decaying interwire terms whose explicit form is provided below. Crucially, a suitable choice of the $\mathcal{L}_{\text {parton }}^{\prime}$ results in $\mathcal{L}_{\text {spins }}^{\prime}=0$, and can additionally be used to tune the parameters $u_{V}$ and $\overline{u_{F}}$ in Eq. (C6). The final term

$$
\mathcal{L}_{\text {spins }}^{\prime \prime}=\frac{\bar{v}}{2 \pi}(-1)^{y} \partial_{x} \Theta_{y} \partial_{x} \Phi_{y},
$$

with $\bar{v}=4 v K\left(\frac{2 \alpha}{\alpha^{2}+\kappa^{2}}-1\right)$, cannot be eliminated by any choice of $\mathcal{L}_{\text {parton }}^{\prime}$, which contains only higher orders in $\Delta$. Moreover, it violates the microscopic time-reversal symmetry. To preserve this symmetry, we must, therefore, choose $\alpha$ in the gauge theory of Eq. (C3) such that $\bar{v}=0$, namely, $\alpha=1 \pm \sqrt{1-\kappa^{2}} \equiv \alpha_{0}$. Having eliminated $\mathcal{L}_{\text {spins }}^{\prime}$ and $\mathcal{L}_{\text {spins }}^{\prime \prime}$, the action matches Eqs. $(12)$ and (32) with the parameters specified above.

\section{Explicit form of short-range interactions}

We include generic short-range density-density, current-current, and density-current interactions for the fermionic parton in the form

$$
\mathcal{L}_{\text {parton }}^{\prime}=\sum_{\tilde{y}^{\prime}}\left\{\left[\Delta \partial_{x} \theta_{f}\right]_{y}\left(W_{1}\right)_{y, y^{\prime}}\left[\Delta \partial_{x} \theta_{f}\right]_{y^{\prime}}+\left[S^{T} \Delta \partial_{x} \varphi_{f}\right]_{\tilde{y}}\left(W_{2}\right)_{\tilde{y}, \tilde{y}^{\prime}}\left[S^{T} \Delta \partial_{x} \varphi_{f}\right]_{\tilde{y}^{\prime}}+\left[\Delta \partial_{x} \theta_{f}\right]_{y}\left(W_{3}\right)_{y, y^{\prime}}\left[P \Delta \partial_{x} \varphi_{f}\right]_{y^{\prime}}\right\},
$$

where $W_{i}$ decay exponentially with $\left|y-y^{\prime}\right|$. It is straightforward to express these in terms of $\Phi, \Theta$ and combine them with the exponentially decaying interactions stemming from the Gaussian integrals over $a_{\mu}$. We find

$$
\mathcal{L}_{\text {spin }}^{\prime}=\frac{v_{F}}{2 \pi} \sum_{\tilde{y}^{\prime}}\left\{\left[S \partial_{x} \Theta\right]_{\tilde{y}}\left(\widetilde{W}_{1}\right)_{\tilde{y}, \tilde{y}^{\prime}}\left[S \partial_{x} \Theta\right]_{\tilde{y}^{\prime}}+\left[\Delta \partial_{x} \Phi\right]_{\tilde{y}}\left(\widetilde{W}_{2}\right)_{\tilde{y}, \tilde{y}^{\prime}}\left[\Delta \partial_{x} \Phi\right]_{\tilde{y}^{\prime}}-\left[S \partial_{x} \Theta\right]_{\tilde{y}}\left(\widetilde{W}_{3}\right)_{\tilde{y}, \tilde{y}^{\prime}}\left[P \Delta \partial_{x} \Phi\right]_{\tilde{y}^{\prime}}\right\}
$$

with exponentially decaying $\widetilde{W}_{i}$. We are interested in $\bar{v}=0$, for which these kernels are given by

$$
\begin{aligned}
& \widetilde{W}_{1}=S\left(V_{2}-\Delta^{T} W_{2} \Delta+P W_{1} P+P W_{3} P\right) S^{T}+4 W_{2}-2 P W_{3} P-4 g, \\
& \widetilde{W}_{2}=V_{1}+P W_{2} P, \\
& \widetilde{W}_{3}=2\left[V_{2}+\left(2-\Delta^{T} \Delta\right) W_{2}+\frac{1}{2} P W_{3} P\right],
\end{aligned}
$$

where $g=\frac{v K}{v_{F}}, V_{1}=g^{3} \Delta^{T}\left[1-g \Delta^{T} \Delta\right]^{-1} \Delta$, and $V_{2}=\frac{g}{1-4 g}\left[1+\frac{g}{1-4 g} \Delta^{T} \Delta\right]^{-1}$. A choice of exponentially decaying $W_{i}$ that results in $\mathcal{L}_{\text {spins }}^{\prime}=0$ is given by

$$
\begin{aligned}
& W_{1}=2 g\left(1-2 g-2 g^{2}+g^{2} \Delta^{T} \Delta-\frac{1}{2} g^{2} \Delta^{T} \Delta \Delta^{T} \Delta\right)\left[1-g \Delta^{T} \Delta\right]^{-1}, \\
& W_{2}=-\frac{g^{3}}{1-4 g} S^{T} S\left[1+\frac{g}{1-4 g} \Delta^{T} \Delta\right]^{-1}, \\
& W_{3}=-2 g\left[1+g^{2} \Delta^{T} \Delta\left(2-\Delta^{T} \Delta\right)\right]\left[1-g \Delta^{T} \Delta\right]^{-1} .
\end{aligned}
$$

\section{External probing field}

Before concluding the derivation, we also include an external probing field $\vec{A}$ that minimally couples to the conserved $S^{z}$ of the microscopic spins. We thus supplement the action of the gauge theory action by $\mathcal{L}_{A}$ as given in Eq. (39). In terms of the fermionic-parton variables

$$
\mathcal{L}_{A}=\frac{i}{\pi}(-1)^{\tilde{y}} \partial_{x} \theta_{f, \tilde{y}}\left[S^{-T} A_{0}\right]_{\tilde{y}}+\frac{v K}{\pi}(-1)^{\tilde{y}} \partial_{x} \varphi_{f, \tilde{y}}\left[S A_{1}\right]_{\tilde{y}}-\frac{v K}{\pi} \partial_{x} \theta_{f, \tilde{y}}\left[\left(2-\Delta^{T} \Delta\right) \Delta^{-T} A_{1}\right]_{\tilde{y}}+\frac{v K}{2 \pi} A_{1, y}^{2} .
$$


To cast this into a more revealing form, we shift $a_{\mu}$ according to

$$
\begin{aligned}
& a_{0, \tilde{y}} \rightarrow a_{0, \tilde{y}}-\frac{1}{4}(-1)^{\tilde{y}}\left[S A_{0}\right]_{\tilde{y}}+(-1)^{\tilde{y}}\left[S^{-T} A_{0}\right]_{\tilde{y}}+i v K\left[\left(2-\Delta^{T} \Delta\right) \Delta^{-T} A_{1}\right]_{\tilde{y}}, \\
& a_{1, \tilde{y}} \rightarrow a_{1, \tilde{y}}-\left(\frac{1}{4}-\frac{v K}{v_{F}}\right)(-1)^{\tilde{y}}\left[S A_{1}\right]_{\tilde{y}} .
\end{aligned}
$$

This completely cancels $\mathcal{L}_{A}$; the external probing field instead appears in $\mathcal{L}_{f}$ of Eq. (C1) through the replacement $a_{\mu, \tilde{y}} \rightarrow$ $a_{\mu, \tilde{y}}-\frac{1}{4}(-1)^{\tilde{y}}\left[S A_{\mu}\right]_{\tilde{y}}$ and in higher-order couplings to the emergent gauge field given by

$$
\begin{aligned}
\mathcal{L}_{a-A}= & -\frac{K(2-\alpha)(-1)^{y}}{8 \pi v}\left[\Delta^{T} a_{0}\right]_{y}\left[\Delta^{T} \Delta A_{0}\right]_{y}-\frac{v K(2+\alpha)(-1)^{y}}{8 \pi}\left[\Delta^{T} a_{1}\right]_{y}\left[\Delta^{T} \Delta A_{1}\right]_{y} \\
& -\frac{i K^{2}}{4 \pi}\left[\Delta^{T} a_{0}\right]_{y}\left[\Delta^{T} \Delta A_{1}\right]_{y}+\frac{i \alpha}{16 \pi}\left[\Delta^{T} a_{1}\right]_{y}\left[\Delta^{T} \Delta A_{0}\right]_{y} .
\end{aligned}
$$

\section{APPENDIX D: GAUGE-THEORY CALCULATIONS}

\section{Bosonic-parton superfluid}

To analyze the implications of a condensed bosonic parton, first recall that the gauge theory is of the form $\mathcal{L}=$ $\sum_{\sigma} \mathcal{L}_{\sigma}\left[b_{\sigma}, a_{\sigma}+\frac{\sigma}{2} \mathrm{~A}_{\sigma}\right]+\mathcal{L}_{\text {Maxwell }}\left[a_{\uparrow}, a_{\downarrow}\right]$, where $\overrightarrow{\mathrm{A}}_{\tilde{y}} \equiv\left(\vec{A}_{\tilde{y}-1 / 2}+\vec{A}_{\tilde{y}+1 / 2}\right) / 2$ and we have endowed $\vec{a}$ and $\overrightarrow{\mathrm{A}}$ with the (redundant) spin label corresponding to the dual-wire parity. The two species couple only through the Maxwell term

$$
\mathcal{L}_{\text {Maxwell }}=\frac{\kappa}{4 \pi}\left\{\frac{1}{\tilde{v}}\left[\left(a_{0,2 \tilde{y}, \downarrow}-a_{0,2 \tilde{y}-1, \uparrow}\right)^{2}+\left(a_{0,2 \tilde{y}+1, \uparrow}-a_{0,2 \tilde{y}, \downarrow}\right)^{2}\right]+\tilde{v}\left[\left(a_{1,2 \tilde{y}, \downarrow}-a_{1,2 \tilde{y}-1, \uparrow}\right)^{2}+\left(a_{1,2 \tilde{y}+1, \uparrow}-a_{1,2 \tilde{y}, \downarrow}\right)^{2}\right]\right\} .
$$

(Additional short-range interactions between opposite species can always be subsumed by adding higher-order derivatives to the Maxwell term.) Condensation of $b_{\downarrow}$ corresponds to $\delta \mathcal{L}_{\downarrow}=g_{t} \cos \left(\varphi_{2 \tilde{y}+2, \downarrow}-\varphi_{2 \tilde{y}, \downarrow}\right)$ reaching strong coupling first. We introduce a dimensionless coupling constant with bare value $\tilde{g}_{t}=\pi d_{0}^{2} g_{t} / v_{B}$ and replace

$$
\delta \mathcal{L}_{\downarrow}=\frac{v_{B} \tilde{g}_{t}}{\pi d_{0}^{2}} \cos \left(\left[\Delta_{2} \varphi_{\downarrow}\right]_{2 \tilde{y}+1}\right) \rightarrow \frac{v_{B}}{2 \pi d_{*}^{2}}\left[\Delta_{2} \varphi_{\downarrow}\right]_{2 \tilde{y}+1}^{2} .
$$

Here, $d_{*}$ is the length scale at which the coupling constant renormalized to order unity. Upon integrating out $\theta_{\downarrow}$ the Lagrangian governing $\varphi_{\downarrow}$ is given by

$$
\mathcal{L}_{\downarrow}+\delta \mathcal{L}_{\downarrow}=\frac{1}{2 \pi u_{B}}\left|i \omega \varphi_{\downarrow}-a_{0, \downarrow}+\frac{1}{2} \mathrm{~A}_{0, \downarrow}\right|^{2}+\frac{v_{B}}{2 \pi}\left|i k_{x} \varphi_{\downarrow}-a_{1, \downarrow}+\frac{1}{2} \mathrm{~A}_{1, \downarrow}\right|^{2}+\frac{v_{B}}{2 \pi}\left|\Delta_{k_{y}} \varphi_{\downarrow}\right|^{2},
$$

where we have Fourier transformed using a two-wire unit cell. Further performing the Gaussian integral over $\varphi_{\downarrow}$ we obtain $\mathcal{L}_{\text {ind }}\left[a_{\downarrow}-\frac{1}{2} \mathbf{A}_{\downarrow}\right]=\frac{1}{2 \pi}\left(a_{\downarrow}^{*}-\frac{1}{2} \mathbf{A}_{\downarrow}^{*}\right)_{\mu} \Pi_{\mu \nu}^{\text {ind }}\left(a_{\downarrow}-\frac{1}{2} \mathbf{A}_{\downarrow}\right)_{\nu}$ with

$$
\Pi^{\text {ind }}=\frac{1}{\omega^{2}+u_{B} v_{B} k_{x}^{2}+u_{B} v_{B}\left|\Delta_{k_{y}}\right|^{2}}\left(\begin{array}{cc}
v_{B} k_{x}^{2}+v_{B}\left|\Delta_{k_{y}}\right|^{2} & -v_{B} \omega k_{x} \\
-v_{B} \omega k_{x} & v_{B} \omega^{2}+u_{B} v_{B}^{2}\left|\Delta_{k_{y}}\right|^{2}
\end{array}\right) .
$$

This induced action supplements the bare $\mathcal{L}_{\text {Maxwell }}$, which in Fourier space reads as

$$
\mathcal{L}_{\text {Maxwell }}=\frac{\kappa}{4 \pi}\left[\frac{1}{\tilde{v}}\left(\left|a_{0, \downarrow}-e^{-i d_{0} k_{y}} a_{0, \uparrow}\right|^{2}+\left|a_{0, \uparrow}-a_{0, \downarrow}\right|^{2}\right)+\tilde{v}\left(\left|a_{1, \downarrow}-e^{-i d_{0} k_{y}} a_{1, \uparrow}\right|^{2}+\left|a_{1, \uparrow}-a_{1, \downarrow}\right|^{2}\right)\right] .
$$

We now shift $a_{\mu, \sigma} \rightarrow a_{\mu, \sigma}+\frac{1}{2} \mathrm{~A}_{\mu, \downarrow}$ such that $\mathrm{A}_{\mu, \downarrow}$ is eliminated from $\mathcal{L}_{\text {ind }}$. It instead enters $\mathcal{L}_{\uparrow}$ and, at subleading order $\left|\Delta_{k_{y}}\right|^{2} \mathbf{A}_{\mu, \downarrow}$, the Maxwell term $\mathcal{L}_{\text {Maxwell }}$. Finally, performing the integral over $a_{\mu, \downarrow}$ and retaining only the leading orders in $\Delta_{k_{y}}$ we arrive at the effective action $\mathcal{L}_{\text {eff }}=\mathcal{L}_{\uparrow}\left[b_{\uparrow}, a_{\uparrow}+\frac{\mathrm{A}_{\uparrow}+\mathrm{A}_{\downarrow}}{2}\right]+\frac{1}{2 \pi} \gamma_{\mu} a_{\mu, \uparrow}^{*}\left[\Pi^{(0)}+\Pi^{(2)}\right]_{\mu \nu} \gamma_{\nu} a_{\nu, \uparrow}$ with

$$
\begin{aligned}
\Pi^{(0)} & =\frac{1}{\omega^{2}+c_{x}^{2} k_{x}^{2}+c_{y}^{2}\left|\Delta_{k_{y}}\right|^{2}}\left(\begin{array}{cc}
c_{x}^{2} k_{x}^{2}+c_{y}^{2}\left|\Delta_{k_{y}}\right|^{2} & -c_{x} k_{x} \omega \\
-c_{x} k_{x} \omega & c_{y}^{2}\left|\Delta_{k_{y}}\right|^{2}+\omega^{2}
\end{array}\right), \\
\Pi^{(2)} & =\frac{\left|\Delta_{k_{y}}\right|^{2}}{4\left[\omega^{2}+c_{1}^{2} k_{x}^{2}+c_{2}^{2}\left|\Delta_{k_{y}}\right|^{2}\right]}\left(\begin{array}{cc}
\frac{\kappa u_{B}}{\tilde{v}}\left[c_{1}^{2} k_{x}^{2}+c_{2}^{2}\left|\Delta_{k_{y}}\right|^{2}+\frac{\tilde{u}_{B}+\tilde{v}}{\kappa u_{B}} \omega^{2}\right] & c_{1} k_{x} \omega \\
c_{1} k_{x} \omega & \frac{\kappa \tilde{v}}{v_{B}}\left[\frac{v_{B}+\kappa \tilde{v}}{\kappa \tilde{v}} c_{1}^{2} k_{x}^{2}+c_{2}^{2}\left|\Delta_{k_{y}}\right|^{2}+\omega^{2}\right]
\end{array}\right),
\end{aligned}
$$

where $c_{x}^{2}=\frac{v_{B} \tilde{v}\left(\kappa u_{B}+\tilde{v}\right)}{v_{B}+\kappa \tilde{v}}, \gamma_{0}=\sqrt{\frac{\kappa}{\left(\kappa u_{B}+\tilde{v}\right)}}, c_{y}^{2}=\frac{v_{B}}{\gamma_{0}^{2}}$, and $\gamma_{1}=\gamma_{0} c_{x}$. The analytically continued gauge-field propagator has poles at real frequencies $\omega= \pm \sqrt{\tilde{v}^{2} k_{x}^{2}+v_{B} u_{B}\left|\Delta_{k_{y}}\right|^{2}+\frac{4 v_{B} \tilde{v}}{\kappa d_{*}^{2}}}$. For any finite $d_{*}$, the gauge field $\vec{a}_{\uparrow}$ is gapped; integrating it out results in 
$\mathcal{L}_{\uparrow}\left[b_{\uparrow}, \frac{1}{2} \mathrm{~A}_{\uparrow}+\frac{1}{2} \mathrm{~A}_{\downarrow}\right]$, up to renormalization of parameters and short-range interactions. Crucially, $b_{\uparrow}$ now couples to the external probing field with unit charge, i.e., carries spin 1.

Finally, consider the case where the second parton also condenses, i.e., where $\delta \mathcal{L}_{\uparrow} \sim \cos \left(\varphi_{2 \tilde{y}+1, \uparrow}-\varphi_{2 \tilde{y}-1, \uparrow}\right)$ flows to strong coupling as well. Treating $\delta \mathcal{L}_{\uparrow}$ in the same way as $\delta \mathcal{L}_{\downarrow}$ and integrating out $b_{\uparrow}$, we find

$$
\mathcal{L}_{\text {Meissner }}=\frac{v_{B}}{2 \pi} \bar{A}_{\mu}\left[\delta^{\mu \nu}-\frac{p^{\mu} p^{\nu}}{\vec{p} \cdot \vec{p}}\right] \bar{A}_{\nu}+O\left(p^{2}\right),
$$

where $\vec{A}=\left(A_{0} \frac{1}{\sqrt{u_{B} v_{B}}}, \boldsymbol{A}\right)$ and $\vec{p}=\left(\frac{\omega}{\sqrt{v_{B} u_{B}}}, k_{x}, k_{y}\right)$. This response to the probing field $\vec{A}$ implies that the U(1) spin-rotation symmetry is spontaneously broken.

\section{Bosonic-parton quantum Hall state}

The Lagrangian for the quantum Hall state of bosonic partons, described in Sec. IV B 4, is

$$
\mathcal{L}_{\mathrm{QH}}=\frac{i}{\pi} \partial_{x} \theta_{\tilde{y}}\left(\partial_{\tau} \varphi_{\tilde{y}}-a_{0, \tilde{y}}\right)+\frac{v_{B}}{2 \pi}\left(\partial_{x} \varphi_{\tilde{y}}-a_{1, \tilde{y}}\right)^{2}+\frac{u_{B}}{2 \pi}\left(\partial_{x} \theta_{\tilde{y}}\right)^{2}+g_{\mathrm{QH}} \cos \left(S^{T} \Delta \varphi_{\tilde{y}}-2 n \theta_{\tilde{y}}\right)+\mathcal{L}_{\text {Maxwell }},
$$

with $\mathcal{L}_{\text {Maxwell }}=\frac{\kappa}{4 \pi \tilde{v}}\left[\Delta a_{0}\right]_{y}^{2}+\frac{\kappa \tilde{v}}{4 \pi}\left[\Delta a_{1}\right]_{y}^{2}$. To integrate out the bosonic partons, we introduce new conjugate variables that diagonalize the interwire interaction, i.e., $\bar{\theta}_{\tilde{y}}=\frac{1}{2}\left(S^{T} \Delta \varphi_{\tilde{y}}\right)+n \theta_{\tilde{y}}$ and $\bar{\varphi}_{\tilde{y}}=\frac{1}{n} \varphi_{\tilde{y}}$. In terms of $\bar{\theta}$ and $\bar{\varphi}$, the Lagrangian is

$$
\begin{aligned}
\mathcal{L}_{\mathrm{QH}}= & \frac{i}{\pi} \partial_{x} \bar{\theta}_{\tilde{y}}\left(\partial_{\tau} \bar{\varphi}_{\tilde{y}}-a_{0, \tilde{y}}\right)+\frac{v_{B}}{2 \pi}\left(n \partial_{x} \bar{\varphi}_{\tilde{y}}-a_{1, \tilde{y}}\right)^{2}+\frac{u_{B}}{2 \pi n^{2}}\left(\partial_{x} \bar{\theta}_{\tilde{y}}\right)^{2}+g_{\mathrm{QH}} \cos \left(2 \bar{\theta}_{\tilde{y}}\right) \\
& +\frac{i}{2 \pi} S^{T} \Delta \partial_{x} \bar{\varphi}_{\tilde{y}} a_{0, \tilde{y}}+\frac{u_{B}}{8 \pi}\left(S^{T} \Delta \partial_{x} \bar{\varphi}_{\tilde{y}}\right)^{2}-\frac{u_{B}}{2 \pi n} \partial_{x} \bar{\theta}_{\tilde{y}} S^{T} \Delta \partial_{x} \bar{\varphi}_{\tilde{y}}+\mathcal{L}_{\text {Maxwell }} .
\end{aligned}
$$

We introduce the dimensionless coupling constant $\tilde{g}_{\mathrm{QH}}=8 \pi d_{0}^{2} g_{\mathrm{QH}} / v_{B}$ and replace $\frac{v_{B} \tilde{g}_{\mathrm{QH}}}{8 \pi d_{0}^{2}} \cos \left(2 \bar{\theta}_{\tilde{y}}\right) \rightarrow \frac{v_{B}}{2 \pi d_{*}^{2}} \bar{\theta}_{\tilde{y}}^{2}$, where $d_{*}$ is the length scale at which it renormalizes to order unity. Performing the Gaussian integral over $\bar{\varphi}$ we find, to lowest order in derivatives,

$$
\mathcal{L}_{\mathrm{QH}}^{\prime}=\frac{i}{\pi n} \bar{\theta}_{\tilde{y}}\left(\partial_{x} a_{0, \tilde{y}}-\partial_{\tau} a_{1, \tilde{y}}\right)+\frac{v_{B}}{2 \pi d_{*}^{2}} \bar{\theta}_{\tilde{y}}^{2}+\frac{i}{2 \pi n}\left[S a_{1}\right]_{y}\left[\Delta a_{0}\right]_{y}+\frac{1}{2 \pi v_{B} n^{2}}\left[\Delta a_{0}\right]_{y}^{2}+\frac{u_{B}}{2 \pi n^{2}}\left[\Delta a_{1}\right]_{y}^{2} .
$$

Finally, we perform the integral over $\bar{\theta}$ and obtain the effective gauge theory

$$
\mathcal{L}_{\text {gauge }}=\frac{i}{2 \pi n}\left[S a_{1}\right]_{y}\left[\Delta a_{0}\right]_{y}+\left(\frac{\kappa}{4 \pi \tilde{v}}+\frac{1}{2 \pi v_{B} n^{2}}\right)\left[\Delta a_{0}\right]_{y}^{2}+\left(\frac{\kappa \tilde{v}}{4 \pi}+\frac{u_{B}}{2 \pi n^{2}}\right)\left[\Delta a_{1}\right]_{y}^{2}+\frac{d_{*}^{2}}{2 \pi v_{B} n^{2}}\left(\partial_{x} a_{0, \tilde{y}}-\partial_{\tau} a_{1, \tilde{y}}\right)^{2} .
$$

\section{Fermionic-parton QSH}

To assess the relevance of monopoles in the QSH phase of fermionic partons we compute their correlation function within the monopole-free theory. The corresponding Lagrangian was introduced in Sec. V B 2; it is given by $\mathcal{L}_{\mathrm{QSH}}=\mathcal{L}_{\text {matter }}+\mathcal{L}_{\text {gauge }}$ with

$$
\begin{aligned}
\mathcal{L}_{\text {matter }} & =\frac{i}{\pi} \partial_{x} \theta_{f, \tilde{y}, \sigma}\left(\partial_{\tau} \varphi_{f, \tilde{y}, \sigma}-a_{0, \tilde{y}}\right)+\frac{v_{F}}{2 \pi}\left(\partial_{x} \varphi_{f, \tilde{y}, \sigma}-a_{1, \tilde{y}}\right)^{2}+\frac{u_{F}}{2 \pi}\left(\partial_{x} \theta_{f, \tilde{y}, \sigma}\right)^{2}+\frac{\tilde{v}}{8 \pi \kappa d_{*}^{2}}\left[S_{2} \theta_{f, \sigma}-P \Delta_{2} \varphi_{f, \sigma}\right]_{\tilde{y}}^{2}, \\
\mathcal{L}_{\text {gauge }} & =\frac{\kappa}{8 \pi \tilde{v}}\left[\Delta a_{0}\right]_{y}^{2}+\frac{\kappa \tilde{v}}{8 \pi}\left[\Delta a_{1}\right]_{y}^{2}+\frac{i}{4 \pi} \alpha(-1)^{y}\left[\Delta a_{1}\right]_{y}\left[\Delta a_{0}\right]_{y},
\end{aligned}
$$

where, as usual, $d_{*}$ is the length scale beyond which the quadratic approximation of the cosine is appropriate. (For the last term in $\mathcal{L}_{\text {gauge }}$, cf. Appendix C.) It is straightforward to integrate out the matter fields and obtain the induced gauge-field action. Upon expanding in frequencies and $x$ momenta small compared to the QSH gap, $E_{\mathrm{QSH}} \equiv \sqrt{\frac{4 \tilde{v} v_{F}}{\kappa d_{*}^{2}}}$, we find

$$
\mathcal{L}_{\text {ind }}=\frac{1}{8 \pi v_{F}}\left[\Delta_{2} a_{0}\right]_{\tilde{y}}^{2}+\frac{u_{F}}{8 \pi}\left[\Delta_{2} a_{1}\right]_{\tilde{y}}^{2}+\frac{\kappa d_{*}^{2}}{8 \pi \tilde{v}}\left(\partial_{x} a_{0, \tilde{y}}-\partial_{\tau} a_{1, \tilde{y}}\right)^{2}+\frac{i}{4 \pi}(-1)^{\tilde{y}}\left[S_{2} a_{1}\right]_{\tilde{y}}\left[\Delta_{2} a_{0}\right]_{\tilde{y}}+\mathcal{L}_{\text {ind }}^{\prime} .
$$

Here $\mathcal{L}_{\text {ind }}^{\prime}$ contains terms suppressed by at least two additional powers of $\Delta_{2}$. These contributions can easily be retained but do not affect any long-wavelength properties; we, therefore, discard them. In the absence of oscillatory terms $\sim(-1)^{\tilde{y}}$ in the total gauge-field action $\mathcal{L}_{\text {gauge }}+\mathcal{L}_{\text {ind }}$, we approximate $\Delta_{2}=2 \Delta+\Delta^{2} \approx 2 \Delta$ for describing long-wavelength properties. We thus obtain the effective gauge-field action

$$
\mathcal{L}_{\text {gauge }}^{\text {eff }}=\frac{\kappa}{8 \pi}\left\{\frac{1}{\tilde{v}} \gamma^{2}\left[\Delta a_{0}\right]_{y}^{2}+\tilde{v} \eta^{2}\left[\Delta a_{1}\right]_{y}^{2}+d_{*}^{2} \frac{1}{\tilde{v}}\left(\partial_{x} a_{0, \tilde{y}}-\partial_{\tau} a_{1, \tilde{y}}\right)^{2}\right\},
$$


where $\gamma^{2}=1+\frac{4 \tilde{v}}{\kappa v_{F}}$ and $\eta^{2}=1+\frac{4 u_{F}}{\kappa \tilde{v}}$. Oscillatory terms $\sim(-1)^{\tilde{y}}$ couple gauge fields at $y$ momenta $k_{y}$ and $k_{y}+\pi / d_{*}$. We obtain the long-wavelength action in that case, by integrating out modes at momenta $\left|k_{y}\right|>\pi / 2 d_{*}$. Returning to real space, we again find $\mathcal{L}_{\text {gauge }}^{\text {eff }}$, but with modified parameters $\gamma^{2}=\frac{\kappa^{2}+(2-\alpha)^{2}}{\kappa^{2}}+\frac{4 \tilde{v}}{\kappa v_{F}}, \eta^{2}=\frac{\kappa^{2}+(2+\alpha)^{2}}{\kappa^{2}}+\frac{4 u_{F}}{\kappa \tilde{v}}$.

To compute the correlation function of a probing monopole in this theory, we supplement $\mathcal{L}_{\text {gauge }}^{\text {eff }}$ by the monopole action of Eq. (82) and integrate out $a_{\mu}$. At leading order in $\Delta_{k_{y}}$, we find

$$
\mathcal{L}_{\mathcal{M}, \text { eff }}=\left[\frac{\tilde{v}}{2 \pi \kappa}+\frac{\tilde{v}}{2 \pi \kappa \gamma^{2}} \frac{\eta^{2} \tilde{v}^{2}\left|\Delta_{k_{y}}\right|^{2}+\omega^{2}}{\eta^{2} \tilde{v}^{2}\left|\Delta_{k_{y}}\right|^{2}+\omega^{2}+\frac{\eta^{2}}{\gamma^{2}} \tilde{v}^{2} k_{x}^{2}}\right] k_{x}^{2}\left|\phi_{\mathcal{M}}\right|^{2}+\frac{g_{\mathcal{M}}}{2}\left[\mathcal{M}_{-\vec{k}} \mathcal{M}_{\vec{k}}+\mathcal{M}_{-\vec{k}}^{\dagger} \mathcal{M}_{\vec{k}}^{\dagger}\right]
$$

The monopole-monopole correlation function, evaluated within a monopole-free background $g_{\mathcal{M}}=0$, is given by

$$
C_{\boldsymbol{R}}^{\mathcal{M}}=\exp \left\{-\frac{\kappa d_{*}}{8 \pi^{2} \tilde{v}} \frac{\gamma^{2}}{\gamma^{2}+1} \int_{\boldsymbol{k}, \omega}[2-2 \cos (\boldsymbol{k} \cdot \boldsymbol{R})]\left[\frac{1}{k_{x}^{2}}+O(1)\right]\right\} \begin{cases}e^{-\frac{4}{\pi} \frac{\gamma^{2}}{\gamma^{2}+1}\left(\frac{x}{d_{*}}\right)^{2}}, & y=0 \\ e^{-\frac{2}{\pi} \sqrt{\frac{\kappa \tilde{v}}{v_{F}}} \frac{\gamma^{2}}{\gamma^{2}+1} f(y) \frac{L}{d_{*}}}, & y \neq 0\end{cases}
$$

where $L$ is the wire length, and the $\omega$ and $k_{x}$ integrals are cut off by $E_{\mathrm{QSH}}$ and $v_{F}^{-1} E_{\mathrm{QSH}}$, respectively. The function $f(y) \equiv$ $1-\frac{\sin (\pi y / 2)}{\pi y / 2}$ is bounded from below by $1-2 / \pi \approx 0.36$ for nonzero $y$. At long distances, this correlation function decays faster than exponential. Monopoles described by $\mathcal{M}$ are thus strongly irrelevant.

We now turn to dressed monopoles, which were introduced in Sec. V B 2 via the shorthand $\mathcal{M}_{\text {dressed }} \sim f_{\downarrow}^{\dagger} f_{\uparrow} \mathcal{M}$. Their explicit expression is

$$
\mathcal{M}_{\text {dressed }, \boldsymbol{r}}= \begin{cases}\mathcal{M}_{\boldsymbol{r}} f_{\boldsymbol{r}-\frac{1}{2} \hat{y}, \uparrow, L}^{\dagger} f_{\boldsymbol{r}+\frac{1}{2} \hat{y}, \downarrow, L}, & y \text { even } \\ \mathcal{M}_{\boldsymbol{r}} f_{\boldsymbol{r}+\frac{1}{2} \hat{y}, \uparrow, R} f_{\boldsymbol{r}-\frac{1}{2} \hat{y}, \downarrow, R}, & y \text { odd. }\end{cases}
$$

It is instructive to relate its correlation function to configurations of the emergent gauge field $\vec{a}$. We therefore write

$$
C_{\mathrm{dressed}, \boldsymbol{r}-\boldsymbol{r}^{\prime}}^{\mathcal{M}}=\left\langle\mathcal{M}_{\mathrm{dressed}, \boldsymbol{r}}^{\dagger} \mathcal{M}_{\mathrm{dressed}, \boldsymbol{r}^{\prime}}\right\rangle \equiv \frac{\int \mathcal{D}\left[f_{\sigma}, a, \phi_{\mathcal{M}}\right] \mathcal{M}_{\mathrm{dressed}, \boldsymbol{r}}^{\dagger} \mathcal{M}_{\mathrm{dressed}, \boldsymbol{r}^{\prime}} e^{-\mathcal{S}_{\mathcal{M}}-\mathcal{S}_{\mathrm{QSH}}}}{\int \mathcal{D}\left[f_{\sigma}, a, \phi_{\mathcal{M}}\right] e^{-\mathcal{S}_{\mathcal{M}}-\mathcal{S}_{\mathrm{QSH}}}}
$$

and perform the integrals over both the fermions and the monopole field $\phi_{\mathcal{M}}$. To lowest order in $k_{x}$, we find

$$
C_{\text {dressed }, \boldsymbol{R}}^{\mathcal{M}}=\exp \left\{-\frac{\kappa d_{*}}{16 \pi^{2} \tilde{v}} \gamma^{2} \int_{\boldsymbol{k}, \omega} \frac{2-2 \cos (\boldsymbol{k} \cdot \boldsymbol{R})}{k_{x}^{2}}\left[1-\frac{\kappa}{4 \pi \tilde{v}} \gamma^{2} d_{*}^{2}\left\langle\left|\varepsilon_{2}\right|^{2}\right\rangle_{0}\right]\right\},
$$

where $\langle\ldots\rangle_{0}$ is evaluated with respect to Eq. (D16) and $\varepsilon_{2}=\Delta a_{0} / d_{*}$ is the $y$ component of the emergent electric field in the $a_{2}=0$ gauge. Consequently, the long-distance behavior of the dressed monopole-monopole correlation function matches the form obtained by evaluating Eq. (10) with the appropriate singular configuration $\vec{a}^{\mathcal{M}}$ (cf. Fig. 7 ). Explicitly, we find

$$
C_{\text {dressed, } \boldsymbol{R}}^{\mathcal{M}} \sim \exp \left[\frac{\kappa \eta d_{*}}{4 \sqrt{x^{2}+\left(\frac{d_{*} y}{\gamma}\right)^{2}}}\right] .
$$

We conclude by pointing out that $C^{\mathcal{M}}$ can also be expressed in the form of Eq. (D21) but with $\gamma^{2}$ replaced by $\gamma^{2}-\frac{4 \tilde{v}}{\kappa v_{F}}$. This apparently innocuous change spoils the cancellation between the bare $k_{x}^{-2}$ singularity and the $\left\langle\left|\varepsilon_{2}\right|^{2}\right\rangle_{0}$ term, which requires its prefactor to match the one in $\mathcal{L}_{\text {gauge }}^{\text {eff }}$, changing one but not the other results in qualitatively different long-distance behavior [cf. Eq. (88), where we discarded oscillatory terms and hence found Eq. (D21) with $\gamma=1$ ]. The presence or absence of the oscillatory terms in the total gauge-field action $\mathcal{L}_{\text {gauge }}+\mathcal{L}_{\text {ind }}$ changes both of these factors equally and, therefore, does not qualitatively affect the monopole-monopole correlation function.

[1] P. A. Lee, An end to the drought of quantum spin liquids, Science 321, 1306 (2008).

[2] L. Balents, Spin liquids in frustrated magnets, Nature (London) 464, 199 (2010).

[3] L. Savary and L. Balents, Quantum spin liquids: A review, Rep. Prog. Phys. 80, 016502 (2016).

[4] Y. Zhou, K. Kanoda, and T.-K. Ng, Quantum spin liquid states, Rev. Mod. Phys. 89, 025003 (2017).
[5] C. Broholm, R. J. Cava, S. A. Kivelson, D. G. Nocera, M. R. Norman, and T. Senthil, Quantum spin liquids, Science 367, eaay0668 (2020).

[6] X.-G. Wen, Quantum orders and symmetric spin liquids, Phys. Rev. B 65, 165113 (2002).

[7] A. Hamma, R. Ionicioiu, and P. Zanardi, Bipartite entanglement and entropic boundary law in lattice spin systems, Phys. Rev. A 71, 022315 (2005). 
[8] M. Levin and X.-G. Wen, Detecting Topological Order in a Ground State Wave Function, Phys. Rev. Lett. 96, 110405 (2006).

[9] A. Kitaev and J. Preskill, Topological Entanglement Entropy, Phys. Rev. Lett. 96, 110404 (2006).

[10] A. Kitaev, Fault-tolerant quantum computation by anyons, Ann. Phys. 303, 2 (2003).

[11] A. Kitaev, Anyons in an exactly solved model and beyond, Ann. Phys. 321, 2 (2006).

[12] H. Yao and S. A. Kivelson, Exact Chiral Spin Liquid with Non-Abelian Anyons, Phys. Rev. Lett. 99, 247203 (2007).

[13] V. Chua, H. Yao, and G. A. Fiete, Exact chiral spin liquid with stable spin Fermi surface on the kagome lattice, Phys. Rev. B 83, 180412(R) (2011).

[14] R. Moessner and K. S. Raman, Quantum dimer models, in Introduction to Frustrated Magnetism: Materials, Experiments, Theory, edited by C. Lacroix, P. Mendels, and F. Mila (Springer, Berlin, 2011), p. 437.

[15] D. N. Sheng and L. Balents, Numerical Evidences of Fractionalization in an Easy-Axis Two-Spin Heisenberg Antiferromagnet, Phys. Rev. Lett. 94, 146805 (2005).

[16] C. Hickey and S. Trebst, Emergence of a field-driven U(1) spin liquid in the Kitaev honeycomb model, Nat. Commun. 10, 530 (2019).

[17] S. V. Isakov, Y. B. Kim, and A. Paramekanti, Spin-Liquid Phase in a Spin-1/2 Quantum Magnet on the Kagome Lattice, Phys. Rev. Lett. 97, 207204 (2006).

[18] L. Dang, S. Inglis, and R. G. Melko, Quantum spin liquid in a spin- $\frac{1}{2} X Y$ model with four-site exchange on the kagome lattice, Phys. Rev. B 84, 132409 (2011).

[19] Y. Kamiya, Y. Kato, J. Nasu, and Y. Motome, Magnetic three states of matter: A quantum Monte Carlo study of spin liquids, Phys. Rev. B 92, 100403(R) (2015).

[20] O. I. Motrunich, Variational study of triangular lattice spin$1 / 2$ model with ring exchanges and spin liquid state in $\kappa-(\mathrm{ET})_{2} \mathrm{Cu}_{2}(\mathrm{CN})_{3}$, Phys. Rev. B 72, 045105 (2005).

[21] D. N. Sheng, O. I. Motrunich, and M. P. A. Fisher, Spin Bosemetal phase in a spin- $\frac{1}{2}$ model with ring exchange on a two-leg triangular strip, Phys. Rev. B 79, 205112 (2009).

[22] M. S. Block, D. N. Sheng, O. I. Motrunich, and M. P. A. Fisher, Spin Bose-Metal and Valence Bond Solid Phases in a Spin1/2 Model with Ring Exchanges on A Four-Leg Triangular Ladder, Phys. Rev. Lett. 106, 157202 (2011).

[23] Y. Iqbal, F. Becca, and D. Poilblanc, Valence-bond crystal in the extended kagome spin- $\frac{1}{2}$ quantum Heisenberg antiferromagnet: A variational Monte Carlo approach, Phys. Rev. B 83, 100404(R) (2011).

[24] R. V. Mishmash, J. R. Garrison, S. Bieri, and C. Xu, Theory of a Competitive Spin Liquid State for Weak Mott Insulators on the Triangular Lattice, Phys. Rev. Lett. 111, 157203 (2013).

[25] W.-J. Hu, S.-S. Gong, and D. N. Sheng, Variational Monte Carlo study of chiral spin liquid in quantum antiferromagnet on the triangular lattice, Phys. Rev. B 94, 075131 (2016).

[26] S. Yan, D. A. Huse, and S. R. White, Spin-liquid ground state of the $S=1 / 2$ kagome Heisenberg antiferromagnet, Science 332, 1173 (2011).

[27] S. Depenbrock, I. P. McCulloch, and U. Schollwöck, Nature of the Spin-Liquid Ground State of the $S=1 / 2$ Heisenberg Model on the Kagome Lattice, Phys. Rev. Lett. 109, 067201 (2012).
[28] Y.-C. He, D. N. Sheng, and Y. Chen, Chiral Spin Liquid in a Frustrated Anisotropic Kagome Heisenberg Model, Phys. Rev. Lett. 112, 137202 (2014).

[29] S.-S. Gong, W. Zhu, and D. N. Sheng, Emergent chiral spin liquid: Fractional quantum Hall effect in a kagome Heisenberg model, Sci. Rep. 4, 6317 (2014).

[30] Z. Zhu and S. R. White, Spin liquid phase of the $S=\frac{1}{2} J_{1}-J_{2}$ Heisenberg model on the triangular lattice, Phys. Rev. B 92, 041105(R) (2015).

[31] W.-J. Hu, S.-S. Gong, W. Zhu, and D. N. Sheng, Competing spin-liquid states in the spin- $\frac{1}{2}$ Heisenberg model on the triangular lattice, Phys. Rev. B 92, 140403(R) (2015).

[32] S.-S. Gong, W. Zhu, L. Balents, and D. N. Sheng, Global phase diagram of competing ordered and quantum spin-liquid phases on the kagome lattice, Phys. Rev. B 91, 075112 (2015).

[33] Y.-C. He, M. P. Zaletel, M. Oshikawa, and F. Pollmann, Signatures of Dirac Cones in a DMRG Study of the Kagome Heisenberg Model, Phys. Rev. X 7, 031020 (2017).

[34] Y. Shimizu, K. Miyagawa, K. Kanoda, M. Maesato, and G. Saito, Spin Liquid State in An Organic Mott Insulator with a Triangular Lattice, Phys. Rev. Lett. 91, 107001 (2003).

[35] Y. Kurosaki, Y. Shimizu, K. Miyagawa, K. Kanoda, and G. Saito, Mott Transition from a Spin Liquid to a Fermi Liquid in the Spin-Frustrated Organic Conductor $\kappa-(\mathrm{ET})_{2} \mathrm{Cu}_{2}(\mathrm{CN})_{3}$, Phys. Rev. Lett. 95, 177001 (2005).

[36] S. Yamashita, Y. Nakazawa, M. Oguni, Y. Oshima, H. Nojiri, Y. Shimizu, K. Miyagawa, and K. Kanoda, Thermodynamic properties of a spin-1/2 spin-liquid state in a $\kappa$-type organic salt, Nat. Phys. 4, 459 (2008).

[37] M. Yamashita, N. Nakata, Y. Kasahara, T. Sasaki, N. Yoneyama, N. Kobayashi, S. Fujimoto, T. Shibauchi, and Y. Matsuda, Thermal-transport measurements in a quantum spin-liquid state of the frustrated triangular magnet $\kappa-(\text { BEDT-TTF })_{2} \mathrm{Cu}_{2}(\mathrm{CN})_{3}$, Nat. Phys. 5, 44 (2009).

[38] B. J. Powell and R. H. McKenzie, Quantum frustration in organic Mott insulators: from spin liquids to unconventional superconductors, Rep. Prog. Phys. 74, 056501 (2011).

[39] M. Yamashita, N. Nakata, Y. Senshu, M. Nagata, M. Yamamoto, H. R. Kato, T. Shibauchi, and Y. Matsuda, Highly mobile gapless excitations in a two-dimensional candidate quantum spin liquid, Science 328, 1246 (2010).

[40] S. Yamashita, T. Yamamoto, Y. Nakazawa, M. Tamura, and R. Kato, Gapless spin liquid of an organic triangular compound evidenced by thermodynamic measurements, Nat. Commun. 2, 275 (2011).

[41] R. Kato, Development of $\pi$-electron systems based on $\left[\mathrm{M}(\mathrm{dmit})_{2}\right](\mathrm{M}=\mathrm{Ni}$ and $\mathrm{Pd}$; dmit: 1,3-dithiole-2-thione4,5-dithiolate) anion radicals, Bull. Chem. Soc. Jpn. 87, 355 (2014).

[42] J. S. Helton, K. Matan, M. P. Shores, E. A. Nytko, B. M. Bartlett, Y. Yoshida, Y. Takano, A. Suslov, Y. Qiu, J.-H. Chung, D. G. Nocera, and Y. S. Lee, Spin Dynamics of the Spin-1/2 Kagome Lattice Antiferromagnet $\mathrm{ZnCu}_{3}(\mathrm{OH})_{6} \mathrm{Cl}_{2}$, Phys. Rev. Lett. 98, 107204 (2007).

[43] T.-H. Han, J. S. Helton, S. Chu, D. G. Nocera, J. A. RodriguezRivera, C. Broholm, and Y. S. Lee, Fractionalized excitations in the spin-liquid state of a kagome-lattice antiferromagnet, Nature (London) 492, 406 (2012). 
[44] M. R. Norman, Colloquium: Herbertsmithite and the search for the quantum spin liquid, Rev. Mod. Phys. 88, 041002 (2016).

[45] Y. Kasahara, K. Sugii, T. Ohnishi, M. Shimozawa, M. Yamashita, N. Kurita, H. Tanaka, J. Nasu, Y. Motome, T. Shibauchi, and Y. Matsuda, Unusual Thermal Hall Effect in a Kitaev Spin Liquid Candidate $\alpha-\mathrm{RuCl}_{3}$, Phys. Rev. Lett. 120, 217205 (2018).

[46] H. Takagi, T. Takayama, G. Jackeli, G. Khaliullin, and S. E. Nagler, Concept and realization of Kitaev quantum spin liquids, Nat. Rev. Phys. 1, 264 (2019).

[47] X.-G. Wen, Quantum Field Theory of Many-Body Systems, Oxford Graduate Texts (Oxford University Press, Oxford, 2004).

[48] P. Coleman, Introduction to Many-Body Physics (Cambridge University Press, Cambridge, 2015).

[49] C. Gros, Physics of projected wavefunctions, Ann. Phys. 189, 53 (1989).

[50] C. L. Kane, R. Mukhopadhyay, and T. C. Lubensky, Fractional Quantum Hall Effect in an Array of Quantum Wires, Phys. Rev. Lett. 88, 036401 (2002).

[51] J. C. Y. Teo and C. L. Kane, From Luttinger liquid to nonAbelian quantum Hall states, Phys. Rev. B 89, 085101 (2014).

[52] J. Klinovaja and D. Loss, Integer and fractional quantum Hall effect in a strip of stripes, Eur. Phys. J. B 87, 171 (2014).

[53] T. Meng, P. Stano, J. Klinovaja, and D. Loss, Helical nuclear spin order in a strip of stripes in the quantum Hall regime, Eur. Phys. J. B 87, 203 (2014).

[54] E. Sagi, Y. Oreg, A. Stern, and B. I. Halperin, Imprint of topological degeneracy in quasi-one-dimensional fractional quantum Hall states, Phys. Rev. B 91, 245144 (2015).

[55] Y. Fuji, Y.-C. He, S. Bhattacharjee, and F. Pollmann, Bridging coupled wires and lattice Hamiltonian for two-component bosonic quantum Hall states, Phys. Rev. B 93, 195143 (2016).

[56] C. L. Kane, A. Stern, and B. I. Halperin, Pairing in Luttinger Liquids and Quantum Hall States, Phys. Rev. X 7, 031009 (2017).

[57] Y. Fuji and A. Furusaki, Quantum Hall hierarchy from coupled wires, Phys. Rev. B 99, 035130 (2019).

[58] W. B. Fontana, P. R. S. Gomes, and C. A. Hernaski, From quantum wires to the Chern-Simons description of the fractional quantum Hall effect, Phys. Rev. B 99, 201113(R) (2019).

[59] Y. Imamura, K. Totsuka, and T. H. Hansson, From coupledwire construction of quantum Hall states to wave functions and hydrodynamics, Phys. Rev. B 100, 125148 (2019).

[60] J. Klinovaja and Y. Tserkovnyak, Quantum spin Hall effect in strip of stripes model, Phys. Rev. B 90, 115426 (2014).

[61] E. Sagi and Y. Oreg, Non-Abelian topological insulators from an array of quantum wires, Phys. Rev. B 90, 201102(R) (2014).

[62] T. Meng and E. Sela, Time reversal symmetry broken fractional topological phases at zero magnetic field, Phys. Rev. B 90, 235425 (2014).

[63] R. A. Santos, C.-W. Huang, Y. Gefen, and D. B. Gutman, Fractional topological insulators: From sliding Luttinger liquids to Chern-Simons theory, Phys. Rev. B 91, 205141 (2015).

[64] R. S. K. Mong, D. J. Clarke, J. Alicea, N. H. Lindner, P. Fendley, C. Nayak, Y. Oreg, A. Stern, E. Berg, K. Shtengel, and M. P. A. Fisher, Universal Topological Quantum Computation from a Superconductor-Abelian Quantum Hall Heterostructure, Phys. Rev. X 4, 011036 (2014).
[65] I. Seroussi, E. Berg, and Y. Oreg, Topological superconducting phases of weakly coupled quantum wires, Phys. Rev. B 89, 104523 (2014).

[66] A. Vaezi, Superconducting Analogue of the Parafermion Fractional Quantum Hall States, Phys. Rev. X 4, 031009 (2014).

[67] E. Sagi, A. Haim, E. Berg, F. von Oppen, and Y. Oreg, Fractional chiral superconductors, Phys. Rev. B 96, 235144 (2017).

[68] M. J. Park, S. Raza, M. J. Gilbert, and J. C. Y. Teo, Coupled wire models of interacting Dirac nodal superconductors, Phys. Rev. B 98, 184514 (2018).

[69] K. Laubscher, D. Loss, and J. Klinovaja, Fractional topological superconductivity and parafermion corner states, Phys. Rev. Research 1, 032017 (2019).

[70] D. F. Mross, A. Essin, and J. Alicea, Composite Dirac Liquids: Parent States for Symmetric Surface Topological Order, Phys. Rev. X 5, 011011 (2015).

[71] S. Sahoo, Z. Zhang, and J. C. Y. Teo, Coupled wire model of symmetric Majorana surfaces of topological superconductors, Phys. Rev. B 94, 165142 (2016).

[72] X.-C. Wu, C.-M. Jian, and C. Xu, Coupled-wire description of the correlated physics in twisted bilayer graphene, Phys. Rev. B 99, 161405(R) (2019).

[73] T. Meng, Fractional topological phases in three-dimensional coupled-wire systems, Phys. Rev. B 92, 115152 (2015).

[74] E. Sagi and Y. Oreg, From an array of quantum wires to threedimensional fractional topological insulators, Phys. Rev. B 92, 195137 (2015).

[75] T. Iadecola, T. Neupert, C. Chamon, and C. Mudry, Wire constructions of Abelian topological phases in three or more dimensions, Phys. Rev. B 93, 195136 (2016).

[76] E. Sagi, A. Stern, and D. F. Mross, Composite Weyl semimetal as a parent state for three-dimensional topologically ordered phases, Phys. Rev. B 98, 201111(R) (2018).

[77] T. Iadecola, T. Neupert, C. Chamon, and C. Mudry, Groundstate degeneracy of non-Abelian topological phases from coupled wires, Phys. Rev. B 99, 245138 (2019).

[78] S. Raza, A. Sirota, and J. C. Y. Teo, From Dirac Semimetals to Topological Phases in Three Dimensions: A Coupled-Wire Construction, Phys. Rev. X 9, 011039 (2019).

[79] M. M. Vazifeh, Weyl semimetal from the honeycomb array of topological insulator nanowires, Europhys. Lett. 102, 67011 (2013).

[80] T. Meng, A. G. Grushin, K. Shtengel, and J. H. Bardarson, Theory of a $3+1 \mathrm{~d}$ fractional chiral metal: Interacting variant of the Weyl semimetal, Phys. Rev. B 94, 155136 (2016).

[81] A. A. Nersesyan and A. M. Tsvelik, Spinons in more than one dimension: Resonance valence bond state stabilized by frustration, Phys. Rev. B 67, 024422 (2003).

[82] T. Meng, T. Neupert, M. Greiter, and R. Thomale, Coupledwire construction of chiral spin liquids, Phys. Rev. B 91, 241106(R) (2015).

[83] G. Gorohovsky, R. G. Pereira, and E. Sela, Chiral spin liquids in arrays of spin chains, Phys. Rev. B 91, 245139 (2015).

[84] A. A. Patel and D. Chowdhury, Two-dimensional spin liquids with $\mathbb{Z}_{2}$ topological order in an array of quantum wires, Phys. Rev. B 94, 195130 (2016).

[85] P.-H. Huang, J.-H. Chen, P. R. S. Gomes, T. Neupert, C. Chamon, and C. Mudry, Non-Abelian topological spin liquids 
from arrays of quantum wires or spin chains, Phys. Rev. B 93, 205123 (2016).

[86] P.-H. Huang, J.-H. Chen, A. E. Feiguin, C. Chamon, and C. Mudry, Coupled spin- $\frac{1}{2}$ ladders as microscopic models for non-Abelian chiral spin liquids, Phys. Rev. B 95, 144413 (2017).

[87] P. Lecheminant and A. M. Tsvelik, Lattice spin models for non-Abelian chiral spin liquids, Phys. Rev. B 95, 140406(R) (2017).

[88] J.-H. Chen, C. Mudry, C. Chamon, and A. M. Tsvelik, Model of chiral spin liquids with Abelian and non-Abelian topological phases, Phys. Rev. B 96, 224420 (2017).

[89] R. G. Pereira and S. Bieri, Gapless chiral spin liquid from coupled chains on the kagome lattice, SciPost Phys. 4, 004 (2018).

[90] J.-H. Chen, C. Mudry, C. Chamon, and A. M. Tsvelik, Model of spin liquids with and without time-reversal symmetry, Phys. Rev. B 99, 184445 (2019).

[91] Y. Oreg, E. Sela, and A. Stern, Fractional helical liquids in quantum wires, Phys. Rev. B 89, 115402 (2014).

[92] E. M. Stoudenmire, D. J. Clarke, R. S. K. Mong, and J. Alicea, Assembling Fibonacci anyons from a $\mathbb{Z}_{3}$ parafermion lattice model, Phys. Rev. B 91, 235112 (2015).

[93] Y.-M. Lu and A. Vishwanath, Theory and classification of interacting integer topological phases in two dimensions: A Chern-Simons approach, Phys. Rev. B 86, 125119 (2012).

[94] T. Neupert, C. Chamon, C. Mudry, and R. Thomale, Wire deconstructionism of two-dimensional topological phases, Phys. Rev. B 90, 205101 (2014).

[95] D. F. Mross, J. Alicea, and O. I. Motrunich, Explicit Derivation of Duality between a Free Dirac Cone and Quantum Electrodynamics in $(2+1)$ Dimensions, Phys. Rev. Lett. 117, 016802 (2016).

[96] D. F. Mross, J. Alicea, and O. I. Motrunich, Symmetry and Duality in Bosonization of Two-Dimensional Dirac Fermions, Phys. Rev. X 7, 041016 (2017).

[97] T. Meng, Coupled-wire constructions: A Luttinger liquid approach to topology, Eur. Phys. J.: Spec. Top. 229, 527 (2020).

[98] M. E Peskin, Mandelstam-'t Hooft duality in Abelian lattice models, Ann. Phys. 113, 122 (1978).

[99] C. Dasgupta and B. I. Halperin, Phase Transition in a Lattice Model of Superconductivity, Phys. Rev. Lett. 47, 1556 (1981).

[100] M. P. A. Fisher and D. H. Lee, Correspondence between twodimensional bosons and a bulk superconductor in a magnetic field, Phys. Rev. B 39, 2756 (1989).

[101] A. M. Polyakov, Gauge Fields and Strings, Contemporary Concepts in Physics (Harwood Academic, Chur, 1987).
[102] T. Senthil, D. T. Son, C. Wang, and C. Xu, Duality between $(2+1) d$ quantum critical points, Phys. Rep. 827, 1 (2019).

[103] X.-G. Wen, F. Wilczek, and A. Zee, Chiral spin states and superconductivity, Phys. Rev. B 39, 11413 (1989).

[104] L. B. Ioffe and A. I. Larkin, Gapless fermions and gauge fields in dielectrics, Phys. Rev. B 39, 8988 (1989).

[105] M. Hermele, T. Senthil, M. P. A. Fisher, P. A. Lee, N. Nagaosa, and X.-G. Wen, Stability of $U(1)$ spin liquids in two dimensions, Phys. Rev. B 70, 214437 (2004).

[106] S.-S. Lee, Stability of the U(1) spin liquid with a spinon Fermi surface in $2+1$ dimensions, Phys. Rev. B 78, 085129 (2008).

[107] T. Giamarchi and Oxford University Press, Quantum Physics in One Dimension (Clarendon, Oxford, 2004).

[108] A. O. Gogolin, A. A. Nersesyan, and A. M. Tsvelik, Bosonization and Strongly Correlated Systems (Cambridge University Press, Cambridge, 2004).

[109] T. Senthil, A. Vishwanath, L. Balents, S. Sachdev, and M. P. A. Fisher, Deconfined quantum critical points, Science 303, 1490 (2004).

[110] C. Lannert, M. P. A. Fisher, and T. Senthil, Quantum confinement transition in a d-wave superconductor, Phys. Rev. B 63, 134510 (2001).

[111] S. Sachdev and K. Park, Ground states of quantum antiferromagnets in two dimensions, Ann. Phys. 298, 58 (2002).

[112] F. Wilczek, Magnetic Flux, Angular Momentum, and Statistics, Phys. Rev. Lett. 48, 1144 (1982).

[113] E. Fradkin, Jordan-Wigner Transformation for Quantum-Spin Systems in Two Dimensions and Fractional Statistics, Phys. Rev. Lett. 63, 322 (1989).

[114] N. Seiberg, T. Senthil, C. Wang, and E. Witten, A duality web in $2+1$ dimensions and condensed matter physics, Ann. Phys. 374, 395 (2016).

[115] A. Karch and D. Tong, Particle-Vortex Duality from 3d Bosonization, Phys. Rev. X 6, 031043 (2016).

[116] J. Murugan and H. Nastase, Particle-vortex duality in topological insulators and superconductors, J. High Energy Phys. 05 (2017) 159.

[117] A. A. Nersesyan, A. O. Gogolin, and F. H. L. Eßler, Incommensurate Spin Correlations in Spin-1/2 Frustrated Two-Leg Heisenberg Ladders, Phys. Rev. Lett. 81, 910 (1998).

[118] X.-L. Qi and S.-C. Zhang, Topological insulators and superconductors, Rev. Mod. Phys. 83, 1057 (2011).

[119] M. Levin and T. Senthil, Deconfined quantum criticality and Néel order via dimer disorder, Phys. Rev. B 70, 220403(R) (2004).

[120] L. Savary and L. Balents, Coulombic Quantum Liquids in Spin-1/2 Pyrochlores, Phys. Rev. Lett. 108, 037202 (2012). 